\title{
Selection of highly-accreting quasars
}

\section{Spectral properties of Fe $\|_{\mathrm{opt}}$ emitters not belonging to extreme Population A}

\author{
N. Bon ${ }^{1}$, P. Marziani ${ }^{2}$, E. Bon ${ }^{1}$, C. A. Negrete ${ }^{3}$, D. Dultzin ${ }^{3}$, A. del Olmo ${ }^{4}$, \\ M. D’Onofrio ${ }^{5}$, and M. L. Martínez-Aldama ${ }^{6}$
}

\author{
1 Astronomical Observatory Belgrade, Volgina 7 11060, Serbia \\ e-mail: nbon@aob.bg.ac.rs \\ 2 INAF, Osservatorio Astronomico di Padova, 35122 Padova, Italy \\ 3 Instituto de Astronomía, UNAM, Mexico D.F. 04510, Mexico \\ 4 Instituto de Astrofisíca de Andalucía, IAA-CSIC, 18008 Granada, Spain \\ 5 Dipartimento di Fisica \& Astronomia "Galileo Galilei", Università di Padova, Padova, Italy \\ ${ }^{6}$ Center for Theoretical Physics, Polish Academy of Sciences, Al. Lotników 32/46, 02-668 Warsaw, Poland
}

Received 24 September 2019 / Accepted 9 January 2020

\begin{abstract}
Context. The quasar class of extreme Population A (xA) (also known as super-Eddington accreting massive black holes, SEAMBHs) has been hailed as potential distance indicators for cosmology.

Aims. The aim of this paper is to define tight criteria for their proper identification, starting from the main selection criterion $R_{\mathrm{FeII}}>1$, and to identify potential intruders that do not meet the selection criteria, which nonetheless have been selected as xA because of the coarseness of automatic searches. The inclusion of the spurious xA sources may dramatically increase the dispersion in the Hubble diagram of quasars, which were obtained from virial luminosity estimates.

Methods. We studied a sample of 32 low- $z$ quasars that were originally selected from the seventh data release of the Sloan Digital Sky Survey as xA or SEAMBHs, which have been proved to be almost certainly misclassified sources. All of them show moderate to strong Fe II emission and the large majority show strong absorption features in their spectra which are typical of fairly evolved stellar populations. We performed a simultaneous fit of a host galaxy spectrum, active galactic nucleus (AGN) continuum, FeII template, and emission lines to spectra, using the fitting technique based on ULySS, the full spectrum fitting package. We derived the main accretion parameters (i.e., luminosity, black hole mass, and Eddington ratio) and investigate the relation between host galaxy properties and AGN.

Results. For sources in our sample with spectral types that correspond to a relatively low Eddington ratio, we find an overall consistency between $\mathrm{H} \beta_{\mathrm{NC}}$, [OIII] $\lambda \lambda 4959,5007$ line shifts, and the mean stellar velocity obtained from the host galaxy fit (within $\lesssim|60| \mathrm{km} \mathrm{s}^{-1}$ ). Only one source in our sample qualifies as a xA source.

Conclusions. The correct classification of spectra that were contaminated by heavy absorption requires careful determination of the host galaxy spectrum. Contamination and misclassification are not usual in the identification of the xAs, nor at low $z$ or at high $z$. We find a high fraction of host galaxy spectrum; in half of the sample this is even higher than $40 \%$. When absorption lines are prominent, and the fraction of the host galaxy is high, host galaxy spectrum mimics FeII, which may result in a mistaken identification of FeII spectral features. We have identified several stellar absorption lines that, along with the continuum shape, may lead to an overestimate of $R_{\mathrm{FeII}}$, and therefore to the misclassification of sources as xA sources.
\end{abstract}

Key words. quasars: general - quasars: emission lines - quasars: absorption lines - cosmology: miscellaneous galaxies: stellar content - quasars: supermassive black holes

\section{Introduction}

Quasars show properties that make them potential cosmological probes: they are plentiful, very luminous, and detected at very early cosmic epochs (currently out to redshift 7). However, they have never been successfully exploited as distance indicators in the past decades. Their luminosity is spread over six orders of magnitude, making them antithetical to conventional standard candles. Attempts to provide one or more parameters that tightly correlate with luminosity have been largely unsuccessful in the past decades (i.e., the "Baldwin effect" did not live up to its cosmological expectations (e.g., Popović \& Kovačević 2011; Bian et al. 2012; Ge et al. 2016, see also Sulentic et al. 2000a for a synopsis up to 1999)). Even the next generations of supernova surveys are unlikely to overcome the redshift limit at $\sim 1.5$ (Hook
2013). At the time of writing this paper, there was no established distance indicator in the range of redshift $1.5 \lesssim z \lesssim 4$, where important information could be gained on the cosmic density of matter and on the dynamic nature of the dark energy (e.g., D'Onofrio \& Burigana 2009, and references therein).

Realistic expectations are now kindled by isolating a class of quasars with some constant property from which the quasar luminosity can be estimated independently of its redshift. For instance, the nonlinear relation between the ultraviolet (UV) and X-ray luminosity has been used to build the Hubble diagram up to a redshift of $\approx 5.5$ (Risaliti \& Lusso 2015, 2019). Other approaches have been tested as well (e.g., Watson et al. 2011, see also Czerny et al. 2018 for a recent review). A promising possibility is provided by quasars that accrete at high and possibly super-Eddington rates (Wang et al. 2014b). Physically, 
in a super-Eddington accretion regime, a geometrically and optically thick structure, known as a "thick disk", is expected to develop (Abramowicz et al. 1988). The accretion flow remains optically thick so that radiation pressure "fattens" it. When the mass accretion rate becomes super-Eddington, the emitted radiation is advected toward the black hole, so that the source luminosity only increases with the logarithm of accretion rate. In other words, the radiative efficiency of the accretion process is expected to decrease, yielding an asymptotic behavior of the luminosity as a function of the mass accretion rate (Abramowicz et al. 1988; Mineshige et al. 2000; Watarai et al. 2000; Sadowski 2011). In observational terms, the luminosity to black hole mass ratio $\left(L / M_{\mathrm{BH}} \propto L / L_{\mathrm{Edd}}\right)$ should tend toward a well-defined value. As the accretion rate increases above $\approx 0.1$, the disk may become "slim" at first and then "thick" in the supercritical regime (Abramowicz \& Straub 2014, and references therein). The resulting "thick" accretion disk is expected to emit a steep soft and hard X-ray spectrum with a hard X-ray photon index that was computed between 2 and $20 \mathrm{KeV}$ and converges toward $\Gamma_{\text {hard }} \approx 2.5$ (Wang et al. 2013). Observationally, results are less clear. There is a broad consensus that the soft X-ray slope and the index $\alpha_{\mathrm{oX}}$ depend on the Eddington ratio and that they can be steep at a high accretion rate (Boller et al. 1996; Wang et al. 1996; Sulentic et al. 2000b; Dewangan et al. 2002; Grupe et al. 2010; Bensch et al. 2015). In the hard X-ray domain, data on weaklined quasars, which are believed to all be xAs (Martínez-Aldama et al. 2018) suggest weakness, but not necessarily with a steep slope (Shemmer et al. 2010; Ni et al. 2018). This is possible since the X-ray emission is seen through a dense outflow. More powerful X-ray instrumentation than presently available is needed for the accurate derivation of the hard-X continuum shape of sources that are anyway X-ray weak compared to the general population of quasars (Brightman et al. 2019). Quasars hosting thick disks should radiate at a well-defined limit because their luminosity is expected to saturate close to the Eddington luminosity (thus the "extremely radiating quasars" attribution). This is the case even if the mass accretion rate becomes highly super-Eddington (Mineshige et al. 2000). Their physical and observational properties are only summarily known. However, our ability to distinguish sources in different accretion states has greatly improved thanks to the exploitation of an empirical correlation set, known as the "main sequence" (MS) of quasars (Boroson \& Green 1992; Sulentic et al. 2000a,b).

The MS concept originates from a principal component analysis that was carried out on the spectra of $\approx 80$ PalomarGreen (PG) quasars by Boroson \& Green (1992). These authors identified the first eigenvector dominated by an anticorrelation between the [OIII] $\lambda 5007$ peak intensity and the strength of optical Fe II emission. Along Eigenvector 1 (E1) the full width at half maximum of $\mathrm{H} \beta(F W H M(\mathrm{H} \beta))$ and $\mathrm{Fe}$ II prominence were also correlated (Fig. 9 of Boroson \& Green 1992): a sequence based on these optical parameters, which are easily measurable on single-epoch spectra of large samples of quasars, was defined.

The E1 in a parameter space of four dimensions (4DE1, Sulentic et al. 2000a,b, 2007) is especially useful in isolating different spectral types and, among them, the spectral types that may be associated with extreme phenomena. It is important to note that 4DE1 involves the optical, UV, and X-ray. Its dimensions are set by (1) $F W H M(\mathrm{H} \beta)$, (2) the ratio of the equivalent widths of Fe II emission at $4570 \AA$ and $\mathrm{H} \beta$, $R_{\mathrm{FeII}}=W(\mathrm{Fe}$ II $\lambda 4570) / W(\mathrm{H} \beta) \approx F(\mathrm{Fe}$ II $\lambda 4570) / F(\mathrm{H} \beta)$. Items (1) and (2) define what is has come to be known as the optical plane of main sequence (MS); The additional dimensions are (3) the photon index in the soft X-rays domain, $\Gamma_{\text {soft }}$, and (4) the blueshift of the high-ionization line C IV $\lambda 1549 \AA$. Sulentic et al. (2000a) proposed two main populations on the basis of the quasar systematic trends in the optical plane $(F W H M(\mathrm{H} \beta)$ vs. $\left.R_{\mathrm{FeII}}\right)$ of the $4 \mathrm{DE} 1$ parameter space: Population A (Pop. A) for quasars with $F W H M(\mathrm{H} \beta)<4000 \mathrm{~km} \mathrm{~s}^{-1}$ and Population B (Pop. B) for those with FWHM $(\mathrm{H} \beta)>4000 \mathrm{~km} \mathrm{~s}^{-1}$. The two populations are not homogenous and they show trends in spectral properties, especially within Pop. A (Sulentic et al. $2002)$. For this reason, the optical plane of E1 was divided into $\Delta F W H M(\mathrm{H} \beta)=4000 \mathrm{~km} \mathrm{~s}^{-1}$ and $\Delta R_{\mathrm{FeII}}=0.5$. This defines the $\mathrm{A} 1, \mathrm{~A} 2, \mathrm{~A} 3$, and $\mathrm{A} 4$ bins as $R_{\mathrm{FeII}}$ increases, and the $\mathrm{B} 1, \mathrm{~B} 1+$, and $\mathrm{B} 1++$ bins are defined as $F W H M(\mathrm{H} \beta)$ increases (see Fig. 1 of Sulentic et al. 2002). Similarly, B2, B2+ and so on for each interval of the second strip, with $R_{\text {FeII }}$ in the range $0.5-1$. Thus, spectra belonging to the same bin are expected to have fairly similar characteristics concerning line profiles as well as optical and UV line ratios (Sulentic et al. 2007; Zamfir et al. 2010). The MS may be driven by the Eddington ratio $L / L_{\text {Edd }}$ convolved with the effect of orientation (e.g., Boroson 2002; Ferland et al. 2009; Marziani et al. 2001; Shen \& Ho 2014; Sun \& Shen 2015), although this view is not void of challenges. Physically, quasars may be distinguished by differences in the Eddington ratio (mainly the horizontal axis for $\mathrm{A} 1, \mathrm{~A} 2, \mathrm{~A} 3$, etc.) or by orientation (mainly the vertical axis for a fixed black hole mass).

Quasars are considered to be high accretors (hereafter xA quasars, for extreme Population A quasars) by Marziani \& Sulentic (2014, hereafter MS14), if they satisfy the following selection criterion:

$R_{\mathrm{FeII}}=\frac{\operatorname{EW}(\mathrm{FeII} \lambda 4570)}{\operatorname{EW}(\mathrm{H} \beta)}>1.0$.

At low redshift, we can identify xA quasars following the method described in MS14, that is, by isolating sources that have $R_{\mathrm{FeII}} \geq 1$ and that belong to spectral types $\mathrm{A} 3$ and $\mathrm{A} 4$, or to bins B3 and B4 if $F W H M(\mathrm{H} \beta)>4000 \mathrm{~km} \mathrm{~s}^{-1}$. SuperEddington accretors can be identified from $\Gamma_{\text {soft }}$ and from the $\Gamma_{\text {hard }}(2-20 \mathrm{keV}$ ) as well (Wang et al. 2013, 2014b). This method requires deep spectral observations from space-borne instrumentation, and this cannot be applied to large samples at present. The MS of quasars offers the simplest selection criterion $R_{\text {FeII }}>1$. A similar selection criterion has been defined through the fundamental plane of accreting black holes (Du et al. 2016a), a relation between the Eddington ratio (or dimensionless accretion rate), and $R_{\mathrm{FeII}}$ and the $D$ parameter that is defined as the ratio between the FWHM and the dispersion $\sigma$ of the $\mathrm{H} \beta$ line profile $(F W H M / \sigma(\mathrm{H} \beta))(\mathrm{Du}$ et al. 2016a). The fundamental plane can be written as two linear relations between $\log \dot{\mathcal{M}}$ and $L_{\text {bol }} / L_{\text {Edd }}$ vs. $\approx \alpha+\beta \frac{F W H M}{\sigma}+\gamma R_{\mathrm{FeII}}$, where $\alpha(>0), \beta(<0), \gamma(>0)$ as reported by $\mathrm{Du}$ et al. (2016a). The values of the Eddington ratio and $\dot{\mathcal{M}}$ derived from the fundamental plane equation are large enough to qualify the xA sources, satisfying $R_{\mathrm{FeII}}>1$ as super-Eddington accreting massive black holes (SEAMBHs). The converse may not be true - since some SEAMBHs have been identified and correspond to spectral types A2 and even A1 (e.g., Mrk 110). The point is that $\mathrm{A} 1$ and $\mathrm{A} 2$ show the minimum value of $D$ as their $\mathrm{H} \beta$ most closely resembles Lorentzian functions. Whereas, in A3 and A4, a blue-shifted excess leads to an increase in $D$. In the following, we consider $R_{\text {FeII }}>1$ (or 1.2 if doubtful borderline cases have to be excluded, following Negrete et al. 2018, hereafter Paper I) as a necessary condition to consider a source $\mathrm{xA}$ or SEAMBH, with the two terms.

As mentioned, accretion theory supports the empirical finding of MS14 on xA sources. First, $L / L_{\text {Edd }} \sim O(1)$ (up to a few 
times the Eddington luminosity) is a physically motivated condition. The ability to obtain a redshift independent distance relies on one's knowledge of the $L / L_{\text {Edd }}$ with a small dispersion around a characteristic value in addition to one's the ability to estimate the black hole mass $\left(L / L_{\mathrm{Edd}} \propto L / M_{\mathrm{BH}}\right)$. The preliminary analysis carried out in the last two years (e.g. Paper I) emphasize the need to avoid the inclusion of "intruders" in the Hubble diagram that was built from $\mathrm{xA}$, since they can significantly increase the dispersion in the distance modulus.

In this paper, we take advantage of the sample of quasars in Paper I that were selected from an automatic analysis, and we focus on the sources that were affected by strong contamination of the host galaxy and that turned out not to be $\mathrm{xA}$ sources. The identification of large samples of xA sources that are needed for their cosmological exploitation is and will continue to be based on surveys collected from fixed apertures or at best, diffraction limited PSFs $\approx 0.1 \mathrm{arcsec}$, as in the case of Euclid (Euclid Red Book Editorial Team 2011). Therefore, the broad line emitting regions will be always unresolved, and contaminated by emission from regions that are more distant from the central continuum source. Specifically, a major role is played by the host galaxy stellar spectrum. We therefore devote the paper to a detailed study of the emission properties and the host spectrum of the "intruders" in order to better define exclusion criteria.

Section 2 describes the method followed for the sample selection. The merit of the sample is to provide sources that cover a relatively wide range of $R_{\mathrm{FeII}}$ with typical low-luminosity type-1 properties, for which several intriguing properties of the host galaxy and of the AGN can be measured for the same object: age and chemical compositions as well as radial velocity shifts of narrow emission lines that are associated with the AGN narrow line region (NLR). In addition, the host galaxy spectrum effect on the appearance of the AGN spectrum can be thoroughly analyzed. We then describe several approaches aimed at obtaining the spectroscopic components associated with the AGN continuum and the emission spectrum (Sect. 3). Section 4 provides measurements and results of the host spectrum, internal line shifts (analyzing the use of the [OII] $\lambda 3727$ doublet, in detail, whose rest frame wavelength is dependent on electron density), and narrow and broad emission line parameters. Section 5 discusses the results in the context of the quasar main sequence, trying to assess the main factors affecting the $M_{\mathrm{BH}}$ and $L / L_{\mathrm{Edd}}$ estimates in small samples. In Sect. 6 the main conclusions are reported and a summary of the paper is provided.

\section{Sample selection}

The quasar sample presented by Shen et al. (2011) consists of 105783 quasar spectra of the Sloan Digital Sky Survey (SDSS) data release 7 (DR7), which was vetted following several filters: $z<0.8$ to cover the range around $\mathrm{H} \beta$ and include the Fe II $\lambda 4570$ and $5260 \AA$ blends; (2) signal-to-noise ratio $(S / N)>20$. Only 2734 spectra satisfy these criteria, and the number decreased to 468 with (3) $R_{\mathrm{FeII}} \geq 1$. The $\mathrm{S} / \mathrm{N}$ and $R_{\mathrm{FeII}}$ were estimated through the automatic measurements after continuum normalization at $5100 \AA$. Then we measured the EW of FeII and $\mathrm{H} \beta$ in the ranges of 4435-4686 and 4776-4946, respectively (Boroson \& Green 1992 ) to estimate $R_{\mathrm{FeII}}$. Among the 468 sources Negrete et al. (2018) find that 134 of them have spectra that are either noisy or are of the intermediate type (Sy 1.5), that is, the emission of the broad component of $\mathrm{H} \beta$ is very weak compared to its narrow component, which is usually intense. These authors excluded them to have a final sample of 334 sources properly classified as Type 1 with $R_{\mathrm{FeII}} \gtrsim 1$. Thirty-two of the 334 sources show strong contamination from the host galaxy. It turned out that the host-galaxy contamination mimicked the Fe II emission features customarily found around $\mathrm{H} \beta$, leading to an overestimate of $R_{\mathrm{FeII}}$ from the automated measurement (see Sect. 4.3). The study of this sample (hereafter $\mathrm{HG}$ ) is presented in this paper, while the rest of the sample (which we found out is in part suited for our cosmological project) has been in an independent paper devoted to the exploitation of $\mathrm{xA}$ quasars for cosmological parameter estimates (Paper I).

Table 1 reports the identification, the redshift, the $g$ magnitude, the color index $g-r$, the specific flux at $6 \mathrm{~cm}$ in $\mathrm{mJy}$ (FIRST, White et al. 1997), and the log of the specific flux at $2500 \AA$, and the radio-loudness parameter $R=f_{v}(6 \mathrm{~cm}) /$ $f_{v}(2500 \AA)$ (Jiang et al. 2007; Kellermann et al. 1989). According to Jiang et al. (2007) radio sources are classified as radioquiet for $R \leq 10$, and radio-loud for $R>10$. Data reported in Table 1 are taken from the table of Shen et al. (2011), where radio properties are included by matching SDSS DR7 quasar with the FIRST. The radio flux densities are subject to a considerable uncertainty (a factor of $\approx 2$ from a coarse analysis on the FIRST maps): the sources are faint, the continuum is extrapolated from $20 \mathrm{~cm}$ to $6 \mathrm{~cm}$ using a power-law with an index of 0.5 , and their values are affected by reduction residuals in the maps. The radio power is actually modest; in the case of SDSS J151600.39+572415.7 at $z \approx 0.2, \log P_{v}\left[\mathrm{~W} \mathrm{~Hz}^{-1}\right] \approx 30.4$, which is typical of radio detected sources in spectral type A2 (Ganci et al. 2019) to which this source belongs. Similar considerations apply to the other two sources. On the basis of the results of Ganci et al. (2019), the three sources may not even be true radio-load (RL) sources in the sense of having a relativistic jet (Padovani 2017).

\section{Data analysis}

In Paper I we found a subsample of 32 sources with strong contamination from a host galaxy. That analysis was done using specfit (Kriss 1994). Here we perform data analysis by using a technique based on ULySS (Koleva et al. 2009a). The results were compared with two separate techniques. One based on specfit and STARLIGHT ${ }^{1}$, and another one based on DASpec ${ }^{2}$. Since we obtained fairly consistent results with the three techiques, we performed a more detailed analysis, such as Monte Carlo simulations and $\chi^{2}$ maps, only with ULySS. Therefore, all results presented in tables and on figures were obtained with $U L y S S$. The analysis was performed using $U L y S S^{3}$, a full spectrum fitting software package, which we adopted to fit Sy1 spectra with models representing a linear combination of

\footnotetext{
1 Fitting procedure with specfit was done as described in Paper I. Since we found prominent galactic absorption lines in residuals and since the $\mathrm{H} \beta$ profile appeared as double peaked in some cases, we considered an additional specfit component - the spectrum of NGC 3779, a quiescent giant elliptical galaxy with an evolved stellar population, as a reference template. As a second approach we used STARLIGHT (Cid Fernandes et al. 2005) to subtract the host galaxy contribution before running the specfit analysis.

2 Written by $\mathrm{Du}$ (priv. comm.). The code is used in e.g., Du et al. (2016a) and Zhang et al. (2019). DASpec is based on LevenbergMarquardt minimization and can perform multicomponent spectral fitting, including AGN continuum, emission lines, an FeII template, and host contribution simultaneously.

3 The ULySS full spectrum fitting package is available at: http:// ulyss . univ-lyon 1.fr/
} 
Table 1. Basic properties of the HG sample.

\begin{tabular}{|c|c|c|c|c|c|c|}
\hline $\begin{array}{l}\text { SDSS ID } \\
\text { (1) }\end{array}$ & $\begin{array}{c}z \\
(2)\end{array}$ & $\begin{array}{c}g \\
(3)\end{array}$ & $\begin{array}{l}g-r \\
(4)\end{array}$ & $\begin{array}{c}f(6 \mathrm{~cm}) \\
(5)\end{array}$ & $\begin{array}{c}\log f(2500 \AA) \\
(6)\end{array}$ & $\begin{array}{c}R \\
\text { (7) }\end{array}$ \\
\hline J003657.17-100810.6 & 0.19 & $17.84 \pm 0.02$ & $0.34 \pm 0.03$ & & -26.89 & \\
\hline J010933.91+152559.0 & 0.23 & $18.97 \pm 0.02$ & $0.44 \pm 0.04$ & & -27.24 & \\
\hline J011807.98+150512.9 & 0.32 & $19.16 \pm 0.02$ & $0.47 \pm 0.04$ & & -27.19 & \\
\hline J031715.10-073822.3 & 0.27 & $19.08 \pm 0.03$ & $0.56 \pm 0.05$ & & -26.93 & \\
\hline $\mathrm{J} 075059.82+352005.2$ & 0.41 & $19.37 \pm 0.02$ & $0.24 \pm 0.03$ & & -27.14 & \\
\hline J082205.19+584058.3 & 0.31 & $19.48 \pm 0.02$ & $0.46 \pm 0.04$ & & -27.21 & \\
\hline J082205.24+455349.1 & 0.30 & $18.38 \pm 0.02$ & $0.44 \pm 0.04$ & & -27.02 & \\
\hline J091017.07+060238.6 & 0.30 & $19.13 \pm 0.04$ & $0.68 \pm 0.05$ & & -27.06 & \\
\hline J091020.11+312417.8 & 0.26 & $18.73 \pm 0.01$ & $0.42 \pm 0.03$ & & -27.07 & \\
\hline J092620.62+101734.8 & 0.27 & $19.25 \pm 0.02$ & $0.55 \pm 0.03$ & & -27.53 & \\
\hline J094249.40+593206.4 & 0.24 & $18.88 \pm 0.02$ & $0.46 \pm 0.04$ & & -27.19 & \\
\hline J094305.88+535048.4 & 0.32 & $19.18 \pm 0.03$ & $0.48 \pm 0.05$ & & -27.16 & \\
\hline $\mathrm{J} 103021.24+170825.4$ & 0.25 & $18.63 \pm 0.02$ & $0.39 \pm 0.03$ & & -27.19 & \\
\hline $\mathrm{J} 105530.40+132117.7$ & 0.18 & $17.61 \pm 0.02$ & $0.22 \pm 0.04$ & & -26.64 & \\
\hline $\mathrm{J} 105705.40+580437.4$ & 0.14 & $17.66 \pm 0.05$ & $0.37 \pm 0.07$ & & -26.87 & \\
\hline J112930.76+431017.3 & 0.19 & $18.46 \pm 0.03$ & $0.60 \pm 0.04$ & & -27.37 & \\
\hline $\mathrm{J} 113630.11+621902.4$ & 0.21 & $18.72 \pm 0.02$ & $0.49 \pm 0.05$ & & -27.06 & \\
\hline J113651.66+445016.4 & 0.12 & $17.71 \pm 0.04$ & $0.59 \pm 0.05$ & 1.95 & -26.97 & 18.3 \\
\hline $\mathrm{J} 123431.08+515629.2$ & 0.30 & $19.05 \pm 0.03$ & $0.54 \pm 0.05$ & 1.07 & -27.42 & 27.9 \\
\hline $\mathrm{J} 124533.87+534838.3$ & 0.33 & $18.51 \pm 0.03$ & $0.36 \pm 0.05$ & & -27.08 & \\
\hline $\mathrm{J} 125219.55+182036.0$ & 0.20 & $18.98 \pm 0.02$ & $0.79 \pm 0.03$ & & -27.18 & \\
\hline $\mathrm{J} 133612.29+094746.8$ & 0.25 & $19.10 \pm 0.02$ & $0.49 \pm 0.04$ & & -27.33 & \\
\hline $\mathrm{J} 134748.06+404632.6$ & 0.27 & $19.17 \pm 0.02$ & $0.82 \pm 0.03$ & & -27.28 & \\
\hline $\mathrm{J} 134938.08+033543.8$ & 0.20 & $18.70 \pm 0.03$ & $0.53 \pm 0.05$ & & -27.16 & \\
\hline $\mathrm{J} 135008.55+233146.0$ & 0.27 & $18.14 \pm 0.02$ & $0.23 \pm 0.05$ & & -27.00 & \\
\hline $\mathrm{J} 141131.86+442001.0$ & 0.26 & $18.90 \pm 0.03$ & $0.61 \pm 0.04$ & & -27.18 & \\
\hline $\mathrm{J} 143651.50+343602.4$ & 0.30 & $19.22 \pm 0.01$ & $0.35 \pm 0.04$ & & -27.44 & \\
\hline $\mathrm{J} 151600.39+572415.7$ & 0.20 & $18.41 \pm 0.02$ & $0.58 \pm 0.04$ & 2.27 & -26.96 & 20.4 \\
\hline $\mathrm{J} 155950.79+512504.1$ & 0.24 & $18.82 \pm 0.02$ & $0.39 \pm 0.04$ & & -27.26 & \\
\hline $\mathrm{J} 161002.70+202108.5$ & 0.22 & $18.80 \pm 0.02$ & $0.61 \pm 0.03$ & & -27.25 & \\
\hline $\mathrm{J} 162612.16+143029.0$ & 0.26 & $19.71 \pm 0.02$ & $0.88 \pm 0.03$ & & -27.62 & \\
\hline $\mathrm{J} 170250.46+334409.6$ & 0.20 & $18.14 \pm 0.01$ & $0.41 \pm 0.02$ & & -27.10 & \\
\hline
\end{tabular}

Notes. (1) SDSS name, (2) redshift, (3) $g$ magnitude, (4) color index $g-r$, (5) specific flux at $6 \mathrm{~cm}$ in $\mathrm{mJy}\left(=10^{-26} \mathrm{erg} \mathrm{s}^{-1} \mathrm{~cm}^{-2} \mathrm{~Hz}^{-1}\right),(6) \log$ of the specific flux per unit frequency at $2500 \AA$, in $\mathrm{erg} \mathrm{s}^{-1} \mathrm{~cm}^{-2} \mathrm{~Hz}^{-1}$, (7) radio-loudness parameter $R \equiv f_{v}(6 \mathrm{~cm}) / f_{v}(2500 \AA)$ Jiang et al. (2007).

nonlinear model components including the following - AGN continuum, a host galaxy, an Fe II template, and emission lines. The detailed description is given in Bon et al. (2016), where ULySS was used for the first time to fit Sy 1 spectra.

Before running the fitting procedure, we converted vacuum wavelengths into air, using the IAU definition, provided by Morton (1991), since the wavelength calibration of the SDSS spectra is in a heliocentric vacuum wavelength, while the components of the model are in air wavelengths. Therefore, all analyses were done in air wavelengths.

We adjusted ULySS to analyze simultaneously all components that contribute to the flux in the wavelength region $\lambda \lambda=$ $[3700,6800] \AA$. The model that we used for the fit represents a bounded linear combination of nonlinear components including the following - (i) stellar population spectrum, which was convolved with a line-of-sight velocity broadening function; (ii) an AGN continuum model that is represented with a power law function; (iii) a sum of Gaussian functions accounting for AGN emission lines in the analyzed spectral domain, and (iv) an Fe II template.

In order to eliminate overall shape differences between the observed stellar and galactic spectra, the model is multiplied by a polynomial function that is a linear combination of Legendre polynomials. The introduction of this polynomial in the fit ensures that results are insensitive to the Galactic extinction, normalization and the flux calibration of a galaxy and stellar template spectra (Koleva et al. 2008). The polynomial replaces the prior normalization to the pseudo-continuum that other methods need. We used a third order of the polynomial in the fit in order to model at best the extinction function and, at the same time, to prevent that the higher order terms of the polynomial affect the fit of broad emission lines and AGN continuum.

The single stellar population spectra (SSP) used for the fit of the host galaxy are spline interpolated over an age-metallicity grid of stellar population models. We used the library of SSPs that were computed by Vazdekis (1999) with the Miles library (Sánchez-Blázquez et al. 2006).

Emission lines are fit with the sum of Gaussians. All Balmer lines, as well as HeII are fit with the following four components - a narrow component, two broad components that fit the wings of the lines and a very broad component. To tie widths, shifts, and intensities of the [O III] lines, we defined two separate components of the model as a narrow component and a semibroad component. The intensity ratio between [O III] $\lambda 5007$ and [O III] $\lambda 4959$ lines was kept to 3:1 (Dimitrijević et al. 2007). The rest of emission lines are mainly fit with two Gaussians for the fit of narrow and semi-broad components. Even though, in some cases, the fit was possible with a smaller number of Gaussian components, in order to stay consistent and perform the analysis in the same way for all spectra, we used the same number of components for the whole sample.

We used the semi-empirical FeII template by Marziani et al. (2009), which was obtained from a high resolution spectrum of I Zw 1 , starting from $4000 \AA$. In the range underlying the $\mathrm{H} \beta$ profile the FeII emission was modeled with the help of FeII emission from the photoionization code CLOUDY version 07.01 (Ferland et al. 1998).

The AGN model was generated with the same sampling and at the same resolution as the observations, and the fit was performed in the pixel space. The fitting method consists of a nonlinear minimization procedure for minimizing the $\chi^{2}$ between an observed spectrum and a model. The fitting procedure applies the Levenberg-Marquardt minimization technique (Marquardt 1963). The coefficients of the multiplicative polynomial are determined by the least-squares method at each evaluation of the function minimized by the Marquardt-Levenberg routine. Additionally, the weight of each component is determined using a bounding value least-square method (Lawson \& Hanson 1995) at each iteration.

The simultaneous fit of all components in the model, which also implies the simultaneous analysis of kinematic and evolutionary parameters of the stellar population, minimizes, in the most efficient way, many degeneracies between the AGN model components reported in the literature, such as: (i) the degeneracy between fractions of AGN continuum and the host galaxy (Bon et al. 2014; Moultaka 2005), (ii) SSP age-metallicity degeneracy (Koleva et al. 2009a), and (iii) degeneracy between stellar velocity dispersion and SSP metallicity (Koleva et al. 2009b).

\section{Results}

\subsection{Immediate SSP and spectral classification results}

The results of the host galaxy single population analysis with ULySS are reported in Table 2. The columns in the table are as follows: SDSS ID; the specific flux measured at $5100 \AA$ (as proposed by Richards et al. 2006); the light fraction of power law continuum; the power law spectral index; Cols. 5-8 report the following information on the SSP analysis: the light fraction of the host galaxy, the SSP age, and the SSP shift with respect to the rest frame (defined by the SDSS-provided redshift value). The shift of the $\mathrm{H} \beta$ narrow component $\left(\mathrm{H} \beta_{\mathrm{NC}}\right)$ and the 


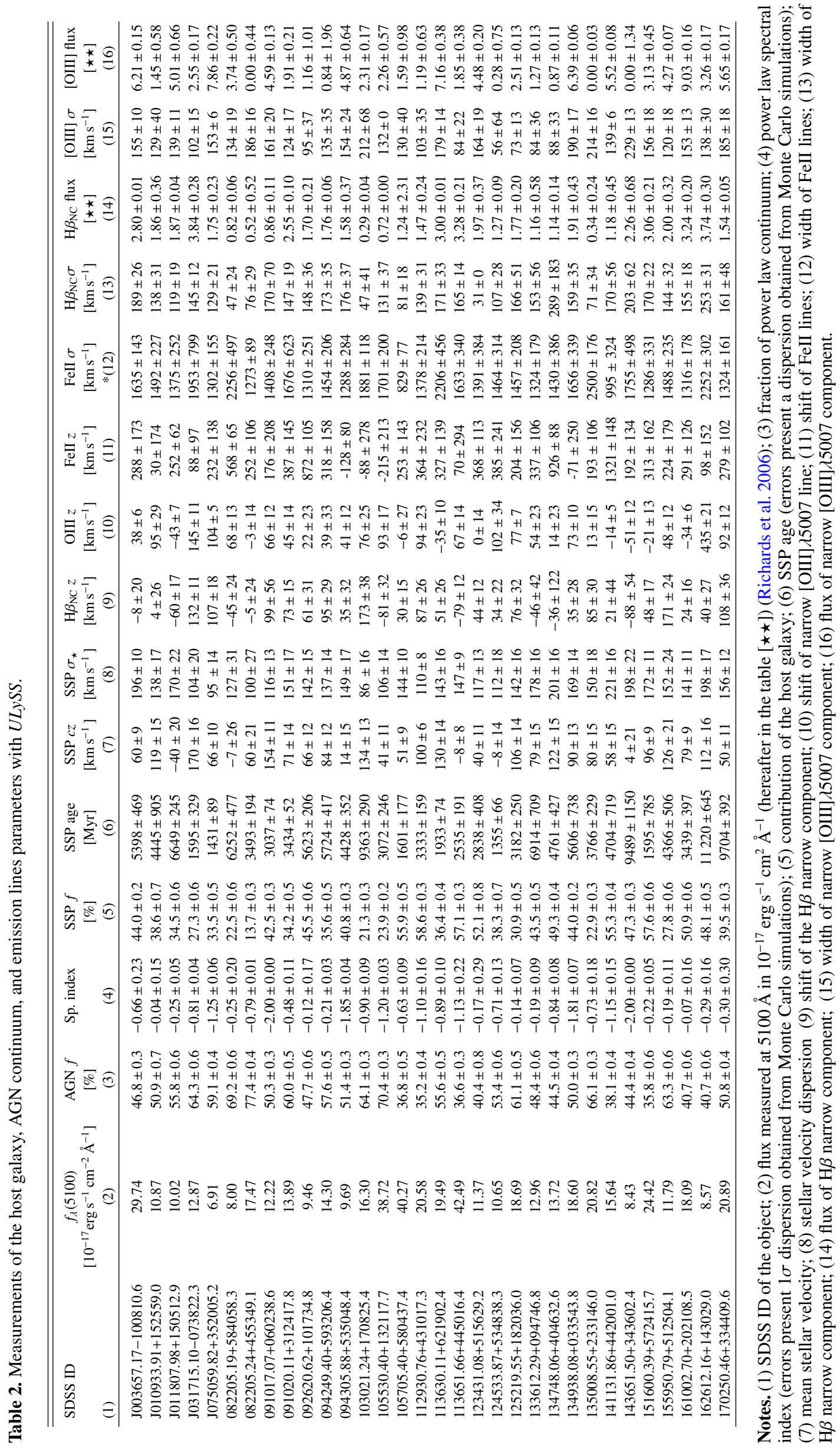


Table 3. Spectral classification.

\begin{tabular}{|c|c|c|c|c|c|c|c|c|c|c|c|c|c|}
\hline $\begin{array}{l}\text { SDSS ID } \\
\text { (1) }\end{array}$ & $\begin{array}{c}F W H M \mathrm{H} \beta_{\mathrm{BC}} \\
\text { (2) }\end{array}$ & $\begin{array}{c}\text { Flux } \mathrm{H} \beta_{\mathrm{BC}} \\
(3)\end{array}$ & $\begin{array}{c}R_{\mathrm{FeII}} \\
(4)\end{array}$ & $\begin{array}{c}E V 1_{\text {class }} \\
(5)\end{array}$ & $\begin{array}{c}\mathrm{AGN}_{\text {class }} \\
(6)\end{array}$ & $\begin{array}{c}F W H M\left(\mathrm{H} \beta_{\mathrm{BC}}\right)^{*} \\
(7)\end{array}$ & $\begin{array}{c}R_{\mathrm{FeII}}^{*} \\
(8) \\
\end{array}$ & $\begin{array}{l}\text { EV1 } 1_{\text {class }}^{*} \\
(9)\end{array}$ & $\begin{array}{c}\text { FW } 1 / 4 \\
(10)\end{array}$ & $\begin{array}{c}\text { FW 3/4 } \\
(11)\end{array}$ & $\begin{array}{c}\text { FW 9/10 } \\
(13)\end{array}$ & $\begin{array}{l}\text { C } 1 / 4 \\
(13)\end{array}$ & $\begin{array}{c}\text { C } 1 / 2 \\
(14)\end{array}$ \\
\hline J003657.17-100810.6 & 4827 & $27.1 \pm 2.3$ & $0.9 \pm 0.1$ & B2 & S1 & 5860 & 1.1 & B3 & 8634 & 3104 & 1933 & 239 & -300 \\
\hline J010933.91+152559.0 & 4903 & $36.4 \pm 6.7$ & $0.8 \pm 0.2$ & B2 & S1 & 4629 & 0.9 & B2 & 7675 & 3038 & 1795 & 526 & 166 \\
\hline J011807.98+150512.9 & 9501 & $36.7 \pm 5.6$ & $0.5 \pm 0.2$ & B2+ & S1 & 11091 & 0.9 & B2+ & 13381 & 6103 & 3675 & 1390 & 1423 \\
\hline J031715.10-073822.3 & 8564 & $28.1 \pm 1.2$ & $0.3 \pm 0.1$ & B1+ & S1 & 9024 & 0.8 & B2+ & 12605 & 4473 & 2475 & 1063 & 198 \\
\hline J075059.82+352005.2 & 6830 & $38.8 \pm 4.4$ & $0.6 \pm 0.2$ & B2 & S1 & 8008 & 0.8 & B2+ & 10371 & 5103 & 1718 & 541 & -141 \\
\hline $\mathrm{J} 082205.19+584058.3$ & 8136 & $36.7 \pm 4.1$ & $0.8 \pm 0.1$ & B2+ & - & 7729 & 0.9 & B2 & 11652 & 5171 & 3102 & -316 & -311 \\
\hline J082205.24+455349.1 & 7538 & $32.8 \pm 4.6$ & $0.9 \pm 0.1$ & B2 & $\mathrm{S} 1$ & 9677 & 1.2 & B3+ & 11626 & 5044 & 3591 & 800 & 595 \\
\hline J091017.07+060238.6 & 7042 & $32.5 \pm 4.5$ & $0.5 \pm 0.3$ & B2 & AGN & 7947 & 0.7 & B2 & 9888 & 5592 & 4695 & 562 & 93 \\
\hline J091020.11+312417.8 & 5807 & $16.6 \pm 1.2$ & $0.5 \pm 0.2$ & B1 & - & 6469 & 0.7 & B2 & 7120 & 4770 & 1170 & 447 & 474 \\
\hline J092620.62+101734.8 & 4906 & $28.4 \pm 7.0$ & $0.7 \pm 0.3$ & B2 & S1 & 8350 & 0.9 & B2+ & 8287 & 2972 & 1728 & 166 & 320 \\
\hline J094249.40+593206.4 & 3880 & $19.9 \pm 0.7$ & $1.1 \pm 0.2$ & A3 & $\mathrm{S} 1$ & 3982 & 1.2 & $\mathrm{~A} 3$ & 7252 & 2219 & 1318 & 209 & 1127 \\
\hline J094305.88+535048.4 & 9578 & $34.5 \pm 3.2$ & $0.4 \pm 0.2$ & B1+ & AGN & 9457 & 0.5 & B2+ & 13643 & 6343 & 4626 & -478 & -483 \\
\hline $\mathrm{J} 103021.24+170825.4$ & 6416 & $50.4 \pm 6.4$ & $1.2 \pm 0.2$ & B3 & - & 6285 & 1.7 & B4 & 8414 & 4275 & 2065 & -251 & -160 \\
\hline $\mathrm{J} 105530.40+132117.7$ & 5228 & $12.6 \pm 0.7$ & $1.5 \pm 0.1$ & B3 & - & 8013 & 1.8 & B4+ & 7985 & 3233 & 1926 & -791 & -1012 \\
\hline $\mathrm{J} 105705.40+580437.4$ & 2418 & $26.8 \pm 2.5$ & $0.6 \pm 0.1$ & A2 & - & 2826 & 0.8 & $\mathrm{~A} 2$ & 3868 & 1520 & 898 & 208 & 239 \\
\hline $\mathrm{J} 112930.76+431017.3$ & 5176 & $22.8 \pm 5.2$ & $0.7 \pm 0.3$ & B2 & $\mathrm{S} 1$ & 9154 & 1.1 & B3+ & 8909 & 3036 & 1794 & 197 & -32 \\
\hline $\mathrm{J} 113630.11+621902.4$ & 6982 & $31.0 \pm 2.1$ & $0.6 \pm 0.1$ & B2 & AGN & 7445 & 0.9 & B2 & 11417 & 4140 & 1929 & 757 & 447 \\
\hline $\mathrm{J} 113651.66+445016.4$ & 5118 & $16.6 \pm 2.4$ & $0.7 \pm 0.2$ & B2 & $\mathrm{S} 1$ & 7214 & 1.1 & B3 & 8099 & 3181 & 1867 & 876 & 617 \\
\hline $\mathrm{J} 123431.08+515629.2$ & 5662 & $26.0 \pm 4.9$ & $0.6 \pm 0.3$ & B2 & AGN & 8896 & 0.7 & B2+ & 9765 & 4005 & 2970 & 1019 & 129 \\
\hline $\mathrm{J} 124533.87+534838.3$ & 6484 & $33.6 \pm 7.5$ & $0.5 \pm 0.3$ & B2 & AGN & 6956 & 0.7 & B2 & 10050 & 4353 & 3043 & -802 & -207 \\
\hline $\mathrm{J} 125219.55+182036.0$ & 6272 & $29.5 \pm 2.7$ & $0.6 \pm 0.1$ & B2 & - & 7675 & 0.8 & B2 & 10749 & 3038 & 1242 & -497 & -426 \\
\hline $\mathrm{J} 133612.29+094746.8$ & 5247 & $26.5 \pm 5.0$ & $1.0 \pm 0.2$ & B2 & - & 6556 & 1.3 & B3 & 7310 & 3664 & 1248 & -255 & 80 \\
\hline $\mathrm{J} 134748.06+404632.6$ & 4427 & $29.3 \pm 3.0$ & $0.4 \pm 0.2$ & B1 & $\mathrm{S} 1$ & 5208 & 0.5 & B1 & 6638 & 2837 & 1730 & 521 & 650 \\
\hline $\mathrm{J} 134938.08+033543.8$ & 3781 & $19.1 \pm 1.1$ & $0.8 \pm 0.1$ & $\mathrm{~A} 2$ & AGN & 3464 & 1.1 & $\mathrm{~A} 3$ & 6402 & 2337 & 1375 & -784 & -1208 \\
\hline $\mathrm{J} 135008.55+233146.0$ & 10145 & $46.0 \pm 1.6$ & $0.9 \pm 0.0$ & B2+ & - & 11098 & 1.2 & B3+ & 14354 & 6556 & 4003 & -11 & -19 \\
\hline $\mathrm{J} 141131.86+442001.0$ & 6219 & $20.9 \pm 2.0$ & $0.4 \pm 0.2$ & $\mathrm{~B} 1$ & $\mathrm{~S} 1$ & 6779 & 0.8 & B2 & 7736 & 4977 & 3941 & 207 & 336 \\
\hline $\mathrm{J} 143651.50+343602.4$ & 6985 & $32.5 \pm 6.3$ & $0.5 \pm 0.2$ & B2 & $\mathrm{S} 1$ & 7102 & 0.7 & B2 & 9204 & 5533 & 4565 & 806 & 583 \\
\hline $\mathrm{J} 151600.39+572415.7$ & 3798 & $16.6 \pm 0.1$ & $0.6 \pm 0.1$ & A2 & S1 & 4444 & 0.6 & B2 & 5666 & 2555 & 1519 & 326 & 145 \\
\hline $\mathrm{J} 155950.79+512504.1$ & 4765 & $31.4 \pm 4.0$ & $0.7 \pm 0.2$ & B2 & S1 & 5593 & 0.9 & B2 & 7385 & 1790 & 964 & -29 & 165 \\
\hline $\mathrm{J} 161002.70+202108.5$ & 4424 & $19.5 \pm 1.2$ & $1.1 \pm 0.1$ & B3 & - & 5080 & 1.2 & B3 & 6428 & 2834 & 1728 & 416 & 441 \\
\hline $\mathrm{J} 162612.16+143029.0$ & 9017 & $31.8 \pm 2.6$ & $1.2 \pm 0.1$ & B3+ & - & 9620 & 1.4 & B3+ & 12665 & 5783 & 3511 & -805 & -803 \\
\hline $\mathrm{J} 170250.46+334409.6$ & 5588 & $42.7 \pm 4.6$ & $0.5 \pm 0.2$ & $\mathrm{~B} 2$ & AGN & 6528 & 0.7 & $\mathrm{~B} 2$ & 10023 & 3935 & 2902 & 444 & -137 \\
\hline
\end{tabular}

Notes. (1) SDSS ID of the object; (2) $F W H M \mathrm{H} \beta$; (3) $\mathrm{F} \mathrm{H} \beta$ BC - flux of broad $\mathrm{H} \beta$ line component; (4) $R_{\mathrm{FeII}}$; (5) classification of the spectra using EV1 diagram; (6) S1: Seyfert 1 according to the classification of Véron-Cetty \& Véron (2006), AGN: unclassified AGN; (7) FWHM of broad $\mathrm{H} \beta$ from Shen \& Ho (2014); (8) calculated $R_{\mathrm{FeII}}$ using data from Shen \& Ho (2014); (9) classification of spectra on EV1 diagram, calculated from Shen \& Ho (2014) data. (10) FW 1/4 - full width of H $\beta$ line; (11) FW3/4 of H $\beta$; (12) FW9/10 H $\beta$; (13) C 1/4 - centroid of $\mathrm{H} \beta$ line measured at $1 / 4$ of maximum intensity; (7) $\mathrm{C} 1 / 2$ - centroid of $\mathrm{H} \beta$ line measured at $1 / 2$ of maximum intensity.

narrow [OIII] $\lambda 5007$ line are reported in Cols. 9-10. The shift and width (the Gaussian dispersion $\sigma$ ) of FeII lines are listed in Cols. 11-12. Columns 13-16 list the flux and $\sigma$ for $\mathrm{H} \beta_{\mathrm{NC}}$ and [OIII] $\lambda 5007$. Figure A.1 shows a spectral atlas with the main components.

The classification concerning the spectral type assignment along the E1 MS optical diagram and the AGN classification according to Véron-Cetty \& Véron (2006) are presented in Table 3. The table lists the following: the FWHM and flux of $\mathrm{H} \beta_{\mathrm{BC}}$ (Cols. 2-3), $R_{\mathrm{FeII}}$ and the main sequence spectral type (Cols. 4-5), along with the classification of the catalog of VéronCetty \& Véron (2006). Sources for which no classification is given in the catalog are recognizable as type-1 AGN in the SDSS (from S1.0 to 1.8). However, the classification of some of them (for example J162612.16) might not have been easy when using old spectroscopic data because of the strong host contamination. Columns 7-8 list the $F W H M \mathrm{H} \beta_{\mathrm{BC}}$ and $R_{\mathrm{FeII}}$ following Shen et al. (2011). The corresponding spectral type is listed in Col. 9. The last columns report, in this order, the FW at $1 / 4,1 / 2$, $3 / 4$ and $0.9 \mathrm{H} \beta_{\mathrm{BC}}$ peak intensity, and the $\mathrm{H} \beta_{\mathrm{BC}}$ centroid at quarter and half maximum, $c\left(\frac{1}{4}\right)$ and $c\left(\frac{1}{2}\right)$. These parameters are useful in the asymmetry and the shift analysis, especially at $1 / 4$ of maximum intensity. Both $\mathrm{H} \beta$ and [OIII] $\lambda \lambda 4959,5007$ are often affected by asymmetries close to the line base. The $1 / 4$ maximum intensity provides a suitable level to detect and quantify these asymmetries.

\subsection{Spectral type classification along the quasar MS: Not $x A$ sources in almost all cases}

The HG sample sources remain, by all measurements, relatively strong Fe II emitters, with $R_{\mathrm{FeII}} \gtrsim 0.3$. Figure 1 shows the location of the 32 sources in the optical plane of the E1 MS (represented with red and blue circles). The $R_{\mathrm{FeII}}$ and $F W H M \mathrm{H} \beta$ place the sources predominantly into the $\mathrm{B} 2$ and $\mathrm{A} 2$ spectral bins; only one source can be considered as a genuine $\mathrm{xA}$ candidate.

There is good agreement between our measurements of $R_{\mathrm{FeII}}$ and those of Shen et al. (2011); as seen from the measurements reported in Table $2, R_{\text {FeII }}$ (Shen et al. 2011$) \approx(1.07 \pm$ $0.08) R_{\mathrm{FeII}}+(0.18 \pm 0.06)$, implying that the values from Shen et al. (2011) are systematically higher by $18 \%$. The reason for this disagreement could be that Shen et al. (2011) did not take into account the host galaxy contribution (Śniegowska et al. 2018). This analysis would imply that $5 / 32$ sources could be classified as xA with $R_{\mathrm{FeII}}>1$, following Shen et al. (2011). The number reduces to only 1 out of 32 if the most restrictive criterion $R_{\mathrm{FeII}} \geq 1.2$ is applied. Parameter $D=F W H M_{\mathrm{H} \beta} / \sigma_{\mathrm{H} \beta}$ distinguishes sources from the Fig. 1 into two groups. Sources with 


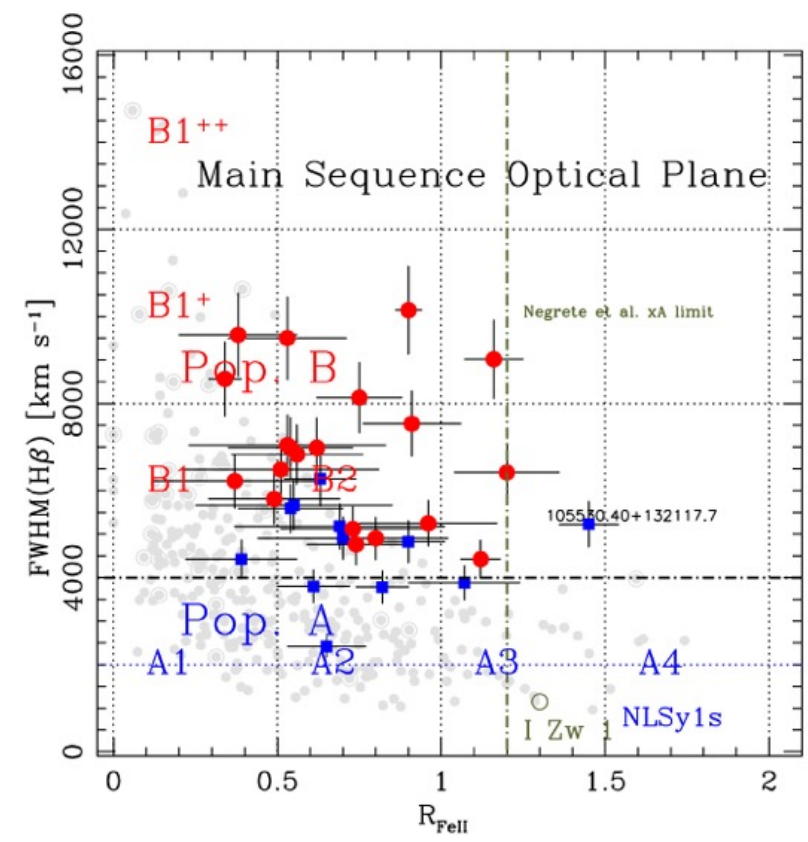

Fig. 1. Optical plane of the E1 MS, $F W H M \mathrm{H} \beta_{\mathrm{BC}}$ vs. $R_{\mathrm{FeII}}$. Sources from our sample are represented as red and blue circles; the gray symbols represent the MS from the sample of Zamfir et al. (2010), with RL sources identified by an outer circle. The red circles are sources with the $D$ parameter larger than 1.5 ; for the blue squares $D \lesssim 1.5$. The horizontal dot-dashed line marks the limit between Population A and at $F W H M=4000 \mathrm{~km} \mathrm{~s}^{-1}$. Dotted lines separate spectral types and NLSy1s. The vertical dot-dashed olive line identifies the $R_{\text {FeII }}=1.2$ limit for xA "safe" identification according to Paper I. Only one source qualifies as a true $\mathrm{xA}$ candidate. The position of the prototypical $\mathrm{xA}$ source I Zw 1 is marked.

$D>1.5$ show a more Gaussian-like $\mathrm{H} \beta$ profile. Also, $D<1.5$ implies a more Lorenzian-like profile.

As mentioned above, only one source (SDSS J105530.40+ 132117.7) is confirmed as $\mathrm{xA}$ in the full $\mathrm{HG}$ sample after SSP analysis, applying the selection criterion $R_{\mathrm{FeII}} \gtrsim 1.2$. This source is discussed individually in Sect. 5. The restriction to $R_{\mathrm{FeII}} \geq 1.2$ is operational in order to avoid contamination from a fraction of borderline objects that may not be really xA. Since typical uncertainties are $\delta R_{\mathrm{FeII}} \approx 0.1$ at $1 \sigma$ confidence level, the presence of "imitators" should be reduced by $95 \%$ the number expected with the limit at $R_{\mathrm{FeII}}=1$. Therefore, source SDSS $\mathrm{J} 105530.40+132117.7$ should be considered true $\mathrm{xA}$ and analyzed as such at a confidence level $\gtrsim 4 \sigma$.

\subsection{The reason of the $x A$ misclassification: Contamination by host galaxy absorptions}

A main issue is why the HG sources were misclassified in the first place. An example of an $\mathrm{HG}$ spectrum with the various fit components is shown in Fig. 2. We notice the high contribution of the host galaxy spectrum which is a general feature of the sample. Only one source has an SSP fraction between $10 \%$ and $20 \%$ of the total flux. In all other spectra, we find a very high fraction of the SSP component: in 16 objects this is even higher than $40 \%$. The feature that can be used as an indication of strong contamination from the host galaxy is primarily the MgIb feature that is almost always observed along with $\mathrm{H} \beta$ and [OIII] $\lambda \lambda 4959,5007$. When absorption lines are prominent, and the fraction of the host galaxy is high, we detected that SSP mimics FeII, and that this may lead to the mistaken identification of FeII spectral features (see Fig. 3). As one can see in Fig. 3, the superposition of a high fraction of the SSP on the FeII template, mimics FeII emission lines. This effect is more noticeable in the case of a high $\mathrm{S} / \mathrm{N}$, as shown on the right-hand side of Fig. 3. The combined effect of the $\mathrm{G}$ band at about $4220 \AA$ and the $\mathrm{Ca}$ absorption at $4455 \AA$ creates the impression of an excess emission around $4300 \AA$, as expected from multiplets $\mathrm{m} 27$ and $\mathrm{m} 28$ (Fe II multiplet wavelengths and information on spectral terms were taken from the Moore 1945 multiplet tables). The CaI absorption apparently delimits the blue side of the $\lambda 4570$ blend, which is mostly due to the Fe II m38 and m37 lines. At the red end of the blend, the CIII $4650 \AA$, Fe $4668 \AA$, and FeI absorptions at $4600-4650 \AA$ once again to create an illusion of a bump around $\lambda 4570$. The stellar continuum remains relatively flat down to $\approx 4400-4500 \AA$, and it steepens short-ward. This behavior also contributes to the visual impression of a FeII $\lambda 4570$ emission blend.

Similar considerations apply on the red side of $\mathrm{H} \beta$ : The $\mathrm{MgIb}$ "green triplet" cuts the continuum between the line of $\mathrm{m} 42$ at the blue edge of the blend (at $5169 \AA$ ) and the shortest wavelength line of $\mathrm{m} 49$ at $5197 \AA$. The FeI absorption at $5270 \AA$ roughly corresponds to a $5295 \AA$ dip between two pairs of lines of $\mathrm{m} 48$ and $\mathrm{m} 49$ (5265 $\AA$ and $5316 \AA$, corresponding to the transitions $z^{4} D_{1 \frac{1}{2}}^{\mathrm{o}} \rightarrow a^{4} G_{2 \frac{1}{2}}$ and $z^{4} F_{4 \frac{1}{2}}^{\mathrm{o}} \rightarrow a^{4} G_{5 \frac{1}{2}}$ : Moore 1945). Again, the Fe I absorption at $5335 \AA$ finds a rough correspondence in the dip at $\approx 5349 \AA$ between two lines of $\mathrm{m} 48$ and $\mathrm{m} 49$ (at $5316 \AA$ and $5363 \AA$ of $\mathrm{m} 48$ ). Last, at the red end of the $\lambda 5130$ blend on the red side of $\mathrm{H} \beta$, the FeI triplet at $\lambda 5406$ with the possible contribution of the HeII 5412 absorptions is also conductive to the illusion of significant emission. This explains the misidentification of the $\mathrm{xA}$ sources from the automatic procedure or from a superficial inspection.

In case of a significantly lower dispersion, the strong host contamination creates the appearance of a blue Fe II emission blend at 24570 , which is much stronger than the red one at 25300. This is the case even if the $\mathrm{S} / \mathrm{N}$ is high. This phenomenon, which can be misinterpreted if the spectral coverage does not extend below $4000 \AA$ in the rest frame, might be responsible for early claims of a different blue-to-red Fe II intensity ratio. Figure 4 illustrates how a spectrum that is heavily contaminated by the host galaxy, with insufficient spectral coverage and/or dispersion, may lead to an incorrect placement of the continuum that in turns implies an anomalous ratio between the Fe II blends on the blue and red side. Independent of resolution, little can be said about the spectrum if $S / N \lesssim 10$ : The $G$ and $\mathrm{MgIb}$ bands are lost in noise if they have $W \sim 1 \AA$. If the resolution is high, noise can be reduced by filtering, but little can be done in the case of low resolution $(\ll 1000)$. Accurate Fe II measurements necessitate an $S / N \sim 30$ in the continuum and inverse spectral resolution of $R \gtrsim 1000$ in the case of significant contamination by the host galaxy spectrum.

Monte Carlo simulations (for more information on how one can use Monte Carlo simulations with ULySS see Koleva et al. (2009a)) show the independence between the prominence of Fe II and the SSP fraction, even if we may have expected to find a correlation between these two parameters (see Fig. 5, left). Cross correlation also shows a lack of dependence between these two parameters; for instance, for the case of SDSS J124533.87+534838.3, we find $r=0.13, P=7.12 \mathrm{E}-12$. Additionally, we find degeneracies between the age of the dominant stellar population on the one hand and the fraction of the Fe II template and the width of emission lines that make up 

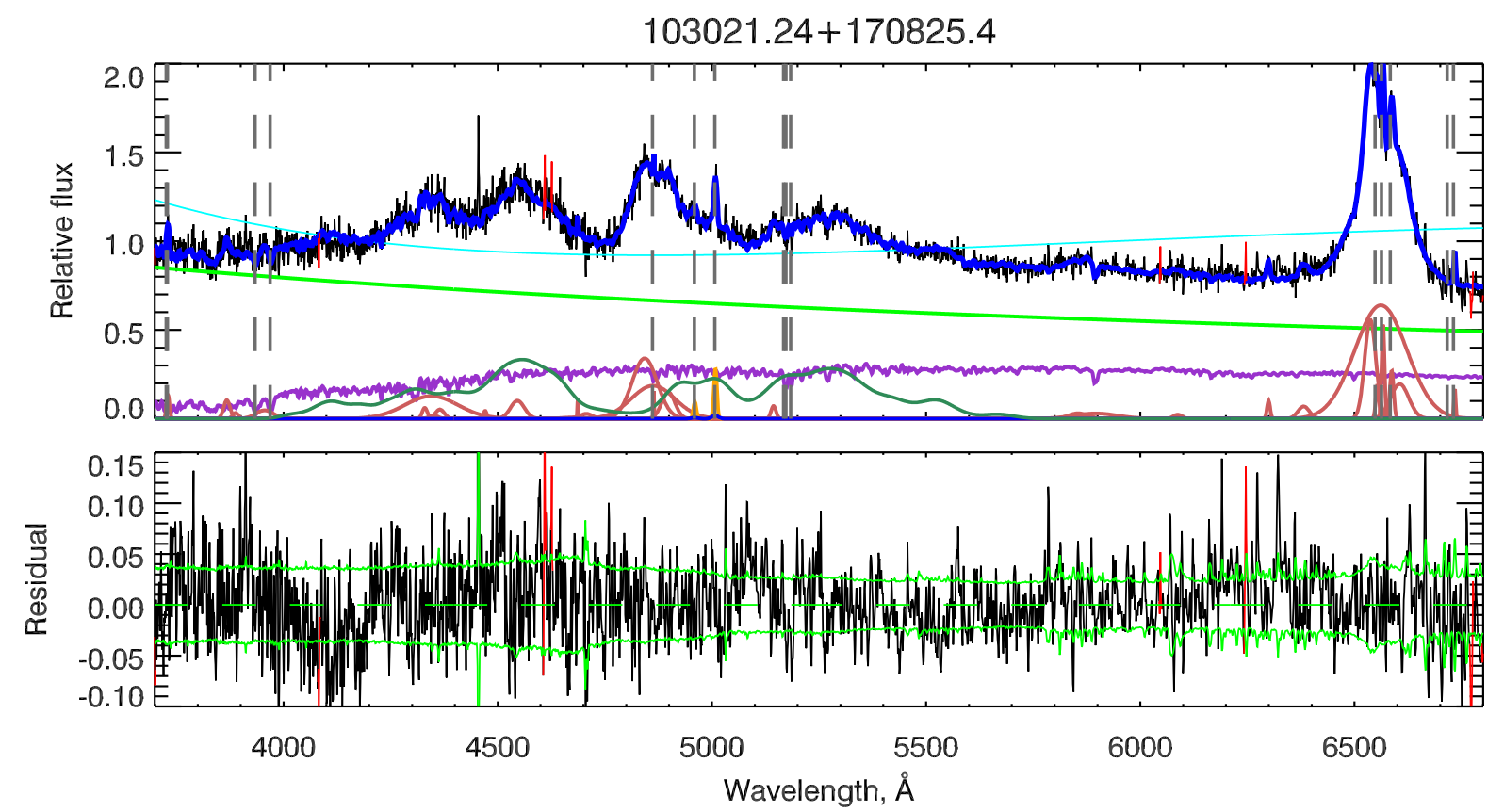

Fig. 2. Example of the spectra with strong FeII emission. In the upper panel, the black line represents the observed spectrum, the blue line represents the best fit model, and the cyan line represents the multiplicative polynomial, while the green, light red, and violet lines represent components of the ULySS best fit model as follows: violet is the stellar population, red corresponds to the emission lines, and green is the AGN continuum. Grey vertical lines mark the wavelengths in the air of the next lines as follows: [OII]3727.5, CaII H \& $\mathrm{K}, \mathrm{H} \beta$ narrow component, $[\mathrm{OIII}] \lambda \lambda 4959,5007$ narrow components and $\mathrm{MgI}$ b lines, narrow components of $\mathrm{H} \alpha$, [NII] $\lambda \lambda 46548,6583$, and [SII] $\lambda \lambda 46716,6731$, respectively. Residuals from the best fit (black line) are shown on the bottom panel. The dashed line is the zero-axis, and the green solid line shows the level of the noise. Red lines in both panels correspond to outliers of the fit.

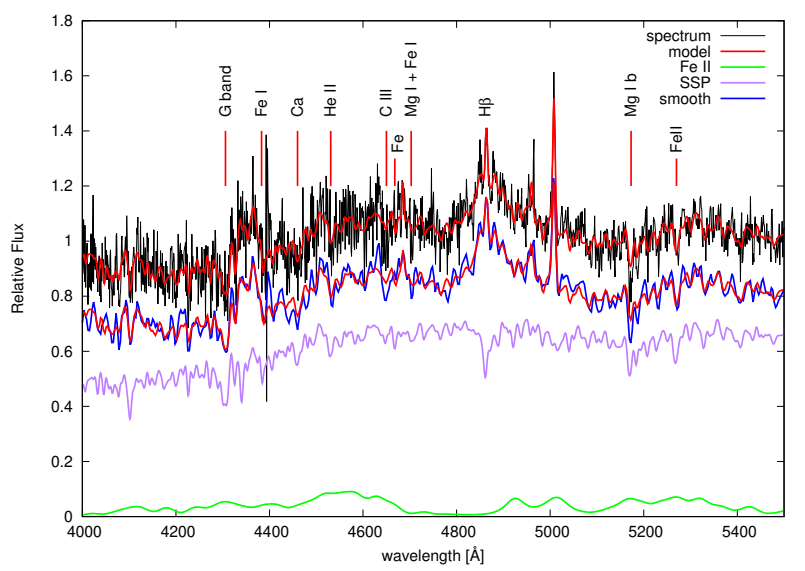

(a)

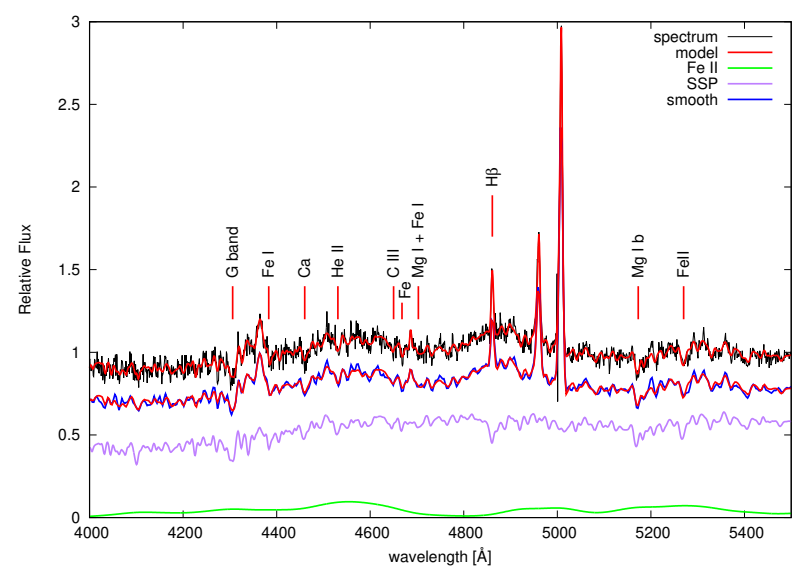

(b)

Fig. 3. Examples of spectra (J092620.62+101734.8 and J113651.66+445016.4, respectively) from our sample where the host galaxy mimics strong Fe II emission, leading to a mistaken identification of strong FeII emitters. Spectra on the plots $(a)$ and $(b)$ have a widely different S/N. The panels show as follows (from top to bottom): the real spectra and the best fitting model; the smoothed spectra overlapped with the best fitting model; single stellar population spectra that were used in the best fitting model; and the Fe II template used in the fit. Some prominent absorption lines are marked on the plots.

Fe II template, on the other hand, in the sense that we find older stellar populations when we have a lower fraction of FeII and narrower Fe II lines, with Pearson's correlation coefficient that is, for example, as large as $r=-0.87, P=8 \mathrm{E}-15$, in the case of SDSSJ $124533.87+534838$.3. Figure 5 only shows one example; different ages are involved with different objects. Relative age inferences should not be affected, although the simulations show that the actual uncertainty is larger ( $\sim$ Gyr) than the ones reported in Table 2, which are formal uncertainties from the MC simulations. Degeneracies are not necessarily due to a physical reason, and they could be due to the technical fitting problem. In order to decrease degeneracies between parameters of a stellar population and an FeII template to the minimum, it is advisable to perform the simultaneous fit of these components of the model, as we have done. This implies that the degeneracies could be even higher in the nonsimultaneous fit of the components.

\subsection{Consistency between AGN emission and host galaxy absorption spectrum}

Generally speaking, there is a good consistency between the estimators of the systemic redshift of the host galaxy and 


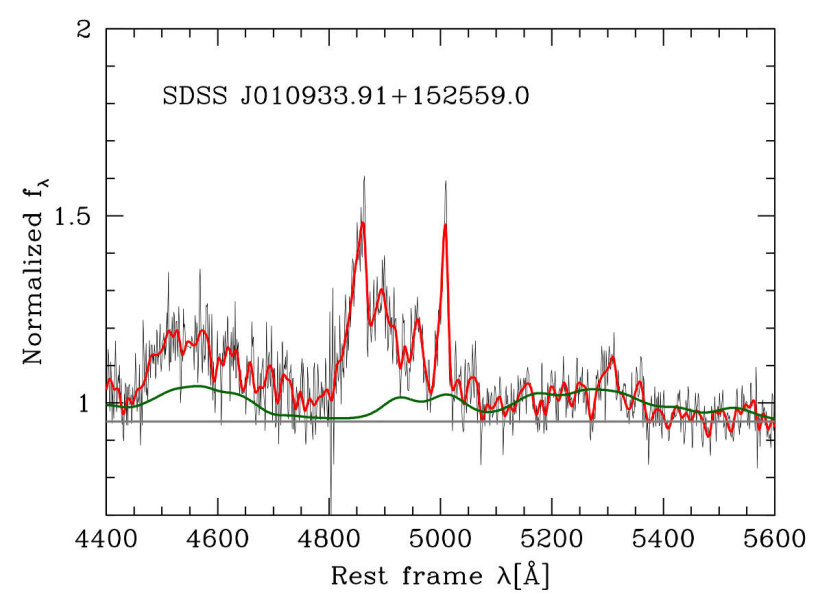

Fig. 4. Example of rest-frame spectrum whose features are misinterpreted because of heavy contamination by the absorption spectrum of the host galaxy and because of insufficient spectral coverage. The spectrum is shown with the original SDSS resolution (thin black line) and after rebinning to model data with significantly lower resolution (thick red line). The flat continuum (gray line) erroneously suggests a significant deviation from the Fe II template (dark green line).

low-ionization narrow emission lines (a fact known since the early study of Condon et al. 1985). The systemic redshift of the host may be estimated by using the atomic $21 \mathrm{~cm}$ hydrogen lines or emission from molecular $\mathrm{CO}$, which usually give results that are in close agreement (Mirabel \& Sanders 1988). A third method is provided by the absorption features of the old stellar population of the host galaxies. The tips of the narrow emission line $\mathrm{H} \beta$ and $\mathrm{H} \alpha$ can be considered as the best estimator of the systemic redshift of the host galaxy (Letawe et al. 2007). Significant differences are mostly found for the high-ionization lines such as [OIII] $\lambda \lambda 4959,5007$. The agreement between narrow low-ionization lines and the systemic redshift estimators has the important implication that any shift with respect to them can also be considered as a shift with respect to the host. This is an advantage since the inter-line shifts between low and high ionization narrow lines are easy to measure. The amplitude of the relative shifts is known to depend on the location along the main sequence. In extreme Pop. B objects shifts between Balmer lines and [OIII] $\lambda 5007$ are generally modest and $\lesssim 100 \mathrm{~km} \mathrm{~s}^{-1}$ (Eracleous \& Halpern 2004). In Pop. B [OIII] $\lambda \lambda 4959,5007$ are often blueward asymmetric and close to the line base, but the peak shift is roughly consistent with systemic redshift (see the diagram of average [OIII] $\lambda 5007$ shift along the MS in Marziani et al. 2018). In Pop. A and especially among xAs the [OIII] $\lambda 5007$ shifts become larger, and may reach several hundred $\mathrm{km} \mathrm{s}^{-1}$ in the case of the so-called blue outliers (Zamanov et al. 2002; Komossa et al. 2008; Zhang et al. 2011; Cracco et al. 2016; Marziani et al. 2016), which are believed to be relatively frequent at a high Eddington ratio or high luminosity.

Figure 6 shows the radial velocity difference between $\mathrm{H} \beta_{\mathrm{NC}}$ (gray) and [OIII] $\lambda \lambda 4959,5007$ (black) with respect to the mean stellar velocity reference frame (HG). The comparison shows that both $\mathrm{H} \beta$ and [OIII] $\lambda \lambda 4959,5007$ shifts are consistent with $\mathrm{HG}$ with some scatter $\left(54 \mathrm{~km} \mathrm{~s}^{-1}\right.$ for the case of $\mathrm{H} \beta$ and $61 \mathrm{~km} \mathrm{~s}^{-1}$ for [OIII]).

The Pearson's cross-correlation analysis between parameters yielded a high cross-correlation coefficient $(r=0.62$, $p$-value $=4.83 \mathrm{E}-05)$ between the shift of the narrow component of the $\mathrm{H} \beta$ line and $c z$. On the other hand we do not find a correlation between the SSP $c z$ and the shift of a narrow component of [OIII]4959,5007 lines (Pearson's cross-correlation coefficient is just $r=0.27$, and the $p$-value $=0.12$ ).

In Fig. 7 we compare measurements of shifts, which were derived from narrow components of the following different lines: $\mathrm{H} \beta$, [OIII] $\lambda 5007$, [SII] 66731 , and [OII] $\lambda 3727.5$, in respect to the mean stellar velocity $(c z)$. We notice small systematic effects of blueshift of the NLR with respect to the host $\left(-34 \pm 54 \mathrm{~km} \mathrm{~s}^{-1}\right.$, $-31 \pm 61 \mathrm{~km} \mathrm{~s}^{-1},-22 \pm 72$, and $-25 \pm 50$, for $\mathrm{H} \beta$, [OIII] $\lambda 5007$, [SII] 66731 and [OII] $] 3727.5$, respectively).

\section{5. [0॥] $] 33727$}

The [OII] $\lambda 3727$ doublet deserves special attention. The ratio of the two components of the doublet $\mathcal{R}=I\left({ }^{2} \mathrm{D}_{5 / 2} \rightarrow{ }^{4} \mathrm{~S}_{\frac{3}{2}}\right) /$ $I\left({ }^{2} \mathrm{D}_{3 / 2} \rightarrow{ }^{4} \mathrm{~S}_{\frac{3}{2}}\right)=I(\lambda 3729) / I(\lambda 3727)$ is sensitive to electron density $n_{\mathrm{e}}$ (Osterbrock \& Ferland 2006) with an extremely weak dependence on electron temperature (Canto et al. 1980). The laboratory wavelengths are 3726.04 and $3728.80 \AA$ in air and 3727.10 and $3729.86 \AA$ in vacuum. When the doublet is resolved, the measurement of the two component is straightforward. However, the spectral resolution of the SDSS and the intrinsic width of the [OII] $\lambda 3727$ doublet in AGNs make the doublet most often unresolved. In this case, the peak wavelength of the [OII] $\lambda 3727$ doublet is sensitive to the ratio and hence to $n_{\mathrm{e}}$ (see Appendix B for a discussion on the issue).

Since $[\mathrm{OII}] \lambda \lambda 3726,3729$ lines in our sample are not resolved, we fit the [OII] $\lambda 3727$ doublet with a single Gaussian. We used the ratio between [SII] $\lambda \lambda 6717,6731$ lines to test the correspondence between the wavelength peak and an independent density estimator (the procedure works relatively well for HII spectra, as described in the Appendix B). Only in the case of 16 objects we did succeed to fit the [SII] $\lambda \lambda 6717,6731$ lines, and therefore to calculate their intensity ratio. Table 4 lists the measured effective wavelength of the [OII] $\lambda \lambda 3726,3729$ doublet, the effective [OII] wavelength corrected for the SSP shift, and the ratio between the intensities of [SII] lines. Figure 8 represents the $R[\mathrm{SII}]=[\mathrm{SII}] \lambda 6717 / \lambda 6731$ as a function of the [OII] $\lambda \lambda 3726,3729$ doublet effective wavelength for unresolved doublets, which were corrected for the shift of SSP $c z$. We emphasize the importance of de-redshifting the spectra for SSP $c z$, since only after deshifting the spectra for SSP $c z$, the correlation between the effective wavelength of [OII] and the [SII] $\lambda \lambda 6717,6731$ intensity comes into agreement with theoretical predictions. There is an overall consistency between the prediction of the $R[\mathrm{SII}]$ and the effective wavelength $\lambda_{\mathrm{eff}}$ of the [OII] $\lambda 3727$ doublet. Only three sources deviate from a clear trend; in one case $R[\mathrm{SII}]$ suggests low density and $\lambda_{\text {eff }}$ indicates high density, while in two cases the $\lambda_{\text {eff }}$ around $3728 \AA$ in air suggests low density and $R[\mathrm{SII}]$ indicates high density. Accepted at face value, the first condition may be associated with [OII] $\lambda 3727$ being predominantly emitted in the AGN narrow-line region, while the second case may imply dominance by HII regions in the $[\mathrm{OII}] \lambda 3727$ and perhaps by a denser shock-heated region for the [SII] $\lambda \lambda 6731,6717$ emission. However, these inferences remain highly speculative, given the possibility that blueshifted emission associated with wind may contaminate the [OII] $\lambda 3727$ profile at a low-level (Kauffmann \& Maraston 2019). A larger sample with a higher $\mathrm{S} / \mathrm{N}$ is needed to ascertain whether these discrepancies are seen statistically. This may hint at a particular physical scenario.

Great care should be used in assuming a reference wavelength for [OII] 33727 . HII regions may be dominated by relatively-low density emission, yielding $\mathcal{R}[\mathrm{SII}] \approx 1.5$. On the contrary, emission within the NLR may be weighted in favor 

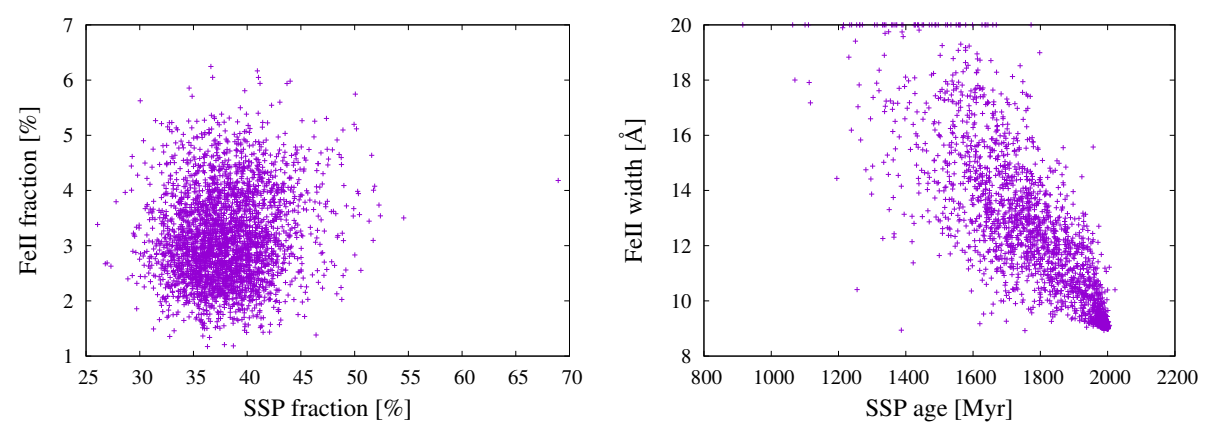

Fig. 5. Results of 3000 Monte Carlo simulations for the object SDSS J124533.87+ 534838.3 reveal no dependency the between SSP and FeII fraction (left), and they show degeneracy between the SSP age and Fe II width (right).

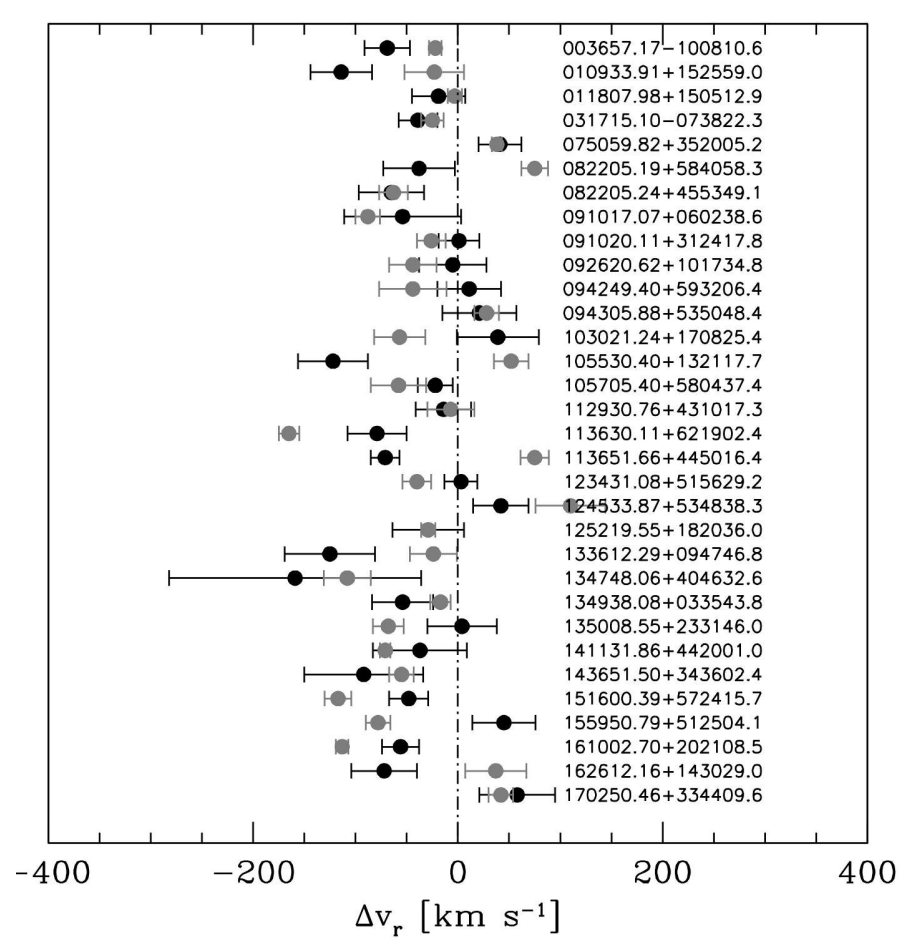

Fig. 6. Radial velocity difference between $\mathrm{H} \beta_{\mathrm{NC}}$ (gray) and [OIII] $\lambda \lambda 4959,5007$ (black) with respect to the HG reference frame.

of much higher density gas $\left(n_{\mathrm{e}} \gtrsim 10^{3} \mathrm{~cm}^{-3}\right)$, which implies $R \approx$ 0.4 . It cannot be given for granted that the spectra of our sample are dominated by NLR emission. The [OII] $\lambda 3727 /[\mathrm{OIII}] \lambda 5007$ ratio is larger in HII regions than in AGN. The SDSS aperture at the typical $z \approx 0.25$, the scale is $3.943 \mathrm{kpc} \operatorname{arcsec}^{-1}$; within the 3 arcsec aperture of $\lesssim 12 \mathrm{kpc}$, most of the light of the host galaxy should also be included. AGN show complex density behavior in their circumnuclear regions depending on the presence of nuclear outflows (Maddox 2018; Kakkad et al. 2018) and on some mixing between high-ionization narrow line region gas and low-ionization HII regions that is found for fixed size apertures (Thomas et al. 2018). Electron density is also dependent on the star formation rate (Kaasinen et al. 2017). Therefore, we might expect a dependence on the physical condition as well as the aperture size.

The dependence on $n_{\mathrm{e}}$ implies a wavelength shift that is $\lesssim 1.5 \AA$ (Appendix B) and, therefore, much larger than the accuracy of the wavelength scale of SDSS spectra. One should never forget that neglecting the dependence on density, and using a fixed wavelength as a reference, may bias redshift estimates and at least introduce a significant scatter if $\mathrm{H} \beta_{\mathrm{NC}}$ and [OII] $\lambda 3727$ redshifts are averaged together. This is the case even if it is often not possible to do otherwise. The average wavelength of the present sample is $\bar{\lambda}_{\text {eff }} \approx 3728.3 \AA$ (vacuum) and 3727.2 (air), which corresponds to $R[\mathrm{OII}]$ around unity, and $n_{\mathrm{e}} \sim 10^{2.7} \mathrm{~cm}^{-3}$ (Fig. 8.6 of Pradhan \& Nahar 2015). The value is not far from the expectation for the lower density limit typical of the NLR (Netzer 1990). These results may be a direct consequence of the location of the sources along the MS. For xA sources, $n_{\mathrm{e}}$ might be higher, thus reflecting a compact NLR with a larger density (Zamanov et al. 2002). On the other hand, if the aperture is large enough, circumnuclear and nuclear star formation may dominate the [OII] $\lambda 3727$ emission. Ascertaining the systematic trends of $\mathcal{R}$ would require extensive work; the scope of which is beyond that of the present work.

\subsection{Relation between velocity dispersion of stellar and narrow-line components}

There is considerable interest in the correlation between the masses $\left(M_{\mathrm{BH}}\right)$ of supermassive black holes (SMBHs) and the stellar velocity dispersion of the host galaxy bulge (Gebhardt et al. 2000; Ferrarese \& Merritt 2000; Kormendy \& Ho 2013) because of its important implications to the coevolution of galaxies and their SMBHs. A problem affecting the definition of the $M_{\mathrm{BH}}-\sigma_{\star}$ relation for AGNs is that a strong optical continuum emission from the AGN accretion disk can make measuring $\sigma_{\star}$ difficult. Nelson \& Whittle (1996) proposed using FWHM [OIII] $\lambda 5007$ as a proxy for $\sigma_{\star} \times 2.355$ because the [OIII] $\lambda 5007$ lines are strong and easily observable. The problem is that [OIII] $\lambda \lambda 4959,5007$ often display blue asymmetries, most often explained as an outflow component (Heckman et al. 1981), which increases the scatter of the $M_{\mathrm{BH}}-\sigma_{\star}$ relation. Measuring $\sigma_{\star}$ is more complicated in the case of AGN type 1 because of the high influence of broad emission lines and strong featureless nonstellar continuum. Furthermore, just as in several recent studies (Du et al. 2016b; Sexton et al. 2019) $\sigma_{[\mathrm{OIII}]}$ and $\sigma_{\star}$ have been measured simultaneously. Sexton et al. (2019) show that fitting the [OIII] $\lambda 5007$ line with a single Gaussian or Gauss-Hermite polynomials overestimates $\sigma_{\star}$ by more than $50 \%$. Moreover, they show that even when they exclude line asymmetries from nongravitational gas motion in a fit with two Gaussians, there is no correlation between the narrow component of $\sigma_{\text {[OIII] }}$ and $\sigma_{\star}$. The fact that these two parameters have the same range, average, and standard deviation implies that they are under the same gravitational potential (Sexton et al. 2019). They suggest that the large scatter is probably caused by the dependency between the line profiles and the light distribution and underlying kinematic field. Because of this, Sexton et al. (2019) strongly caution that the [OIII] width cannot be used as a proxy for $\sigma_{\star}$ on an individual basis. This confirms the results of Bennert et al. (2018) who show that $\sigma_{\text {[OIII] }}$ can only be used as a surrogate for $\sigma_{\star}$ in statistical studies. 


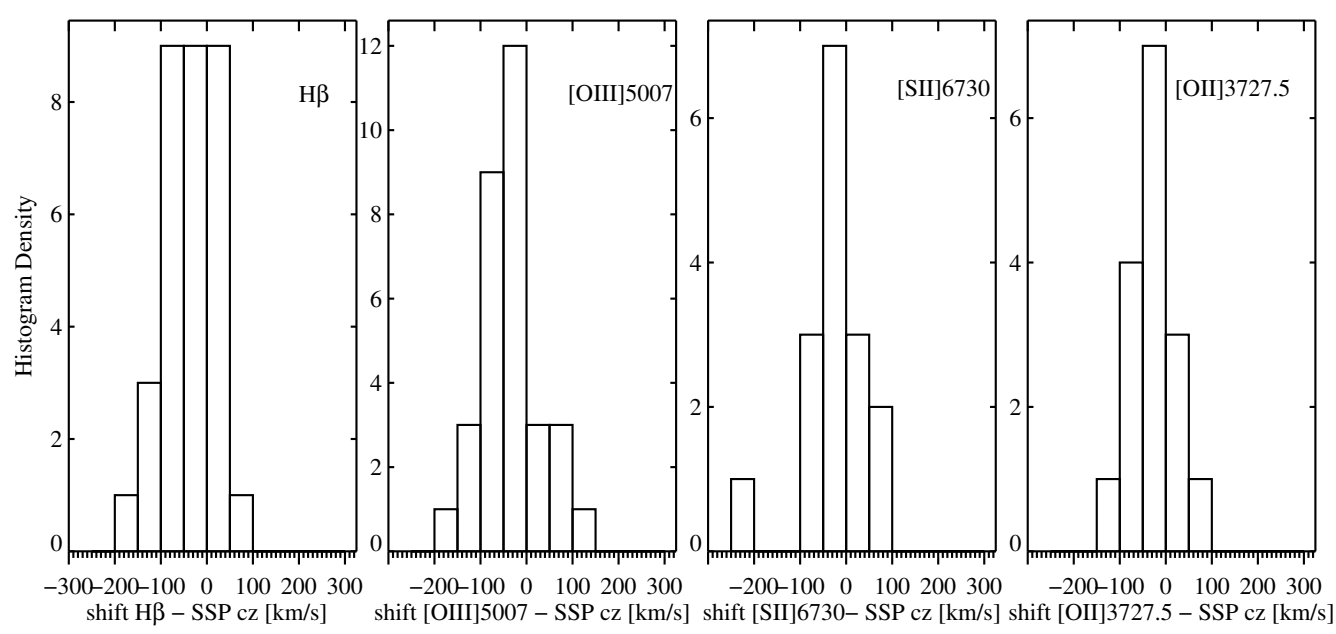

Fig. 7. Distribution of the difference in values of $z$ derived from mean stellar velocity and different emission lines. From left to right: H $\beta$, $[\mathrm{OIII}] \lambda 5007$, [SII] $] 6731$, and [OII] $] 3728.5$.

Table 4. [OII] $\lambda \lambda 3726,3729$ effective wavelengths.

\begin{tabular}{lccc}
\hline \hline SDSS ID & $\begin{array}{c}\text { [OII] } \lambda \lambda 3726,3729 \\
{[\AA]}\end{array}$ & $\begin{array}{c}{[\mathrm{OII}]^{*} \lambda \lambda 3726,3729} \\
{[\AA]}\end{array}$ & $R$ \\
$(1)$ & $(2)$ & $(3)$ & $(4)$ \\
\hline J003657.17-100810.6 & $3728.2 \pm 0.3$ & 3727.5 & $1.39 \pm 0.16$ \\
J010933.91+152559.0 & $3728.7 \pm 0.4$ & 3727.2 & $1.22 \pm 0.17$ \\
J091020.11+312417.8 & $3728.5 \pm 0.2$ & 3727.6 & $1.93 \pm 0.16$ \\
J092620.62+101734.8 & $3727.9 \pm 0.8$ & 3727.1 & $0.92 \pm 0.35$ \\
J105705.40+580437.4 & $3728.0 \pm 0.4$ & 3727.3 & $1.49 \pm 0.15$ \\
J113630.11+621902.4 & $3728.0 \pm 0.3$ & 3726.4 & $1.86 \pm 0.15$ \\
J113651.66+445016.4 & $3727.0 \pm 0.2$ & 3727.1 & $1.33 \pm 0.09$ \\
J123431.08+515629.2 & $3728.5 \pm 0.3$ & 3728.0 & $1.44 \pm 0.17$ \\
J125219.55+182036.0 & $3728.5 \pm 0.2$ & 3727.2 & $1.50 \pm 0.17$ \\
J134748.06+404632.6 & $3727.5 \pm 0.5$ & 3725.9 & $0.75 \pm 0.28$ \\
J134938.08+033543.8 & $3729.1 \pm 0.2$ & 3728.0 & $0.94 \pm 0.17$ \\
J151600.39+572415.7 & $3727.8 \pm 0.1$ & 3726.6 & $1.25 \pm 0.07$ \\
J155950.79+512504.1 & $3728.8 \pm 0.2$ & 3727.3 & $1.32 \pm 0.18$ \\
J161002.70+202108.5 & $3727.7 \pm 0.2$ & 3726.7 & $1.27 \pm 0.11$ \\
J162612.16+143029.0 & $3728.2 \pm 0.5$ & 3726.8 & $0.76 \pm 0.18$ \\
J170250.46+334409.6 & $3729.0 \pm 0.4$ & 3728.3 & $0.95 \pm 0.25$ \\
\hline
\end{tabular}

Notes. (1) SDSS ID of the object; (2) [OII] $\lambda \lambda 3726,3729$ effective wavelength; (3) [OII] $\lambda \lambda 3726,3729$ effective wavelength corrected for SSP shift; (4) $R$ - ratio between intensity of [SII] $\lambda \lambda 6717,6731$ lines.

Komossa \& Xu (2007) suggested using [SII] $\lambda \lambda 6731,6717$ as a surrogate for $\sigma_{\star}$ since the sulfur lines have a lower ionization potential and since they do not suffer from significant asymmetries. However, the scatter is comparable to that of the core of the [OIII] line.

In this work, we confirm the results of Sexton et al. (2019) since we find no correlation between $\sigma_{\star}$ and the velocity dispersion of the [OIII] $\lambda 5007$ narrow component. Instead, we find a high correlation between $\sigma_{\star}$ and the velocity dispersion of the $\mathrm{H} \beta$ narrow component $(r \approx 0.64, P \approx 1.93 \mathrm{E}-05)$. There is only an overall consistency between the values of $\sigma_{\star}$ and both $\sigma_{\text {[OIII] }}$ and $\sigma_{\mathrm{H} \beta}$. The median values of the ratios involving the three parameters and their semi inter-quartile ranges (SIQR) are as follows: $\left\langle\sigma_{[\mathrm{OIII}]} / \sigma_{\star}\right\rangle \approx 0.98 \pm 0.23$, and $\sigma_{\mathrm{H} \beta} / \sigma_{\star} \approx 1.03 \pm 0.19$, $\sigma_{[\mathrm{OIII}]} / \sigma_{\mathrm{H} \beta} \approx 0.93 \pm 0.22$.

\subsection{No strong outflows diagnosed by the [OIII] $\lambda 5007$ profile}

As mentioned above [OIII] $\lambda 5007$ lines were fit with the following two components: a narrower component that is associated

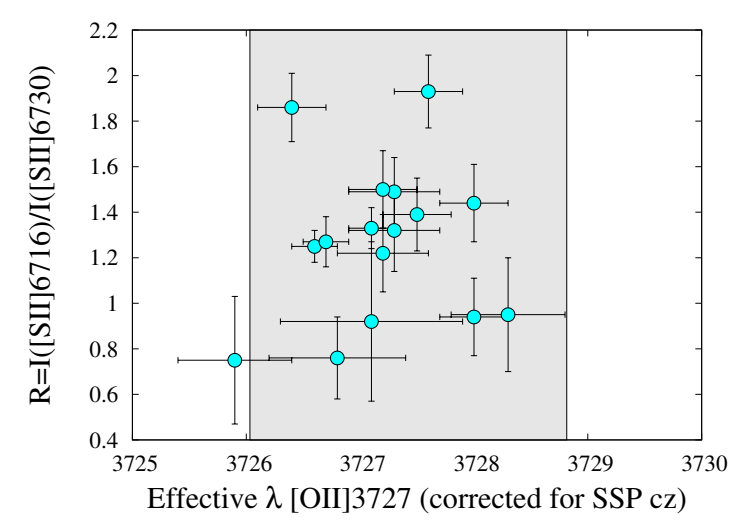

Fig. 8. Intensity ratio of $[\mathrm{SII}] \lambda 6717 / \lambda 6731$ as a function of effective wavelength in air of the unresolved [OII] $\lambda \lambda 3726,3729$ doublet corrected for SSP $c z$. Region inside the physical limits of the effective wavelengths of $[\mathrm{OII}] \lambda 3727$ is shaded on the plot.

with the core of the line, and a semi-broad component that corresponds to the radial motions (e.g., Komossa et al. 2008; Zhang et al. 2011). The spectral range around [OIII] $\lambda 5007$ lines is zoomed on the middle plot of Fig. A.1. Figure 9 shows the distribution of the shift of the semi broad component in the HG sample. As for typical type $1 \mathrm{AGN}$, the distribution of the sources in our sample is skewed to the blue, especially toward the line base. The amplitude of the blue-shifts is, however, modest and as such, it is not as strong as in the real xA sample. In looking at the full [OIII] $\lambda \lambda 4959,5007$ profiles, we see again that no object qualifies as a blue outlier following the definition of Zamanov et al. (2002). The highest amplitude blueshift at 0.9 peak intensity is $\approx-150 \mathrm{~km} \mathrm{~s}^{-1}$. The distribution of $c\left(\frac{1}{4}\right)$ values is skewed toward blueshifts, as observed in most samples (e.g., Gaur et al. 2019; Berton et al. 2016; Zhang et al. 2011), in both Pop. A and Pop. B. The conclusion is that, for most objects, we have no evidence of xA properties from the [OIII] $\lambda \lambda 4959,5007$ profiles: large shifts are common among $\mathrm{xA}$ sources, with a high frequency of blueoutliers (Paper I).

\subsection{The $H \beta$ broad profile}

We expect a significant blueward asymmetry in the $\mathrm{H} \beta$ broad profile of $\mathrm{xA}$ sources. If the profile is fit by a symmetric 

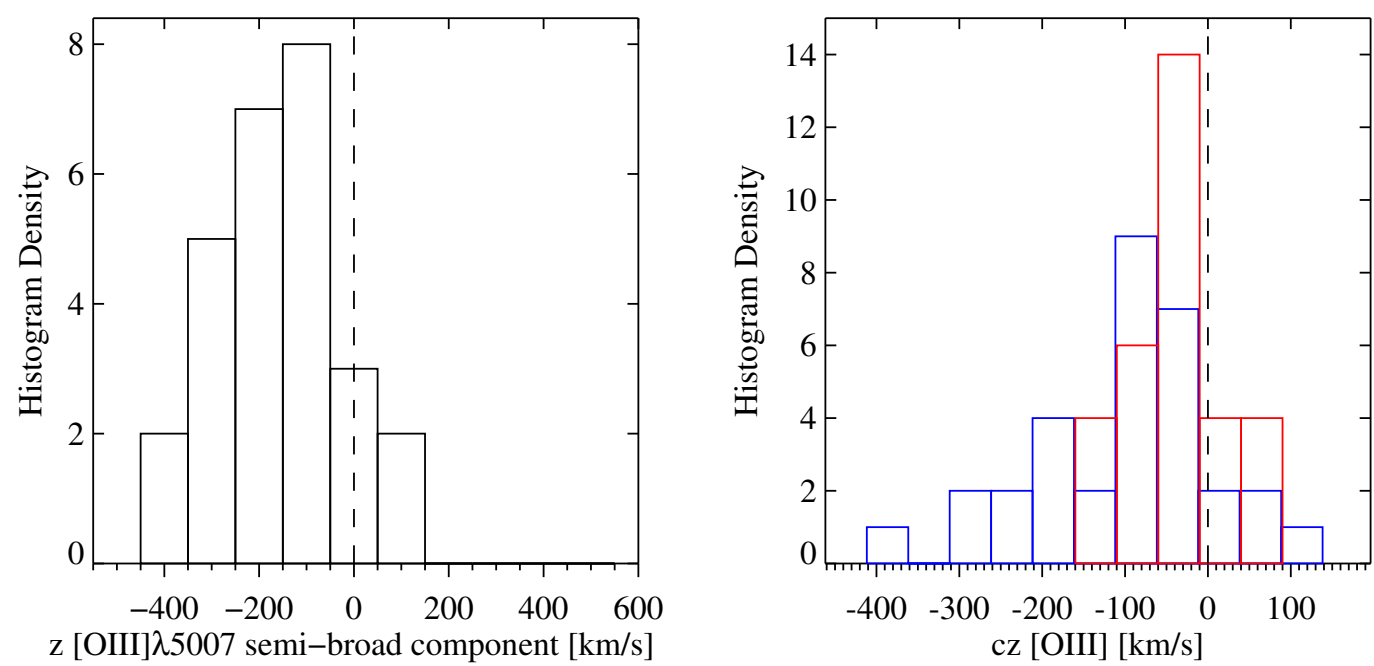

Fig. 9. Left: distribution of the shift of [OIII] $\lambda 5007$ semi-broad component. Right: distribution of the $c\left(\frac{1}{4}\right)[\mathrm{OIII}] \lambda 5007$ (blue) and of the $c(0.9)$ [OIII] $\lambda 5007$ (red).

and unshifted Lorentzian function, a residual excess emission appears on the blue side of the Lorentzian profile (several examples are shown by Negrete et al. 2018). The blueshifted emission is associated with outflows that emit more prominently in highionization lines such as CIV $\lambda 1549 \AA$ (see eg., Marziani et al. 2010 , for a systematic comparison). Table 3 reports the centroid shifts of the $\mathrm{H} \beta_{\mathrm{BC}}$ broad component. We see one clear example of blue shifted $c\left(\frac{1}{4}\right)$ in the source SDSS J105530.40+132117.7, which has the highest $R_{\mathrm{FeII}}$ in our sample. Only this object appears to be a bona fide xA source. However, sources with $c\left(\frac{1}{4}\right) \lesssim-300 \mathrm{~km} \mathrm{~s}^{-1}$ (assumed as a typical uncertainty at $1 / 4$ maximum) are rare; this is the case for only 6 out of the 33 sources. Most sources are symmetric or with the $c\left(\frac{1}{4}\right)$ displaced to the red: more than one half $(21 / 33)$ have a significant redward displacement. Prominent redward asymmetries are found among Pop. B sources, both radio quiet and radio-loud, with extreme cases in the radio-loud population (e.g., Punsly 2013). The redward excess is associated with low Eddington ratio, although its origin is still not well-understood: Tidally disrupted dusty clumps that infall toward the central black hole could be the cause of a net redshift (Wang et al. 2017), although other lines of evidence challenge this interpretation (e.g., Bon et al. 2015, and references therein).

\subsection{Properties of the host galaxy}

In almost all objects, we uncovered a very high fraction of SSP spectra out of the total flux. In the case of 17 objects the fraction is even higher than $40 \%$.

Restored mean stellar velocity $(c z)$ is between $-50 \mathrm{~km} \mathrm{~s}^{-1}$ and $170 \mathrm{~km} \mathrm{~s}^{-1}$. The stellar velocity dispersion is between $90 \mathrm{~km} \mathrm{~s}^{-1}$ and $220 \mathrm{~km} \mathrm{~s}^{-1}$. In Fig. A. 2 we show $\chi^{2}$ maps in the space of SSP mean stellar velocity $(c z)$ and SSP velocity dispersion. All SSP $c z$ that were obtained from the single best fit are in a good agreement with the values obtained from the $\chi^{2}$ maps, while SSP velocity dispersion values obtained from the single fit are usually lower then those obtained from $\chi^{2}$ maps.

We find mostly old SSP (older than 1 Gyr). The metallicities of SSPs in our 32 sample are mainly solar-like. This property is at variance with the star formation property expected for xA sources. The UV spectral properties indicate extreme metal enrichment (Martínez-Aldama et al. 2018), which is most likely associated with extreme star formation detected in the far-infrared (FIR; Sani et al. 2010; Ganci et al. 2019, in the most luminous cases, the star formation rate is SFR $\sim 10^{3} M_{\odot} \mathrm{yr}^{-1}$ ).

\section{Discussion}

\subsection{Interpretation in the Eigenvector 1 context}

We notice consistent results between our measurements and those of Shen \& Ho (2014); albeit, there is a bias in favor of a higher $R_{\text {FeII }}$ for Shen \& Ho (2014). According to the position of the spectra on the MS diagram of Fig. 1, objects are mainly Pop. B, with the exception of five sources that are of the Pop. A class. The distribution of the quasar data points is centered in Pop. B2, with 22 source objects that belong or are likely to belong to Pop. B2, including borderline objects.

Apart from the location along the MS of Fig. 1, the conclusion that the vast majority of the HG sample sources are not XAs (only one source (J105530.40) meet, in full, the criterion of Negrete et al. $\left(2018, R_{\mathrm{FeII}} \gtrsim 1.2\right)$ and qualifies as an $\mathrm{xA}$ source) is reinforced by several lines of evidence: (a) the [OIII] $\lambda \lambda 49599,5007$ profile does not have large blueshifts and [OIII] $\lambda \lambda 4959,5007$ is consistent with the rest frame; (b) the $\mathrm{H} \beta_{\mathrm{BC}}$ profile is symmetric or redward asymmetric; (c) the $\mathrm{HG}$ component is most often dominated by an old stellar population; and (d) conventional estimates of the $L / L_{\text {Edd }} \ll 1$. Regarding the last point, in Sect. 5.2 we discuss a discrepancy between $L / L_{\text {Edd }}$ estimates based on scaling laws and the new approach of the accreting black hole fundamental plane (Du et al. 2016b).

The previous analysis points toward a sample showing relatively low luminosity, and "milder" signs of nuclear activity with respect to the extreme radiators of $\mathrm{xA}$. This does not mean that a similar phenomenology concerning nuclear outflows is not occurring, but its detectability is limited to some particular manifestations, such us the blueshift of [OIII] $\lambda \lambda 44959,5007$ that is close to the line base.

\subsection{Basic physical properties}

Black hole mass estimates that use scaling laws for large samples of AGN are subject to a large uncertainty, which is due to both systematic and random errors (exhaustive reviews are given in Marziani \& Sulentic 2012; Shen 2013). However, in the case of the low- $z$ sample of the present work, we can count 
Table 5. Basic physical properties of the HG sample.

\begin{tabular}{lccccc}
\hline \hline SDSS ID & $\log L_{5100}$ & $\log M_{\mathrm{BH}}$ & $\log \frac{L}{L_{\mathrm{Edd}}}$ & $\begin{array}{c}\log M_{\mathrm{BH}} \operatorname{corr} \\
{\left[M_{\odot}\right]}\end{array}$ & $\log \frac{L}{L_{\mathrm{Edd}}} \operatorname{corr}$ \\
\hline J003657.17-100810.6 & 43.94 & 8.2 & -1.42 & 8.1 & -1.27 \\
J010933.91+152559.0 & 43.73 & 8.2 & -1.54 & 7.7 & -1.07 \\
J011807.98+150512.9 & 44.13 & 8.9 & -1.92 & 8.5 & -1.53 \\
J031715.10-073822.3 & 44.08 & 8.8 & -1.85 & 8.5 & -1.50 \\
J075059.82+352005.2 & 44.26 & 8.7 & -1.56 & 8.3 & -1.12 \\
J082205.19+584058.3 & 44.07 & 8.8 & -1.81 & 8.3 & -1.36 \\
J082205.24+455349.1 & 44.42 & 8.9 & -1.57 & 8.2 & -0.94 \\
J091017.07+060238.6 & 44.08 & 8.6 & -1.68 & 8.3 & -1.36 \\
J091020.11+312417.8 & 44.08 & 8.5 & -1.51 & 8.5 & -1.58 \\
J092620.62+101734.8 & 43.83 & 8.2 & -1.49 & 7.7 & -0.97 \\
J094249.40+593206.4 & 43.95 & 8.1 & -1.22 & 7.3 & -0.49 \\
J094305.88+535048.4 & 44.05 & 8.9 & -1.96 & 8.6 & -1.62 \\
J103021.24+170825.4 & 44.12 & 8.6 & -1.58 & 8.1 & -1.08 \\
J105530.40+132117.7 & 44.19 & 8.4 & -1.36 & 7.4 & -0.35 \\
J105705.40+580437.4 & 43.67 & 7.5 & -0.95 & 7.5 & -0.96 \\
J112930.76+431017.3 & 43.65 & 8.2 & -1.63 & 7.6 & -1.02 \\
J113630.11+621902.4 & 43.95 & 8.6 & -1.73 & 8.0 & -1.17 \\
J113651.66+445016.4 & 43.51 & 8.1 & -1.69 & 7.6 & -1.21 \\
J123431.08+515629.2 & 43.94 & 8.4 & -1.56 & 7.9 & -1.07 \\
J124533.87+534838.3 & 44.14 & 8.6 & -1.58 & 8.2 & -1.16 \\
J125219.55+182036.0 & 43.93 & 8.5 & -1.65 & 7.9 & -1.10 \\
J133612.29+094746.8 & 43.88 & 8.3 & -1.52 & 8.1 & -1.37 \\
J134748.06+404632.6 & 43.96 & 8.2 & -1.33 & 8.0 & -1.19 \\
J134938.08+033543.8 & 43.84 & 8.0 & -1.26 & 7.9 & -1.16 \\
J135008.55+233146.0 & 44.31 & 9.1 & -1.88 & 8.5 & -1.27 \\
J141131.86+442001.0 & 43.91 & 8.4 & -1.66 & 8.3 & -1.47 \\
J143651.50+343602.4 & 43.85 & 8.5 & -1.79 & 8.3 & -1.59 \\
J151600.39+572415.7 & 43.82 & 8.0 & -1.27 & 7.9 & -1.18 \\
J155950.79+512504.1 & 43.91 & 8.2 & -1.42 & 7.8 & -1.03 \\
J161002.70+202108.5 & 43.82 & 8.1 & -1.41 & 7.6 & -0.90 \\
J162612.16+143029.0 & 43.69 & 8.7 & -2.09 & 8.4 & -1.88 \\
J170250.46+334409.6 & 43.90 & 8.3 & -1.57 & 7.8 & -1.01 \\
\hline & & & & & \\
& & & & \\
\hline
\end{tabular}

on the $\mathrm{H} \beta$ line width that is considered to be a reliable "virial broadening estimator" (Trakhtenbrot \& Netzer 2012, caveats to this are found in Marziani et al. 2013a, 2019). Table 5 lists the following basic physical properties of the AGN: the log of the $5100 \AA$ AGN luminosity that was scaled by the AGN power-law continuum fraction to the total flux; the black hole mass $M_{\mathrm{BH}}$ that was computed following the prescription of Vestergaard \& Peterson (2006, hereafter VP); and $L / L_{\text {Edd }}$ (assuming the bolometric luminosity is ten times the luminosity at $5100 \AA$; Richards et al. 2006). In this table, we also present the corrected $M_{\mathrm{BH}}$ and $L / L_{\text {Edd }}$ according to the prescription of Martínez-Aldama et al. (2019) (see Sect. 5.4). The $M_{\mathrm{BH}}$ values following VP indicate a population of quasars of relatively modest $M_{\mathrm{BH}} \sim 10^{8} M_{\odot}$. Accepted at face value, $L / L_{\text {Edd }}$ is typical of Pop. B, with some objects close to the boundary between $\mathrm{A}$ and $\mathrm{B}$ but formally on the side of Pop. B, if $L / L_{\mathrm{Edd}} \approx 0.1-0.2$ is assumed as the $L / L_{\mathrm{Edd}}$ threshold for Pop. A sources.

We can write the expression of the virial mass as follows:

$M_{\mathrm{BH}}=f \frac{r_{\mathrm{BLR}}(\delta v)^{2}}{G}=f_{1}(\dot{m}, a) f_{2}(\theta \mid \dot{m}) \frac{r_{\mathrm{BLR}}(\delta v)^{2}}{G}$,

where $r_{\mathrm{BLR}}$ is the BLR radius, $a$ is the spin parameter of a black hole, and we considered the FWHM of the broad components of $\mathrm{H} \beta$ as the estimator of the virial broadening (velocity spread $\delta v=F W H M)$. We have written the structure or form factor $f$ as the product of two terms: one depending on the accretion rate and black hole spin, and one depending on orientation. The dependence of $f_{1}$ on the dimensionless accretion rate has been emphasized by the $r_{\mathrm{BLR}}$ dependence on luminosity (Du et al. 2016b), which, for xA sources, is not consistent with the general AGN population. The dependence of $f_{1}$ on the spin parameter is unknown, but it is expected since the spin influences the temperature of the accretion disk and hence the SED of the ionizing continuum (e.g., Wang et al. 2014b). To complicate the issue, the orientation effects are also expected to be dependent on $\dot{m}$ (Wang et al. 2014c), as a geometrically thin optically thick disk may be considered as a Lambertian radiator (with some limb-darkening effects at high inclination Netzer 2013); however, the orientation effects are free of the self-shadowing effects expected for a geometrically thick disk. Keeping for the moment with the simplest approach, we can compute the $M_{\mathrm{BH}}$ by using the Bentz et al. (2013, hereafter B13) correlation between $r_{\text {BLR }}$ and the optical luminosity, assuming $f=1$. The results are tightly correlated with the mass estimate obtained from the VP relation (Fig. 10). The extremely tight correlation is expected as the VP assumes the same virial relation and only a slightly different value of the zero point and of the $r_{\mathrm{BLR}}-L$ correlation. The small bias between the two relations is understood in terms of a constant difference in the $f$ factor since the VP assumed $f=0.75$. In both 

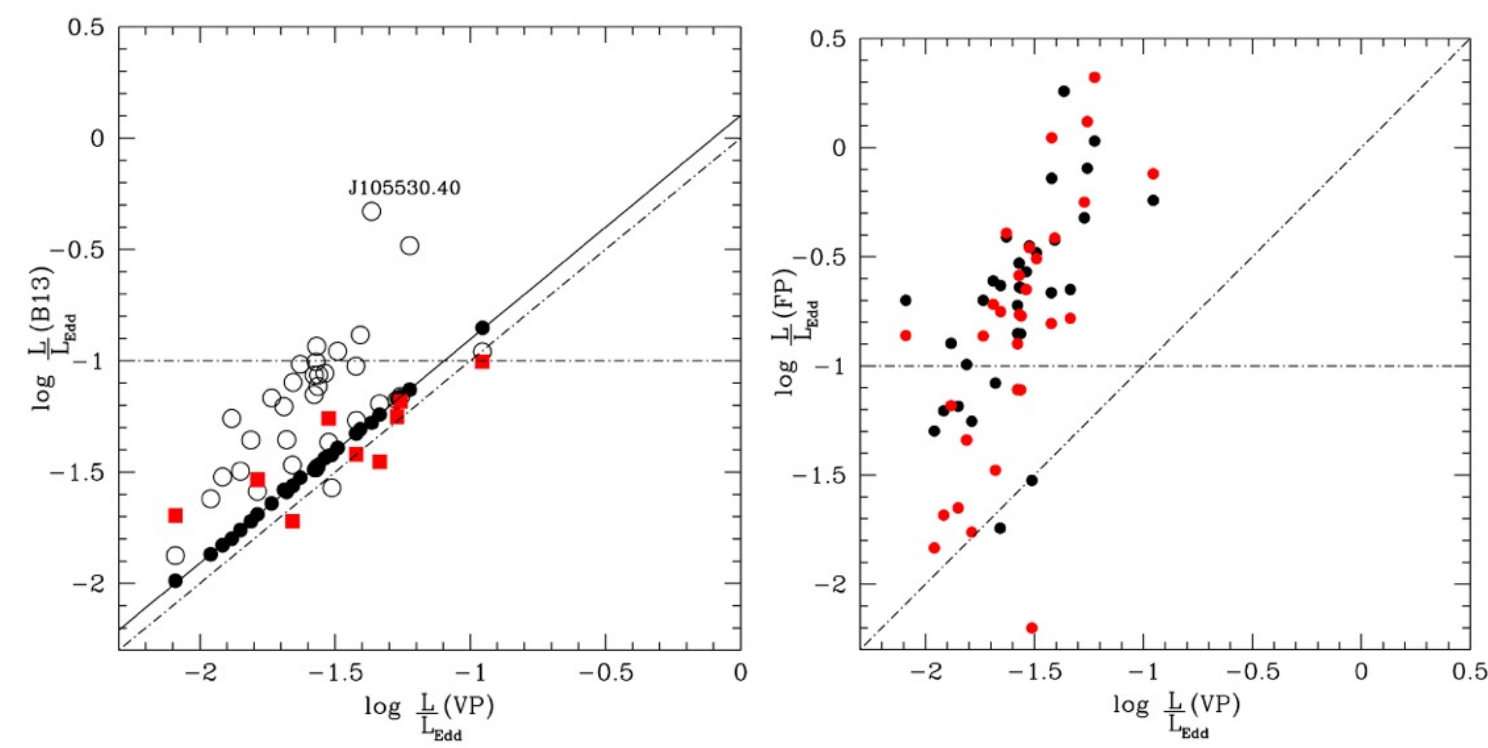

Fig. 10. Left: relation between the Eddington ratio with $M_{\mathrm{BH}}$ computed from the scaling law of VP and with the $r_{\mathrm{BLR}}$ from Bentz et al. (2013) employing an uncorrected FWHM value (black circles). The open circles represent estimates using $f_{2}^{\star}$ (Eq. (4)) and $r_{\mathrm{BLR}}$ corrected following Eq. (5). Red squares represent $L / L_{\text {Edd }}$ estimates from the McConnell et al. (2011) scaling law, with the restriction of $\sigma_{\star} \gtrsim 150 \mathrm{~km} \mathrm{~s}^{-1}$. The oblique dot-dashed line is the equality line; the horizontal one marks the conventional limit separating Pop. A and Pop. B. The filled line is the result of an unweighted least squares fitting between the VP and the uncorrected Bentz et al. (2013) $M_{\mathrm{BH}}$ estimates. Right: relation between the Eddington ratio with $M_{\mathrm{BH}}$ computed from the scaling law of VP and the fundamental plane of Du et al. (2016a) (black circles), and after the refit of this paper (red circles).

cases no orientation effects are considered. Typical uncertainties in the $M_{\mathrm{BH}}$ are expected to be $\approx 0.3$ dex at $1 \sigma$ (Vestergaard \& Peterson 2006; Marziani et al. 2019); this is most likely because of differences in $f$ associated with a different structure $(f \lesssim 1$ and $\approx 2$ were derived for Pop. B and Pop. A, respectively; Collin et al. 2006), and with the effect of orientation. The $r_{\mathrm{BLR}}-L$ is also known to be dependent on $\dot{m}$ (Du \& Wang 2019, and references therein). The main source of uncertainty in luminosity estimates at $5100 \AA$ is the continuum placement and the error associated with the decomposition of host continua the AGN. Even if formal errors are low, it is unlikely that the uncertainty is less than $\approx 10 \%$, which we assume as an indicative value. The computation of the bolometric luminosity suffers from the additional scatter associated with the diversity in the AGN SEDs; scatter at $1 \sigma$ could be assumed $\sim 20 \%$ (Elvis et al. 1994; Richards et al. 2006). We expect a dependence of the bolometric correction along the main sequence; more recent estimates suggest a dependence on luminosity, spin, and the dimensionless accretion rate (e.g., Runnoe et al. 2013; Netzer 2019); however, they are relatively untested and were sparsely considered in past work. We assume a bolometric correction of 10 .

\section{3. $L / L_{E d d}$ estimate using the fundamental plane}

A second method to estimate $L / L_{\text {Edd }}$ can be based on the fundamental plane (FP) of accreting black holes described by Du et al. (2016a). Du et al. (2016a) introduce the notion of the fundamental plane of SEAMBHs defined by a bivariate correlation between the parameter $\dot{\mathcal{M}}=\frac{M_{\mathrm{BH}} c^{2}}{L_{\mathrm{Edd}}}$ that is, the dimensionless accretion rate $\dot{m}=\frac{\eta \dot{M} c^{2}}{L_{\mathrm{Edd}}}$ for $\eta=1$ (Du et al. 2015), the Eddington ratio, and the observational parameters $R_{\mathrm{FeII}}$ and D parameter (ratio $F W H M / \sigma$ of $\mathrm{H} \beta$, where $\sigma$ is the velocity dispersion of the broad component of $\mathrm{H} \beta$ ). The FP can then be written as two linear relations between $\log \dot{\mathcal{M}}$ and $L_{\mathrm{L}} / L_{\text {Edd }}$ vs. $\approx a+b \frac{F W H M}{\sigma}+c R_{\mathrm{FeII}}$, where $a, b$, and $c$ are reported by Du et al. (2016a). The identification criteria included in the fundamental plane are consistent with the ones derived from the E1 approach ( $L / L_{\text {Edd }}$ and $\dot{\mathcal{M}}$ increase as the profiles become Lorentzian-like, and $R_{\text {FeII }}$ becomes higher).

To investigate the origin of this disagreement, we considered that the fit provided by Du et al. (2016a) is very good for high Eddington radiators, but it is biased if low $L / L_{\text {Edd }}$ data are considered. The upper panel of Fig. 11 shows that there is a significant residual between data and fit values, which is dependent on $L / L_{\text {Edd }}$ : At a low Eddington ratio, $\log L / L_{\text {Edd }} \sim-2$, the FP plane fit reported by Du et al. (2016a) predicts a value of $L / L_{\text {Edd }}$ almost one order of magnitude systematically higher with respect to the one inferred by the distribution of the data points. The residuals can be fit by a linear function $\left(\delta=\log L / L_{\text {Edd }}-\log L / L_{\text {Edd }}(\mathrm{FP})\right.$ that zeroes the trend in Fig. 11 (red dots), with a post-correction best fitting line consistent with $\delta\left(L / L_{\text {Edd }}\right) \equiv 0$. Applying the correction to the residuals to obtain new values of $L / L_{\text {Edd }}$ we derive this slightly modified equation for the fundamental plane $\log L / L_{\text {Edd }}=\alpha+\beta D+\gamma R_{\text {FeII }} \approx 0.774-1.33 D+1.30 R_{\text {FeII }}$. The estimates with this new law, although lower at the low $L / L_{\text {Edd }}$ do not solve the disagreement between the VP conventional estimates and the FP estimates (Fig 10). The right panel of Fig. 10 shows that the FP $L / L_{\text {Edd }}$ estimates are in large disagreement with respect to the VP and B13 estimates with both the old and new equation for FP:VP estimates are below the once based on FP by more than one order of magnitude. The disagreement is so serious that the highest radiating source with $L / L_{\mathrm{Edd}} \sim 2$, according to the FP has $L / L_{\text {Edd }} \approx 0.04$ following VP, and that it leads to inconsistencies between the MS interpretation and spectral type assignment: the same source would qualify as a Pop. B source (VP) and as an xA (FP). Using the modified VP with the parameters reported above, the only effect is to bring in agreement only 6-7 points at the low- $L / L_{\text {Edd }}$ end. The bulk of the data point remains above the VP estimates by $\approx 1$ dex.

To further investigate the issue, we computed $M_{\mathrm{BH}}$ from the stellar velocity dispersion $\sigma_{\star}$ of the host bulge, using the scaling 

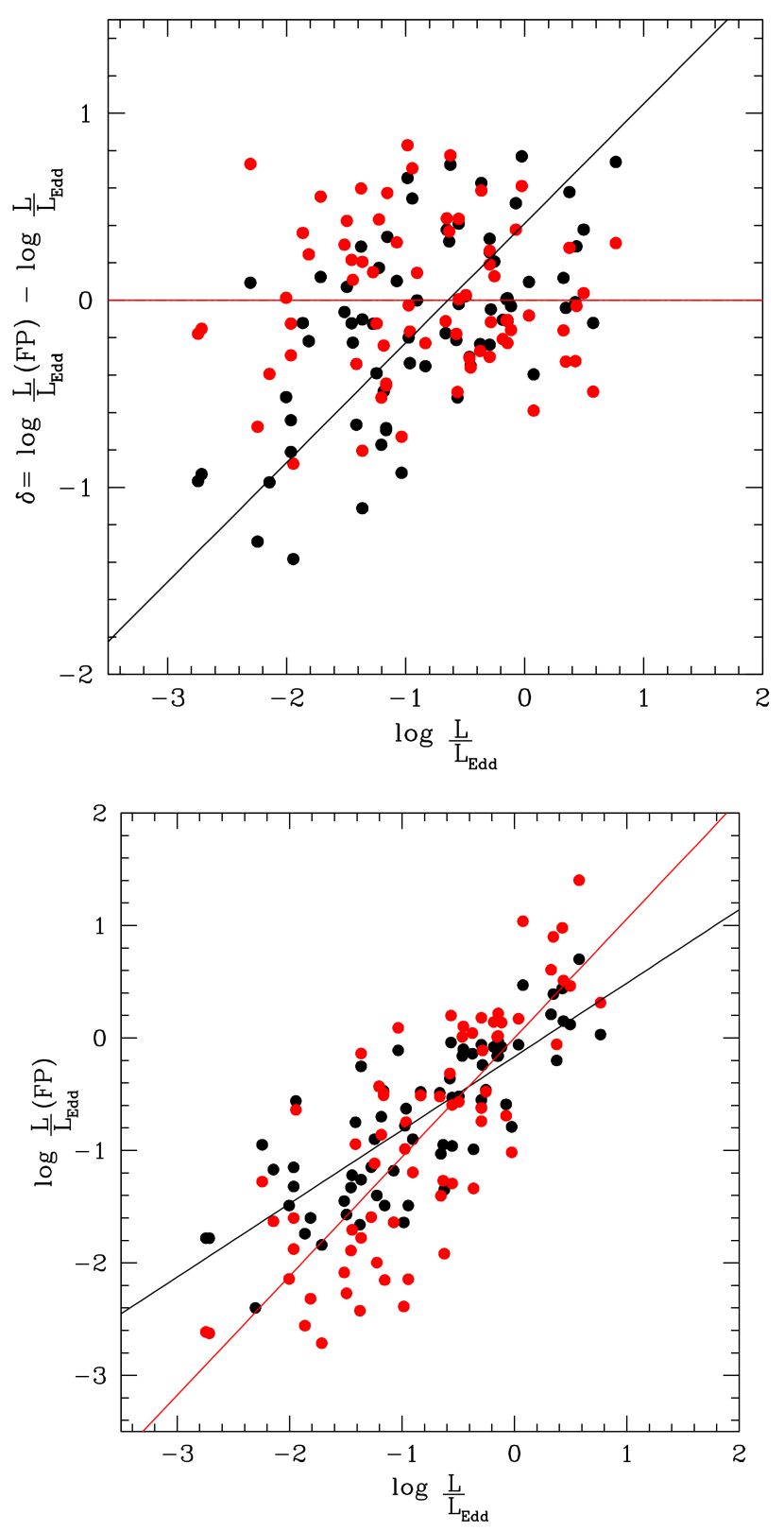

Fig. 11. Improvement on the fundamental plane of accreting black holes. Top panel:5 residuals between the original FP fitting equation of Du et al. (2016a) and the data (black circles). There is a significant linear trend; the black line traces the unweighted least squares best fit. The red circles show the residuals with the modified FP (see Sect. 5.3); there is no trend, and the best fitting (red) line is consistent with the zero slope (black line). Bottom panel: data points with $L / L_{\mathrm{Edd}}$ estimated with the original (black) and revised (red) FP equation.

law $M_{\mathrm{BH}} \approx 1.95 \times 10^{8}\left(\sigma_{\star} / 200\right)^{5.12} M_{\odot}($ McConnell et al. 2011$)$, which is an updated formulation of the original scaling law of Ferrarese \& Merritt (2000). Figure 12 shows that the VP $M_{\mathrm{BH}}$ and $M_{\mathrm{BH}}$ from the host show systematic differences that are strongly correlated with $\sigma_{\star}$, which increase with decreasing $\sigma_{\star}$. The unweighted least squares fitting line shown in Fig. 12 represents a highly significant but likely spurious correlation. When physical velocity dispersion is of the same order or smaller than the instrumental velocity dispersion, it is advisable (Koleva et al. 2009a) to inject the line spread function (LSF) of the spectrograph in the model of the SSP in order to adjust the resolution of the spectra and the model. We refit the spectra where $\sigma_{\star}$ was
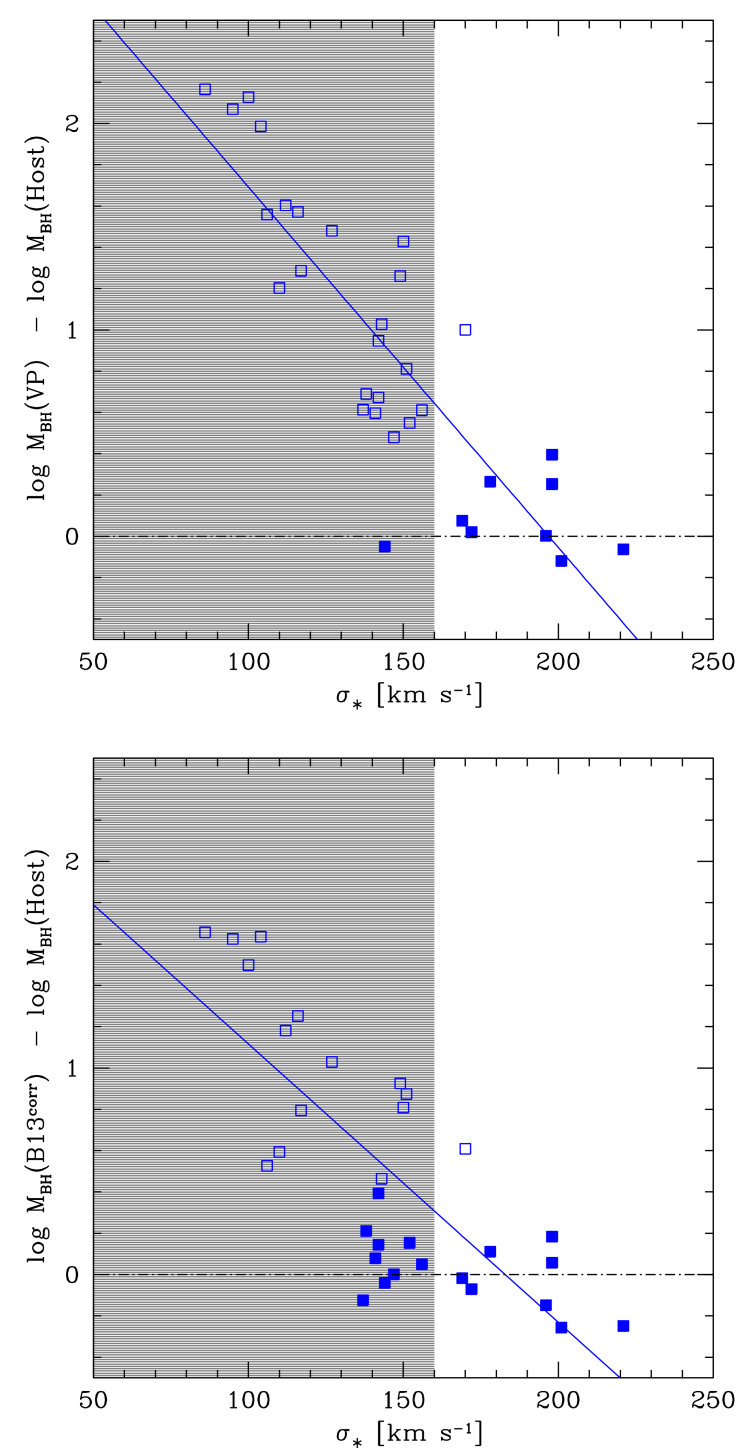

Fig. 12. Top: relation between velocity dispersion $\sigma_{\star}$ and difference between $M_{\mathrm{BH}}$ estimates from $\sigma_{\star}$ using the scaling law of McConnell et al. (2011) and the scaling law from VP. The shaded area identifies the range of $\sigma_{\star} \leq 160 \mathrm{~km} \mathrm{~s}^{-1}$. All data points, save one yielding large systematic differences ( $>0.4$, represented with open squares) with respect to the VP $M_{\mathrm{BH}}$, are within the shaded area. Bottom: same as in the top panel, but with the $M_{\mathrm{BH}}$ values computed with the correction described in Sect. 5.4.

below $150 \mathrm{~km} \mathrm{~s}^{-1}$, with injected LSF in the SSP model, but the restored $\sigma_{\star}$ was just slightly higher then the first estimation of $\sigma_{\star}$, and it is still within the error bars of the first estimation. Therefore, we concluded that LSF injection would not solve the problem of discrepancies between two estimations of the masses. There is a possibility, discussed in Sect. 5.4, that $\sigma_{\star}$ is associated with systems observed face-on, which are therefore also affected by orientation effects.

The FP estimates are based on two parameters that do not include information on line broadening. The parameter $D$ is somewhat redundant as the shape of the $\mathrm{H} \beta$ profile is known to be a MS correlate: the profiles are Gaussian-like $(D \approx 2.3)$ in Pop. B, while they become Lorentzian-like profile in Pop. A (spectral type 1) and are consistent with Lorentzian-like up to the highest $R_{\text {FeII }}$ values, albeit with a blueshifted excess interpreted as Balmer emission from a high-ionization wind, which is more 
easily detected in high-ionization lines such as CIV $\lambda 1549 \AA$ (e.g., Richards et al. 2011). Therefore the behavior of parameter $D$ is not expected to be monotonic along the sequence: It should increase from extreme Pop. B toward A1, where the most Lorentzian-like profiles are observed, and decrease again where a blueshift excess provides a significant deviation from a Lorentzian profile (ST A3 and A4). In addition, one could wonder about the prediction of passing from A2 to B2 and from A3 to $\mathrm{B} 3$ according to the fundamental plane. The $\mathrm{xA}$ sources of $\mathrm{A} 3$ in the sample of Du et al. (2016b) show a typical $D \approx 1.5$; in B3 the profiles are more Gaussian-like, and we can assume a conservative $D \approx 2$; for the same average $R_{\mathrm{FeII}}=1.25$, the change in $L / L_{\text {Edd }}$ would be more than a factor of 2.5 . These considerations focus the issue on the nature of Pop. B2 and B3. Populations B2 and $\mathrm{B} 3$ are rare at low $z$ (B2 are $\lesssim 3 \%$ in the sample of Marziani et al. 2013a; B3 is not even detected, which implies a prevalence of $\lesssim 0.2 \%$ ), and they represent poorly understood classes. There is a degeneracy between effects of orientation and $M_{\mathrm{BH}}$ in the optical plane of the MS; for a fixed $M_{\mathrm{BH}}$, A2 sources seen at higher inclination may be displaced in B2 (Panda et al. 2019). At the same time, we cannot exclude that higher $M_{\mathrm{BH}}$ sources are located within B2. In both cases, for a fixed luminosity, we expect a significant decrease in $L / L_{\text {Edd }}$ passing from B2 to A2.

Furthermore, the object could appear as B2 type, due to a different response of the $\mathrm{H} \beta$ and Fe II flux to the variability of ionizing continuum. Higher $R_{\mathrm{FeII}}$ could be caused by two variability effects as follows: (1) a faster response of $\mathrm{H} \beta$ flux to the variability of ionizing continuum; and (2) a larger amplitude of $\mathrm{H} \beta$ flux variations compared to the amplitude of Fe II flux variations (see e.g., Hu et al. 2015; Barth et al. 2013). Observing single epoch spectra, depending on the variability state of both effects, together with the line width response to the flux variations could contribute to estimates of mass and $L / L_{\text {Edd. }}$. Also, these effects could produce the trend of $L / L_{\text {Edd }}$ decreasing with $R_{\mathrm{FeII}}$ (Bon et al. 2018), which is opposite to the trend along EV1 in which $L / L_{\text {Edd }}$ increases with $R_{\text {FeII }}$ (Marziani et al. 2013b).

\subsection{Orientation and physical parameter estimates}

The previous analysis ignored the effect of the orientation on the $M_{\mathrm{BH}}$ computation. However, growing evidence suggests that the low-ionization lines-emitting BLR is highly flattened (e.g., Mejía-Restrepo et al. 2018, and references therein). If this is the case, the observed velocity can be parameterized as $\delta v_{\mathrm{obs}}^{2}=$ $\delta v_{\text {iso }}^{2} / 3+v_{\mathrm{Kepl}}^{2} \sin ^{2} \theta$, and if $\delta v_{\text {iso }} / \delta v_{\mathrm{Kepl}} \approx 0.1$, where $\delta v_{\text {iso }}$ is an isotropic velocity component, and $\delta v_{\mathrm{Kepl}}$ the Keplerian velocity. For a geometrically thin disk, it implies $\delta v_{\mathrm{obs}} \approx \delta v_{\mathrm{Kepl}} / \sin \theta$ if the FWHM is taken as the $\delta v_{\mathrm{obs}}$, and $\delta v_{\mathrm{Kepl}}=0$, that is, in the case of isotropic velocity dispersion, $f_{2}=\frac{3}{4}$. If the VBE estimates are not corrected beforehand for orientation, the structure factor is $f_{2} \propto 1 / \sin ^{2} \theta$ (e.g., McLure \& Dunlop 2001; Jarvis $\&$ McLure 2006; Decarli et al. 2011), and more precisely, we assume $f_{1} \equiv 1$ :

$$
f_{2}=\frac{1}{4\left[\frac{1}{3}\left(\frac{\delta v_{\text {iso }}}{\delta v_{\mathrm{K}}}\right)^{2}+\sin ^{2} \theta\right]} .
$$

We attempt to consider the effect of the viewing angle on the $\mathrm{H} \beta$ line width by considering that the virial factor is anticorrelated with the FWHM of the broad emission line. For the $\mathrm{H} \beta$ line the relation is given by

$f_{2}^{\star}=\left(\frac{F W H M}{4550}\right)^{-1.17}$,
(Mejía-Restrepo et al. 2018). This implies that sources with an $F W H M \mathrm{H} \beta$ narrower than $4550 \mathrm{~km} \mathrm{~s}^{-1}$ should have their mass increased by a factor that can be as large as $\approx 5$ in the case of the narrowest $\mathrm{H} \beta$ profiles observed in NLSy1s. The effect is milder than the one predicted in Eq. (3), and it may be better suited for the general population of quasars encompassing both typical Pop. A and B sources. In addition, Martínez-Aldama et al. (2019) suggest a correction to the B13 $r_{\mathrm{BLR}}$ estimate, following the reverberation mapping campaign of highly accreting quasars (Du et al. 2018, and references therein), $\delta r_{\mathrm{BLR}}=\log \left(r_{\mathrm{BLR}} / r_{\mathrm{BLR}, \mathrm{B} 13}\right)$. According to Martínez-Aldama et al. (2019), with the $f_{2}^{\star}$ dependence on the FWHM, the correction to $r_{\mathrm{BLR}}$ is:

$\delta r_{\mathrm{BLR}}=(-0.271 \pm 0.030) \log \frac{L_{\mathrm{bol}}}{L_{\mathrm{Edd}}^{\star}}+(-0.396 \pm 0.032)$,

where $L_{\text {Edd }}^{\star}$ means that the Eddington luminosity has been computed with virial mass relation assuming $f_{2}^{\star}$ (Eq. (4)). The $L / L_{\text {Edd }}$ values computed with this approach deviate significantly from the VP and B13 $L / L_{\text {Edd }}$, yielding a higher value of $L / L_{\text {Edd }}$ (Fig. 10), because most of the sources have an $F W H M(\mathrm{H} \beta) \gtrsim 4500$, and hence $f^{\star}<1$, which implies a lower $M_{\mathrm{BH}}$. The effect visible in Fig. 10 comes mostly from the $f_{2}^{\star}$ : The $\delta r_{\mathrm{BLR}}$ is small since $L / L_{\mathrm{Edd}}$ is low $\left(\log L / L_{\mathrm{Edd}} \sim\right.$ $-1 .-1.5)$. The agreement between the modified VP and the FP remains poor. After the corrections, however, the xA candidate SDSS J105530.40+132117.7 is recognized as the highest radiator, with $L / L_{\mathrm{Edd}} \approx-0.35$, which is close to the conventional lower limit for $\mathrm{xA}$ sources. We can conclude that the objects in our sample are safely not xA (apart from the one source mentioned directly above, and perhaps a couple of borderline cases). The question that arises is whether they are Pop. A. Following the FP, all of them save seven should be of Pop. A, with five $x A$ candidates (Fig. 10). Following the modified expression of Eq. (4), about one half of the sources have $\log L / L_{\text {Edd }} \gtrsim-1$, the conventional boundary between Pop. A and Pop. B.

If we consider the modified viral mass as described in the previous paragraph, any correlation with the McConnell et al. (2011) remains fairly weak $(r \approx 0.385$, significant at just $2 \sigma$ confidence level). However, the bottom panel of Fig. 12 shows that now more than half of the sample has masses in reasonable agreement with ones from the host. For the remaining sources with small $\sigma_{\star}$, we consider that bulges seen face-on should be affected by orientation in their measured velocity dispersion, as they are rotationally supported (Kormendy \& Illingworth 1982). Testing this possibility goes beyond the goals of the present paper.

The existence of a relation between $R_{\mathrm{FeII}}$ and $L / L_{\mathrm{Edd}}$ is a robust result and rests on several lines of evidence (Grupe et al. 1999; Kuraszkiewicz et al. 2004; Dong et al. 2011; Marziani et al. 2013b; Sun \& Shen 2015, in addition to the analysis of Du et al. 2016b; Du \& Wang 2019). For instance Marziani et al. (2013b) show (their Fig. 6) that there is a $L / L_{\text {Edd }}$ systemic trend along Pop. A, with $L / L_{\text {Edd }}$ increasing with $R_{\text {FeII }}$. Independent evidence is provided by Sun \& Shen (2015): For the luminosity in a fixed range, the $\sigma_{\star}$ (a proxy of $M_{\mathrm{BH}}$ ) decreases systematically with increasing $R_{\mathrm{FeII}}$ up to $R_{\mathrm{FeII}} \sim 1$. Indirect evidence is also provided by one of the correlations of 4DE1: The highest correlation coefficient is between $R_{\text {FeII }}$ and CIV $\lambda 1549 \AA$ blueshift amplitude (Sulentic et al. 2000c, 2007, 2017). The balance between radiative and gravitational forces is able to account for a large part of the quasars optical and UV phenomenology along the quasar MS (Ferland et al. 2009). Therefore the key 
factor in the discrepancy of the $L / L_{\mathrm{Edd}}$ estimates around A2/B2 is most likely the orientation, as the viewing angle $\theta$ affects $F W H M(\mathrm{H} \beta)$ linearly, and $M_{\mathrm{BH}}$ quadratically. By the same token a large fraction of the $\mathrm{B} 2$ sources could be very well intrinsically Pop. A, especially the ones with $R_{\text {FeII }}$ significantly above 0.5 and the FWHM borderline.

Great care should be used in the computation of the $M_{\mathrm{BH}}$ and $L / L_{\mathrm{Edd}}$ if no orientation correction is possible. As a test not related to the present sample, we considered the sources of the Du et al. (2016b) sample that are $\mathrm{xA}$, that is, satisfying the criterion $R_{\text {FeII }}>1.2$ following Negrete et al. (2018). For these six sources the $\log L / L_{\text {Edd }}$ average value applying the original FP is $\approx 0.45$, implying that they should be considered true "super-Eddington" accreting massive black holes (SEAMBHs, Wang et al. 2014a). For these xA sources, it is possible to derive an estimate of the viewing angle $\theta$ (Negrete et al. 2018): The difference between the virial luminosity estimate and the concordance luminosity (assumed to be the correct luminosity) is expected to be mainly dependent on the viewing angle, which is assumed to strongly affect the FWHM according to Eq. (3). For these sources, $\theta \sim 0.2 \mathrm{rad}$ : their emitting regions are expected to be seen almost face-on. If the $M_{\mathrm{BH}}$ are recalculated following Eqs. (2) and (3) with the $\theta$ estimated following Negrete et al. (2018), the $\log L / L_{\text {Edd }}$ is lowered to an average of $\approx-0.147$, which is consistent with the estimates using the conventional approach (Marziani \& Sulentic 2014). The Negrete et al. (2018) approach cannot be extended to the sample of the present paper, but it is a strong indication that $L / L_{\text {Edd }}$ actual values depend on a normalization factor that is in turn dependent on the viewing angle via the dependence on the viewing angle of $M_{\mathrm{BH}}$. The xA sources apparently radiate at a limiting Eddington ratio along the MS. However, it is unclear whether the xA sources are truly SEAMBHs. Even if there is consistency in the selection criteria, their Eddington ratios and $\dot{\mathcal{M}}$ are much too affected by uncertainties in the $M_{\mathrm{BH}}$ to be a safe discriminant.

\section{Conclusions}

This paper has analyzed a spin-off of the Negrete et al. (2018) sample and precisely evaluated spectra that were preliminarily selected as xA candidates and that afterwards were found to suffer strong contamination by the spectrum of the host galaxy. The main results encompass the following:

1. A proper identification of $\mathrm{xA}$ sources requires a careful simultaneous multicomponent fit in order to retrieve information on the stellar continuum and on the FeII emission, especially if the AGN is of low luminosity, and the data are from optical fiber with a relatively large angular coverage. Inclusion of the spurious xA sources should be avoided since in cosmological studies it may dramatically increase the dispersion in the Hubble diagram of quasars obtained from virial luminosity estimates.

2. Objects of our sample with strong host galaxy contamination show properties that suggest more modest activity, in comparison with xA sources. Modest activity means that they lack extreme outflows, strong starburst activity, and a high accretion rate, which are typical of $\mathrm{xA}$ sources.

3. We find a high fraction of host galaxy spectrum (in half of the sample even higher than $40 \%$ ). We conclude that when absorption lines are prominent, and the fraction of the host galaxy is high, SSP mimics FeII, and that this can lead to mistaken identification of FeII spectral features. We have identified several stellar absorption lines that, along with the continuum shape, may lead to an overestimate of $R_{\text {FeII }}$, and therefore to the misclassification of sources as xA sources. Our results lend support to the results of Śniegowska et al. (2018) who find that only six sources out of a sample of 23 could be classified as $\mathrm{xA}$ after a careful decomposition of all spectral components that also involve the spectrum of the host galaxy.

4. We have studied the 32 sources with high host galaxy contamination as an independent sample which has an interest of its own. We have used a host galaxy shift as a reference frame in order to study shifts of emission lines more precisely. Unlike xA sources, there is very good agreement between the shift of absorption spectrum and the shifts of $\mathrm{H} \beta_{\mathrm{NC}}$, [OII] $\lambda 3727$, and the [OIII] $\lambda 5007$. The good agreement between [OIII] $\lambda 5007$ and the narrow low-ionization lines $\mathrm{H} \beta_{\mathrm{NC}}$ and [OII] $\lambda 3727$ has important consequences for the systemic redshift estimates in case no host absorptions could be detected.

5. We have considered the effect of the density on the effective wavelength of the $[\mathrm{OII}] \lambda 3727$ doublet, providing a relation linking $n_{\mathrm{H}}$ and $\lambda_{\mathrm{eff}}$. The dependence on density introduces a significant error in [OII] $\lambda 3727$-based redshift estimates. However, the effective wavelength density dependence could, in principle, provide a diagnostics of the relative importance of the AGN NLR and of circum-nuclear star formation producing low-density HII regions.

6. We find mostly old SSP (older than 1 Gyr) for the HG. The metallicities of SSPs in our sample of 32 sources are mainly solar.

7. The HG sources cluster around spectral type B2. Considering the spectral type correlation with $L / L_{\mathrm{Edd}}$, a large fraction of them should be considered to be inclined Pop. A sources.

8. Computations of $M_{\mathrm{BH}}$ are problematic, especially if small samples of heterogeneous sources involving a broad range of $\mathrm{H} \beta F W H M$ are considered (see e.g., Shen 2013). We have discussed estimates of $M_{\mathrm{BH}}$ and $L / L_{\mathrm{Edd}}$, and we emphasized the effect of orientation that should be considered if a meaningful comparison of $L / L_{\text {Edd }}$ values between sources of widely different width has to be done. In principle, if viewing angles were known for each source, the $M_{\mathrm{BH}}$ and $L / L_{\mathrm{Edd}}$ values could be normalized to a standard $\theta$, which would then let physical trends emerge more clearly. Individual $\theta$ estimates are still unavailable for the general population of quasars outside of the MS extremes such as the ones considered in this study, although $\theta$ computations may become widespread in the coming years. Spectropolarimetric measurements, even if they are demanding in terms of telescope time, have provided individual $\theta$ values for sources in different spectral types along the MS (Afanasiev \& Popović 2015). Other techniques, based on the SED also show promising possibilities (Capellupo et al. 2015; Mejía-Restrepo et al. 2018).

9. At this point, one has to consider that the VP scaling law, which is perfectly consistent with the use of the more recent Bentz et al. (2013) $r_{\mathrm{BLR}}$ scaling law, is biased and in favor of broader sources (i.e., the many Population B sources that were targets of early reverberation mapping campaigns). On the converse, the $L / L_{\text {Edd }}$ estimates from the Du et al. (2016a) fundamental planes are apparently biased toward narrow sources (i.e., the many NLSy1s and Population A sources that are included in the Du et al. 2016a sample). In both cases, a small FWHM is taken as a synonym of small $M_{\mathrm{BH}}$, and a broad FWHM is considered to be a synonym of large $M_{\mathrm{BH}}$. Presently, we know this is not the case because of the degeneration between mass and orientation effects. The present work draws attention to how VP 
overestimate the $M_{\mathrm{BH}}$, underestimate $L / L_{\mathrm{Edd}}$, and how Du et al. (2016a) underestimate the $M_{\mathrm{BH}}$, and overestimate $L / L_{\text {Edd }}$. These problems are likely to be overcome by the next-generation SDSS-V panoptic spectroscopy (Kollmeier et al. 2017), multi-epoch spectroscopic survey of over six million objects that plans reverberation-mapping quality monitoring for thousands of quasars.

The huge range in luminosity covered by accretion phenomena in the nuclei of galaxies and the apparent lack of any strong luminosity correlation with observational properties have hampered the exploitation of quasars as "standard candles". The analysis presented in this paper will be instrumental to the definition of a sample of xA sources, which are believed to show stable, selfsimilar properties that could make them suitable for distance measurements in cosmology.

Acknowledgements. This research is part of the projects 176001 "Astrophysical spectroscopy of extragalactic objects" and 176003 "Gravitation and the large scale structure of the Universe", funded by Ministry of Education, Science and Technological Development of the Republic of Serbia. PM and MDO acknowledge funding from the INAF PRIN-SKA 2017 program 1.05.01.88.04 PM also acknowledges the Programa de Estancias de Investigación (PREI) No. DGAP/DFA/2192/2018 of UNAM. A.d.O. acknowledges financial support from the Spanish Ministry of Economy and Competitiveness through grant AYA2016-76682-C3-1-P and from the State Agency for Research of the Spanish MCIU through the "Center of Excellence Severo Ochoa" award for the Instituto de Astrofísica de Andalucía (SEV-2017-0709). ML. M. A acknowledges financial support of National Science Centre, Poland, grant No. 2017/26/A/ST9/00756 (Maestro 9). DD acknowledges support from grants PAPIIT, UNAM 113719, Mexico D.F. 04510, Mexico. AN acknowledges support from grant CONACyT research fellow - Instituto de Astronomía, UNAM, Mexico D.F. 04510, Mexico. We thank to Pu Du for his help and constructive comments. Funding for the SDSS and SDSS-II has been provided by the Alfred P. Sloan Foundation, the Participating Institutions, the National Science Foundation, the U.S. Department of Energy, the National Aeronautics and Space Administration, the Japanese Monbukagakusho, the Max Planck Society, and the Higher Education Funding Council for England. The SDSS Web Site is http: //www . sdss.org/. The SDSS is managed by the Astrophysical Research Consortium for the Participating Institutions. The Participating Institutions are the American Museum of Natural History, Astrophysical Institute Potsdam, University of Basel, University of Cambridge, Case Western Reserve University, University of Chicago, Drexel University, Fermilab, the Institute for Advanced Study, the Japan Participation Group, Johns Hopkins University, the Joint Institute for Nuclear Astrophysics, the Kavli Institute for Particle Astrophysics and Cosmology, the Korean Scientist Group, the Chinese Academy of Sciences (LAMOST), Los Alamos National Laboratory, the Max-Planck-Institute for Astronomy (MPIA), the Max-Planck-Institute for Astrophysics (MPA), New Mexico State University, Ohio State University, University of Pittsburgh, University of Portsmouth, Princeton University, the United States Naval Observatory, and the University of Washington.

\section{References}

Abramowicz, M. A., \& Straub, O. 2014, Scholarpedia, 9, 2408

Abramowicz, M. A., Czerny, B., Lasota, J. P., \& Szuszkiewicz, E. 1988, ApJ, 332,646

Afanasiev, V. L., \& Popović, L. Č. 2015, ApJ, 800, L35

Barth, A. J., Pancoast, A., Bennert, V. N., et al. 2013, ApJ, 769, 128

Bennert, V. N., Loveland, D., Donohue, E., et al. 2018, MNRAS, 481, 138

Bensch, K., del Olmo, A., Sulentic, J., Perea, J., \& Marziani, P. 2015, J. Astrophys. Astron., 36, 467

Bentz, M. C., Denney, K. D., Grier, C. J., et al. 2013, ApJ, 767, 149

Berton, M., Caccianiga, A., Foschini, L., et al. 2016, A\&A, 591, A98

Bian, W.-H., Fang, L.-L., Huang, K.-L., \& Wang, J.-M. 2012, MNRAS, 427, 2881

Boller, T., Brandt, W. N., \& Fink, H. 1996, A\&A, 305, 53

Bon, N., Popović, L. Č., \& Bon, E. 2014, AdSpR, 54, 1389

Bon, N., Bon, E., Marziani, P., \& Jovanović, P. 2015, Ap\&SS, 360, 7

Bon, E., Zucker, S., Netzer, H., et al. 2016, ApJS, 225, 29

Bon, N., Bon, E., \& Marziani, P. 2018, Front. Astron. Space Sci., 5, 3

Boroson, T. A. 2002, ApJ, 565, 78

Boroson, T. A., \& Green, R. F. 1992, ApJS, 80, 109
Brightman, M., Bachetti, M., Earnshaw, H. P., et al. 2019, Bull. Am. Astron. Soc., 51, 352

Canto, J., Meaburn, J., Theokas, A. C., \& Elliott, K. H. 1980, MNRAS, 193, 911 Capellupo, D. M., Netzer, H., Lira, P., Trakhtenbrot, B., \& Mejía-Restrepo, J. 2015, MNRAS, 446, 3427

Cid Fernandes, R., Mateus, A., Sodré, L., Stasińska, G., \& Gomes, J. M. 2005, MNRAS, 358, 363

Collin, S., Kawaguchi, T., Peterson, B. M., \& Vestergaard, M. 2006, A\&A, 456, 75

Condon, J. J., Hutchings, J. B., \& Gower, A. C. 1985, AJ, 90, 1642

Cracco, V., Ciroi, S., Berton, M., et al. 2016, MNRAS, 462, 1256

Czerny, B., Beaton, R., Bejger, M., et al. 2018, Space Sci. Rev., 214, 32

Decarli, R., Dotti, M., \& Treves, A. 2011, MNRAS, 413, 39

Dewangan, G. C., Singh, K. P., Gunn, K. F., et al. 2002, MNRAS, 337, 693

Dimitrijević, M. S., Popović, L. Č., Kovačević, J., Dačić, M., \& Ilić, D. 2007, MNRAS, 374, 1181

Dong, X.-B., Wang, J.-G., Ho, L. C., et al. 2011, ApJ, 736, 86

D'Onofrio, M., \& Burigana, C. 2009, Questions of Modern Cosmology: Galileo's Legacy (Berlin: Springer Verlag)

Du, P., \& Wang, J.-M. 2019, ApJ, 886, 42

Du, P., Hu, C., Lu, K.-X., et al. 2015, ApJ, 806, 22

Du, P., Wang, J.-M., Hu, C., et al. 2016a, ApJ, 818, L14

Du, P., Lu, K.-X., Hu, C., et al. 2016b, ApJ, 820, 27

Du, P., Zhang, Z.-X., Wang, K., et al. 2018, ApJ, 856, 6

Elvis, M., Wilkes, B. J., McDowell, J. C., et al. 1994, ApJS, 95, 1

Eracleous, M., \& Halpern, J. P. 2004, ApJS, 150, 181

Euclid Red Book Editorial Team 2011, Euclid: definition study report No. $\operatorname{SRE}(2011) 12$ (ESA)

Ferland, G. J., Korista, K. T., Verner, D. A., et al. 1998, PASP, 110, 761

Ferland, G. J., Hu, C., Wang, J., et al. 2009, ApJ, 707, L82

Ferrarese, L., \& Merritt, D. 2000, ApJ, 539, L9

Ganci, V., Marziani, P., D’Onofrio, M., et al. 2019, A\&A, 630, A110

Gaur, H., Gu, M., Ramya, S., \& Guo, H. 2019, A\&A, 631, A46

Ge, X., Bian, W.-H., Jiang, X.-L., Liu, W.-S., \& Wang, X.-F. 2016, MNRAS, 462, 966

Gebhardt, K., Bender, R., Bower, G., et al. 2000, ApJ, 539, L13

Grupe, D., Beuermann, K., Mannheim, K., \& Thomas, H.-C. 1999, A\&A, 350, 805

Grupe, D., Komossa, S., Leighly, K. M., \& Page, K. L. 2010, ApJS, 187, 64

Heckman, T. M., Miley, G. K., van Breugel, W. J. M., \& Butcher, H. R. 1981, ApJ, 247, 403

Hook, I. M. 2013, Phil. Trans. R. Soc. London Ser. A, 371, 20282

Hu, C., Du, P., Lu, K.-X., et al. 2015, ApJ, 804, 138

Jarvis, M. J., \& McLure, R. J. 2006, MNRAS, 369, 182

Jiang, L., Fan, X., Ivezić, Ž., et al. 2007, ApJ, 656, 680

Kaasinen, M., Bian, F., Groves, B., Kewley, L. J., \& Gupta, A. 2017, MNRAS, 465, 3220

Kakkad, D., Groves, B., Dopita, M., et al. 2018, A\&A, 618, A6

Kauffmann, G., \& Maraston, C. 2019, MNRAS, 489, 1973

Kellermann, K. I., Sramek, R., Schmidt, M., Shaffer, D. B., \& Green, R. 1989, AJ, 98, 1195

Kniazev, A. Y., Pustilnik, S. A., Grebel, E. K., Lee, H., \& Pramskij, A. G. 2004, ApJS, 153, 429

Koleva, M., Prugniel, P., Ocvirk, P., Le Borgne, D., \& Soubiran, C. 2008, MNRAS, 385, 1998

Koleva, M., Prugniel, P., Bouchard, A., \& Wu, Y. 2009a, A\&A, 501, 1269

Koleva, M., Prugniel, P., De Rijcke, S., Zeilinger, W. W., \& Michielsen, D. 2009b, Astron. Nachr., 330, 960

Kollmeier, J. A., Zasowski, G., Rix, H. W., et al. 2017, ArXiv e-prints [arXiv:1711.03234]

Komossa, S., \& Xu, D. 2007, ApJ, 667, L33

Komossa, S., Xu, D., Zhou, H., Storchi-Bergmann, T., \& Binette, L. 2008, ApJ, 680,926

Kormendy, J., \& Ho, L. C. 2013, ARA\&A, 51, 511

Kormendy, J., \& Illingworth, G. 1982, ApJ, 256, 460

Kriss, G. 1994, in Astronomical Data Analysis Software and Systems III, ASP Conf. Ser., 61, 437

Kuraszkiewicz, J. K., Green, P. J., Crenshaw, D. M., et al. 2004, ApJS, 150, 165

Lawson, C. L., \& Hanson, R. J. 1995, Solving Least Squares Problems, Society for Industrial and Applied Mathematics

Letawe, G., Magain, P., Courbin, F., et al. 2007, MNRAS, 378, 83

Maddox, N. 2018, MNRAS, 480, 5203

Marquardt, D. W. 1963, J. Soc. Ind. Appl. Math., 11, 431

Martínez-Aldama, M. L., Del Olmo, A., Marziani, P., et al. 2018, Front. Astron. Space Sci., 4, 65

Martínez-Aldama, M. L., Czerny, B., Kawka, D., et al. 2019, ApJ, 883, 170

Marziani, P., \& Sulentic, J. W. 2012, New Astron. Rev., 56, 49

Marziani, P., \& Sulentic, J. W. 2014, MNRAS, 442, 1211 
Marziani, P., Sulentic, J. W., Zwitter, T., Dultzin-Hacyan, D., \& Calvani, M. 2001, ApJ, 558, 553

Marziani, P., Sulentic, J. W., Stirpe, G. M., Zamfir, S., \& Calvani, M. 2009, A\&A 495, 83

Marziani, P., Sulentic, J. W., Negrete, C. A., et al. 2010, MNRAS, 409, 1033

Marziani, P., Sulentic, J. W., Plauchu-Frayn, I., \& del Olmo, A. 2013a, A\&A, 555, A89

Marziani, P., Sulentic, J. W., Plauchu-Frayn, I., \& del Olmo, A. 2013b, ApJ, 764, 150

Marziani, P., Martínez Carballo, M. A., Sulentic, J. W., et al. 2016, Ap\&SS, 361, 29

Marziani, P., Dultzin, D., Sulentic, J. W., et al. 2018, Front. Astron. Space Sci., 5,6

Marziani, P., del Olmo, A., Martínez-Carballo, M. A., et al. 2019, A\&A, 627, A88

McConnell, N. J., Ma, C.-P., Gebhardt, K., et al. 2011, Nature, 480, 215

McLure, R. J., \& Dunlop, J. S. 2001, MNRAS, 327, 199

Mejía-Restrepo, J. E., Lira, P., Netzer, H., Trakhtenbrot, B., \& Capellupo, D. M. 2018, Nat. Astron., 2, 63

Mineshige, S., Kawaguchi, T., Takeuchi, M., \& Hayashida, K. 2000, PASJ, 52, 499

Mirabel, I. F., \& Sanders, D. B. 1988, ApJ, 335, 104

Moore, C. E. 1945, Contrib. Princeton Univ. Obs., 20, 1

Morton, D. C. 1991, ApJS, 77, 119

Moultaka, J. 2005, A\&A, 430, 95

Negrete, C. A., Dultzin, D., Marziani, P., et al. 2018, A\&A, 620, A118

Nelson, C. H., \& Whittle, M. 1996, ApJ, 465, 96

Netzer, H. 1990, in Active Galactic Nuclei, eds. R. D. Blandford, H. Netzer, L. Woltjer, T. J. L. Courvoisier, \& M. Mayor, 57

Netzer, H. 2013, The Physics and Evolution of Active Galactic Nuclei (Cambridge: Cambridge University Press)

Netzer, H. 2019, MNRAS, 488, 5185

Ni, Q., Brandt, W. N., Luo, B., et al. 2018, MNRAS, 480, 5184

Osterbrock, D. E., \& Ferland, G. J. 2006, Astrophysics of Gaseous Nebulae and Active Galactic Nuclei (Mill Valley: University Science Books)

Padovani, P. 2017, Front. Astron. Space Sci., 4, 35

Panda, S., Marziani, P., \& Czerny, B. 2019, ApJ, 882, 79

Popović, L. Č., \& Kovačević, J. 2011, ApJ, 738, 68

Pradhan, A. K., \& Nahar, S. N. 2015, Atomic Astrophysics and Spectroscopy (Cambridge: Cambridge University Press)

Punsly, B. 2013, ApJ, 762, L25

Richards, G. T., Kruczek, N. E., Gallagher, S. C., et al. 2011, AJ, 141, 167

Richards, G. T., Lacy, M., Storrie-Lombardi, L. J., et al. 2006, ApJS, 166, 470
Risaliti, G., \& Lusso, E. 2015, ApJ, 815, 33

Risaliti, G., \& Lusso, E. 2019, Nat. Astron., 3, 272

Runnoe, J. C., Shang, Z., \& Brotherton, M. S. 2013, MNRAS, 435, 3251

Sadowski, A. 2011, ArXiv e-prints [arXiv:1108.0396]

Sánchez-Blázquez, P., Peletier, R. F., Jiménez-Vicente, J., et al. 2006, MNRAS, 371,703

Sanders, R. L., Shapley, A. E., Kriek, M., et al. 2016, ApJ, 816, 23

Sani, E., Lutz, D., Risaliti, G., et al. 2010, MNRAS, 403, 1246

Sexton, R. O., Canalizo, G., Hiner, K. D., et al. 2019, ApJ, 878, 101

Shemmer, O., Trakhtenbrot, B., Anderson, S. F., et al. 2010, ApJ, 722, L152

Shen, Y. 2013, Bull. Astron. Soc. India, 41, 61

Shen, Y., \& Ho, L. C. 2014, Nature, 513, 210

Shen, Y., Richards, G. T., Strauss, M. A., et al. 2011, ApJS, 194, 45

Śniegowska, M., Czerny, B., You, B., et al. 2018, A\&A, 613, A38

Sulentic, J. W., Marziani, P., \& Dultzin-Hacyan, D. 2000a, ARA\&A, 38, 521

Sulentic, J. W., Marziani, P., Zwitter, T., Dultzin-Hacyan, D., \& Calvani, M. 2000b, ApJ, 545, L15

Sulentic, J. W., Zwitter, T., Marziani, P., \& Dultzin-Hacyan, D. 2000c, ApJ, 536, L5

Sulentic, J. W., Marziani, P., Zamanov, R., et al. 2002, ApJ, 566, L71

Sulentic, J. W., Bachev, R., Marziani, P., Negrete, C. A., \& Dultzin, D. 2007, ApJ, 666, 757

Sulentic, J. W., del Olmo, A., Marziani, P., et al. 2017, A\&A, 608, A122

Sun, J., \& Shen, Y. 2015, ApJ, 804, L15

Thomas, A. D., Kewley, L. J., Dopita, M. A., et al. 2018, ApJ, 861, L2

Trakhtenbrot, B., \& Netzer, H. 2012, MNRAS, 427, 3081

Vazdekis, A. 1999, ApJ, 513, 224

Véron-Cetty, M.-P., \& Véron, P. 2006, A\&A, 455, 773

Vestergaard, M., \& Peterson, B. M. 2006, ApJ, 641, 689

Wang, T., Brinkmann, W., \& Bergeron, J. 1996, A\&A, 309, 81

Wang, J.-M., Du, P., Valls-Gabaud, D., Hu, C., \& Netzer, H. 2013, Phys. Rev. Lett., 110, 081301

Wang, J.-M., Du, P., Hu, C., et al. 2014a, ApJ, 793, 108

Wang, J.-M., Du, P., Li, Y.-R., et al. 2014b, ApJ, 792, L13

Wang, J.-M., Qiu, J., Du, P., \& Ho, L. C. 2014c, ApJ, 797, 65

Wang, J.-M., Du, P., Brotherton, M. S., et al. 2017, Nat. Astron., 1, 775

Watarai, K.-Y., Fukue, J., Takeuchi, M., \& Mineshige, S. 2000, PASJ, 52, 133

Watson, D., Denney, K. D., Vestergaard, M., \& Davis, T. M. 2011, ApJ, 740, L49

White, R. L., Becker, R. H., Helfand, D. J., \& Gregg, M. D. 1997, ApJ, 475, 479 Zamanov, R., Marziani, P., Sulentic, J. W., et al. 2002, ApJ, 576, L9

Zamfir, S., Sulentic, J. W., Marziani, P., \& Dultzin, D. 2010, MNRAS, 403, 1759

Zhang, K., Dong, X.-B., Wang, T.-G., \& Gaskell, C. M. 2011, ApJ, 737, 71

Zhang, Z.-X., Du, P., Smith, P. S., et al. 2019, ApJ, 876, 49 


\section{Appendix A: SSP analysis atlas}

Figure A.1 shows the appearance of the spectrum in the range $4000-5500 \AA$ where the fit was carried out, a zoom around $\mathrm{H} \beta$ and [OIII] $\lambda \lambda 4959,5007$, and the cleaned $\mathrm{H} \beta$ profile for the sources of the HG sample. The follow-up measurements of the individual spectra obtained after the fitting with ULySS are reported in Table 2. Although, in the case of few spectra, the narrow and semibroad component of [OIII] $\lambda 5007$ lines have switched places; we disentangled the two components according to their widths. Figure A.2 represents the $\chi^{2}$ maps for the entire sample in the parameter space of the stellar velocity dispersion and the mean stellar velocity. 
N. Bon et al.: Narrow emission and absorption lines among Fe $\mathrm{II}_{\mathrm{opt}}$ strong low- $z$ quasars
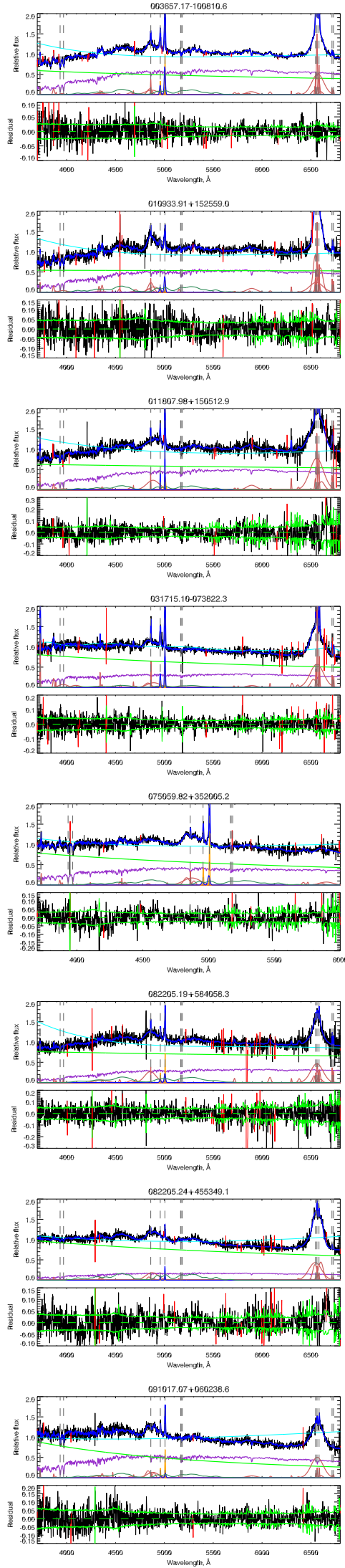
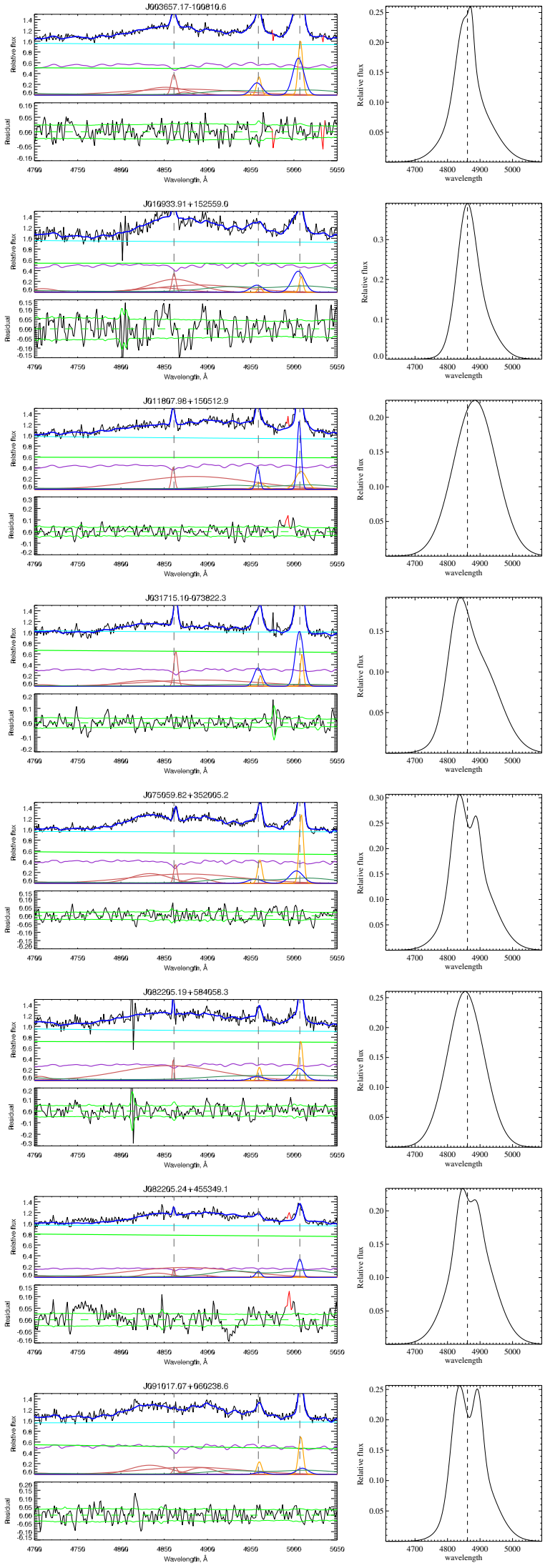

Fig. A.1. ULySS fits to the HG sample. In the upper left panel, the black line represents the observed spectrum, the blue one represents the best fit, the red line represents the multiplicative polynomial, while the green, light red, and violet lines represent components of the best fit: violet is the stellar population, red is the emission lines, and green is the AGN continuum. Bottom left panel: residuals of the best fit (black line). The green solid line shows the level of the noise, and the dashed line is the zero-axis. Middle panels zoom the domain around $\mathrm{H} \beta$ and [OIII] $\lambda 5007$ lines, while panels on the right hand side show the model broad $\mathrm{H} \beta$. 
A\&A 635, A151 (2020)

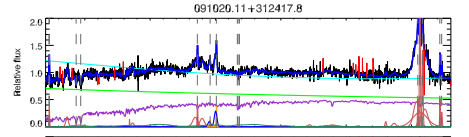

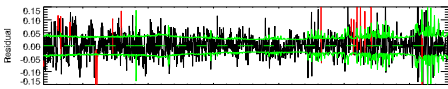
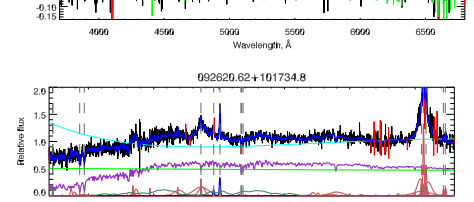

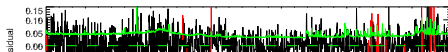

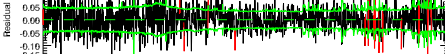

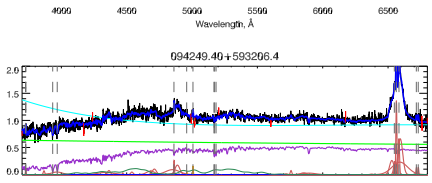

1.0.
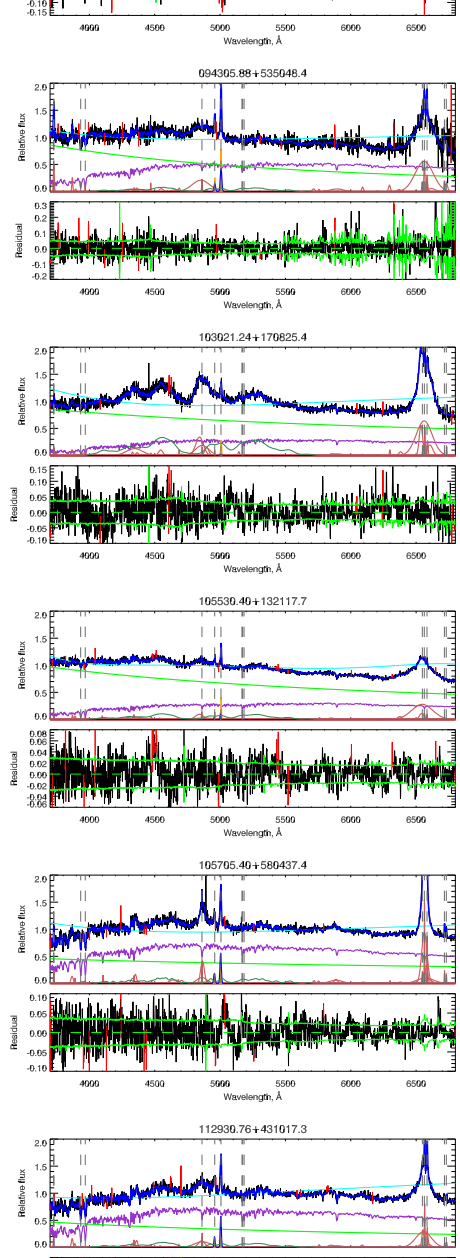

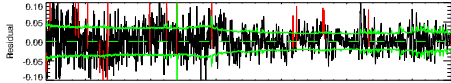
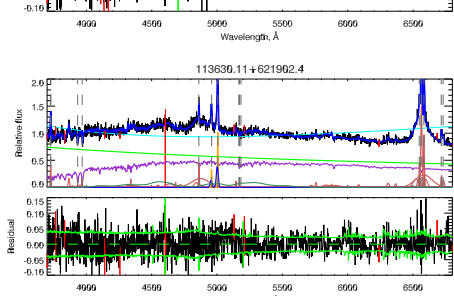
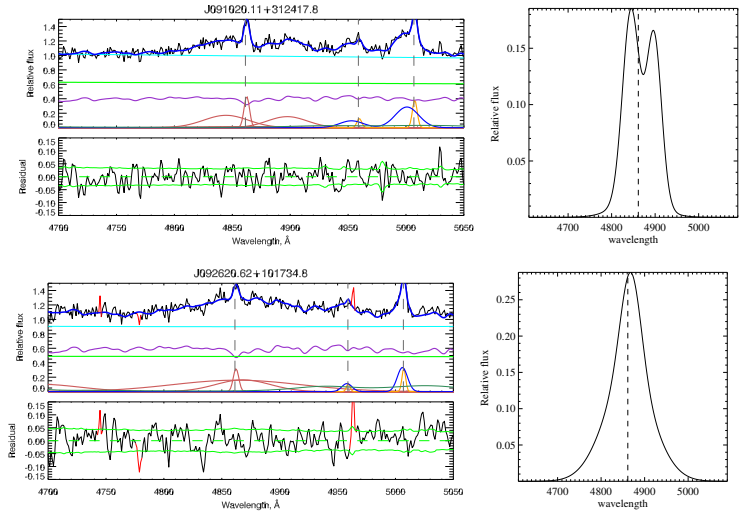

Jo94249.40+593206.4

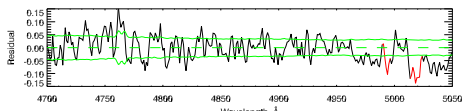
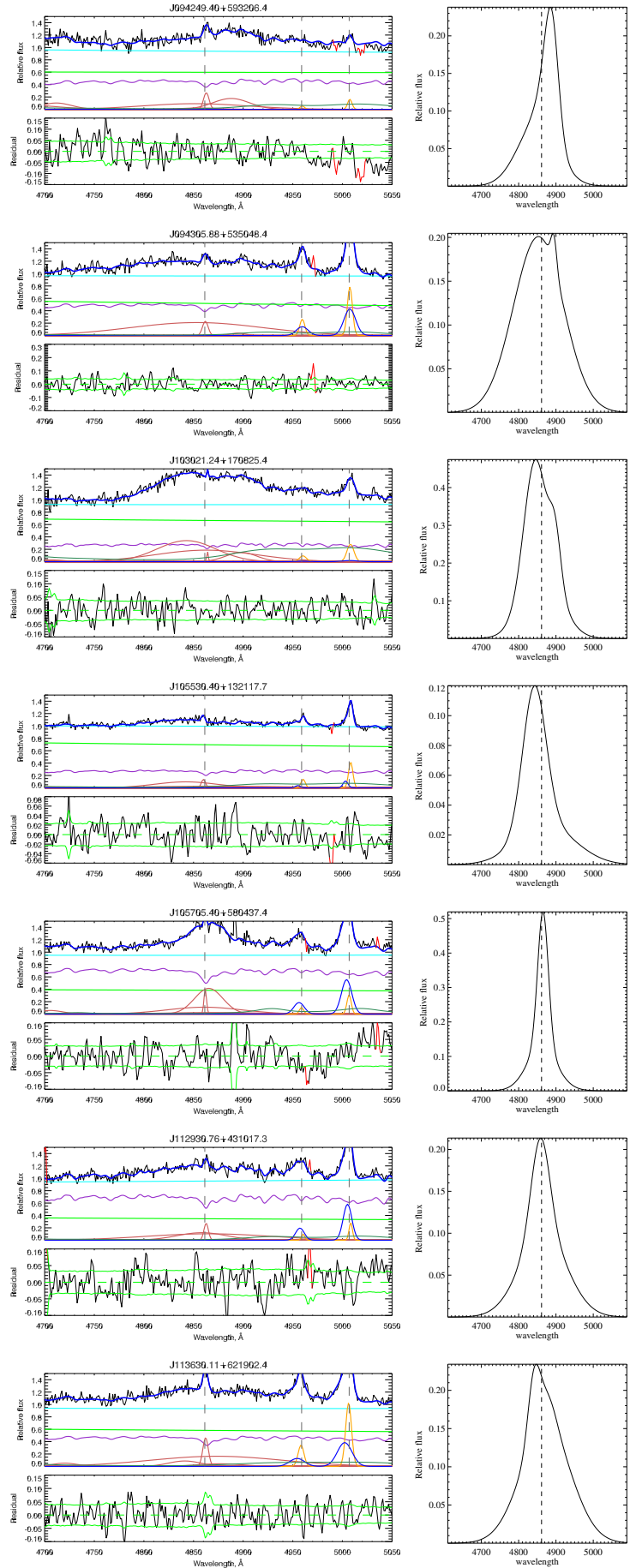

Fig. A.1. continued. 
N. Bon et al.: Narrow emission and absorption lines among Fe $\mathrm{II}_{\mathrm{opt}}$ strong low- $z$ quasars
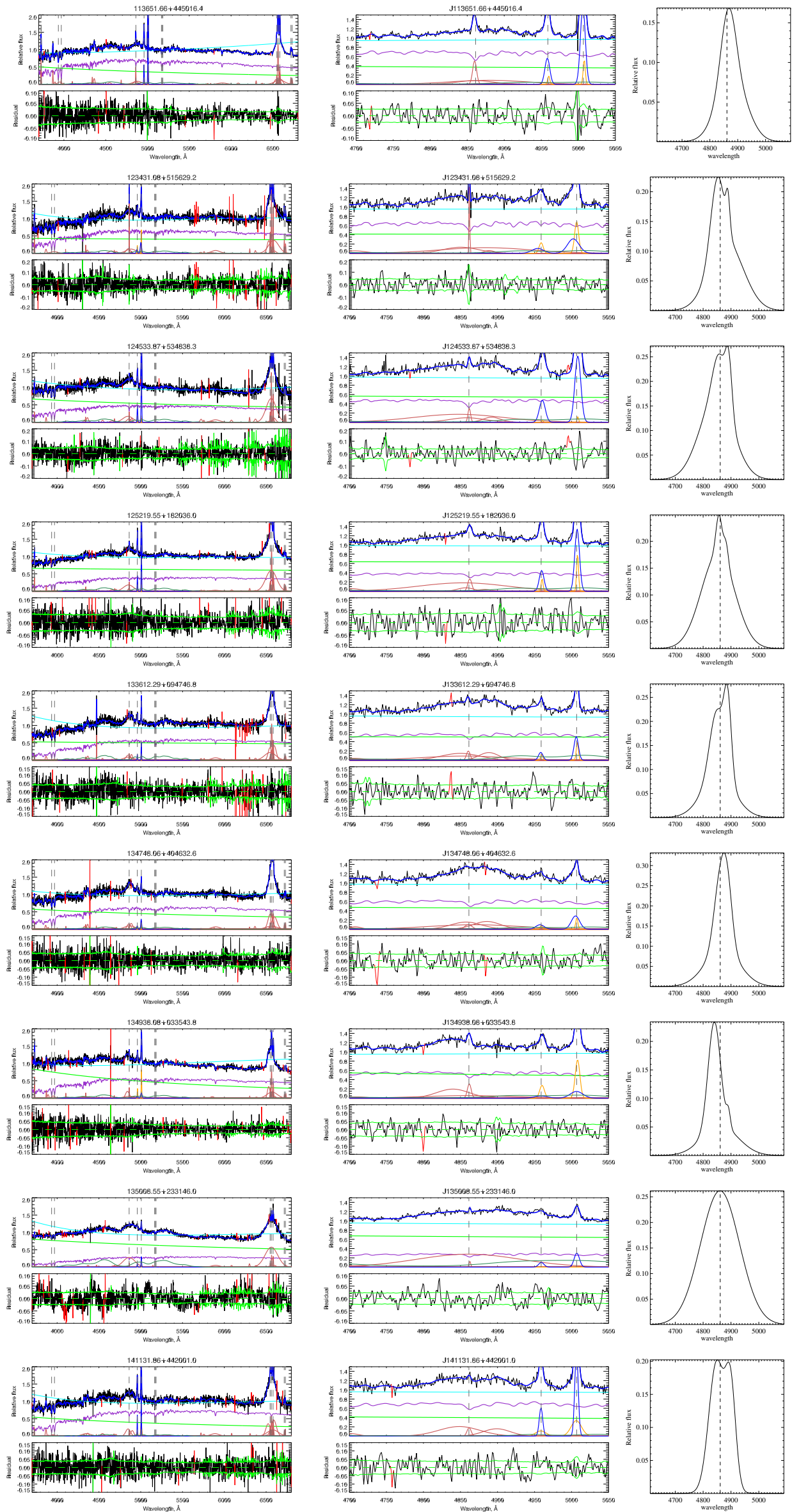

Fig. A.1. continued. 

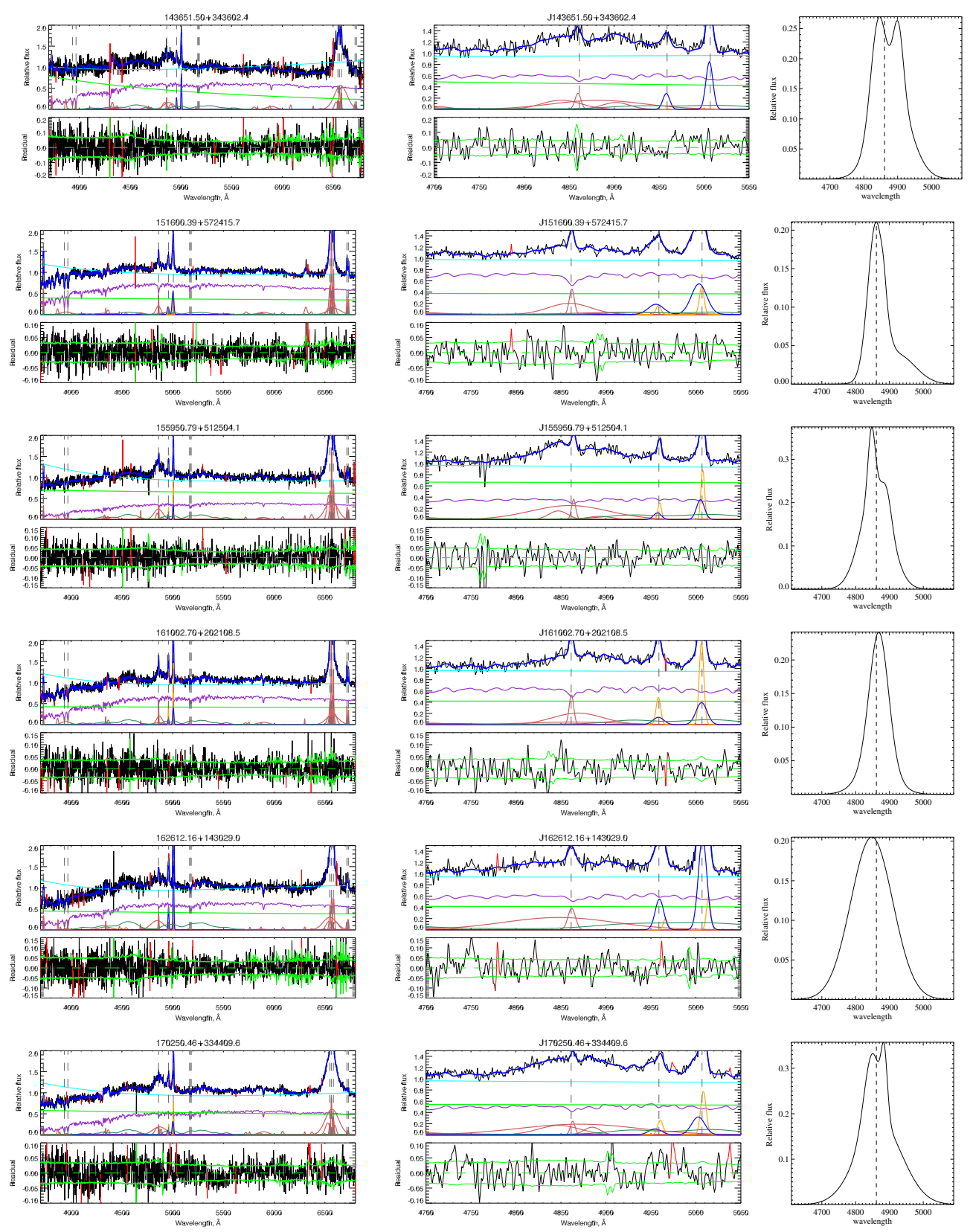

Fig. A.1. continued. 
N. Bon et al.: Narrow emission and absorption lines among Fe $\mathrm{II}_{\mathrm{opt}}$ strong low- $z$ quasars
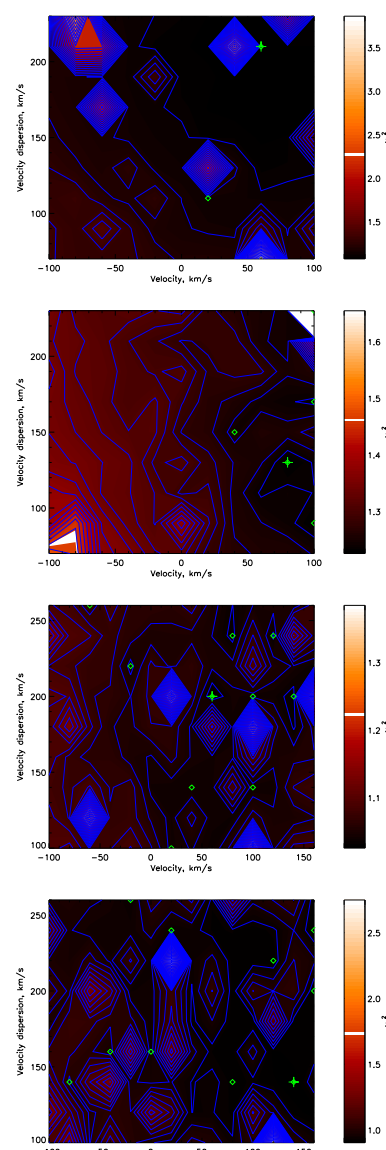

Vecocity, $.50 \%$

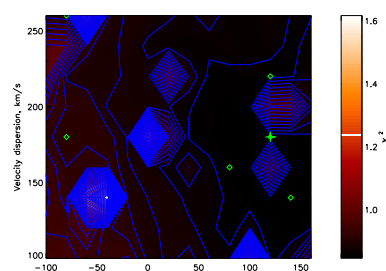

Velocity, $4.5 \%$

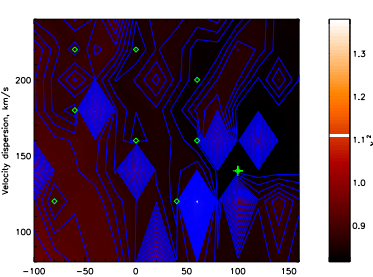

Velocity, $4 m / s$

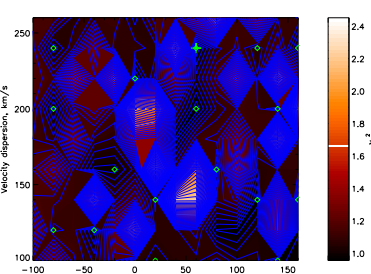

velocity, mint

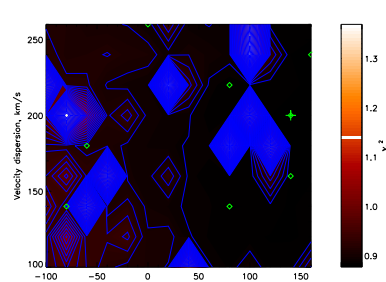

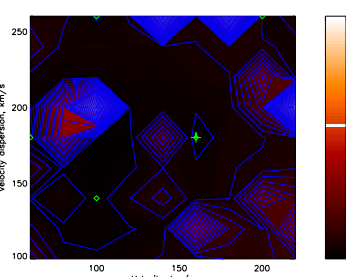
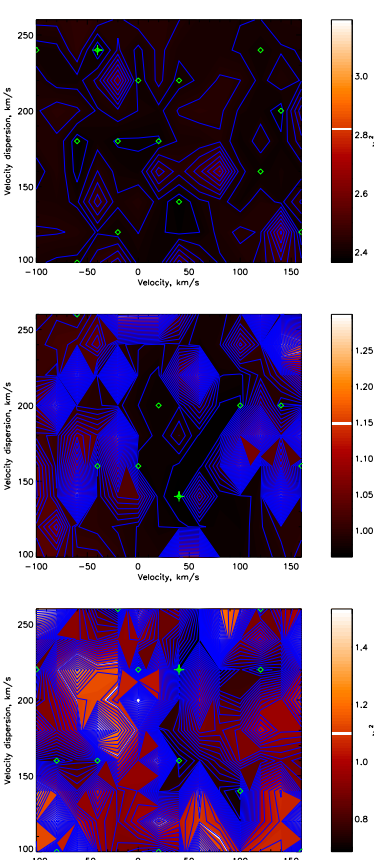

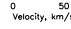
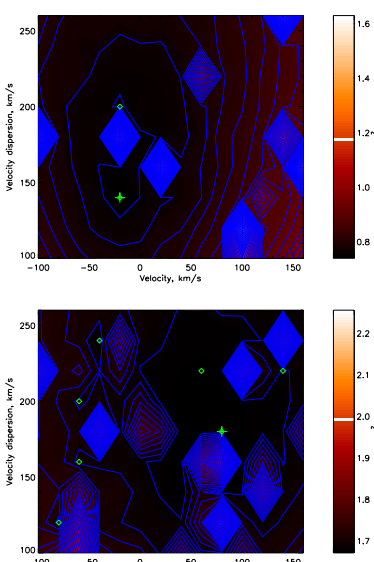

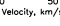
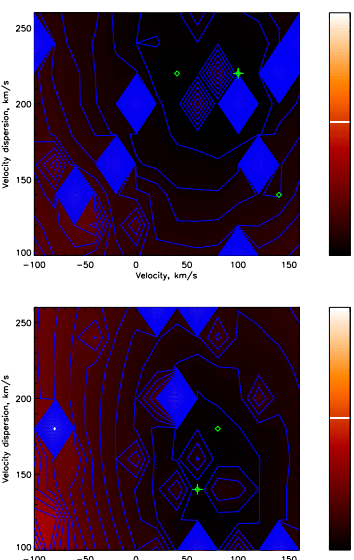
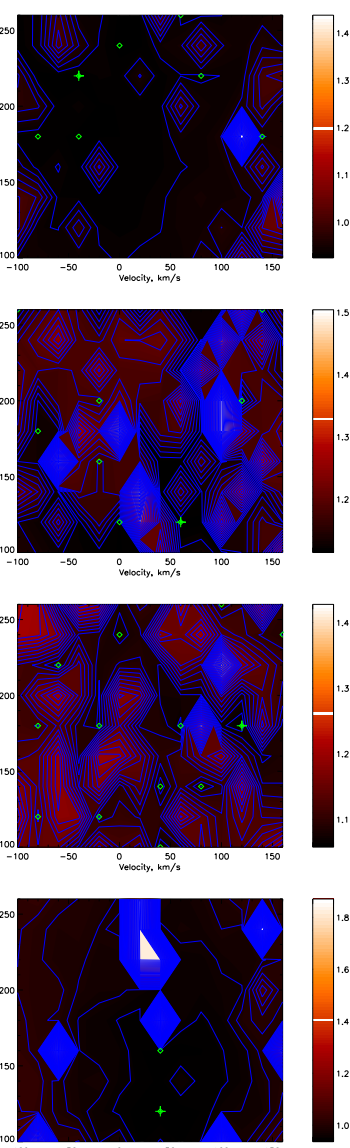

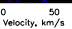
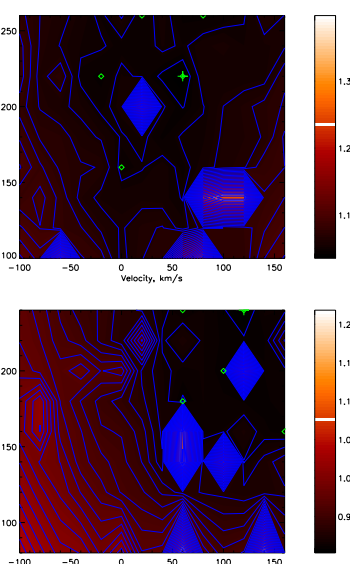

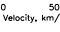
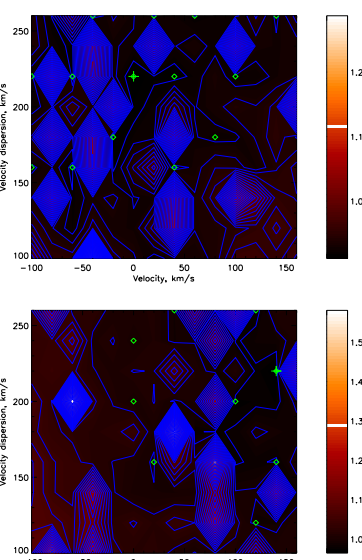
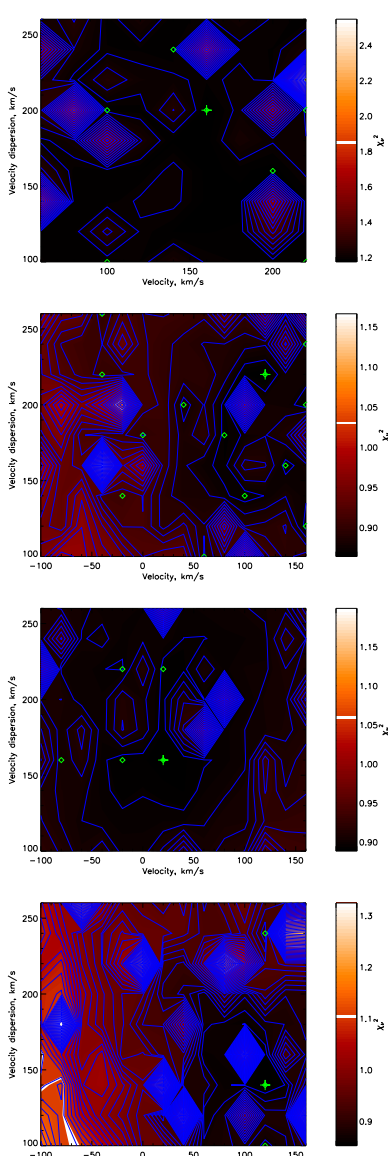

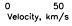
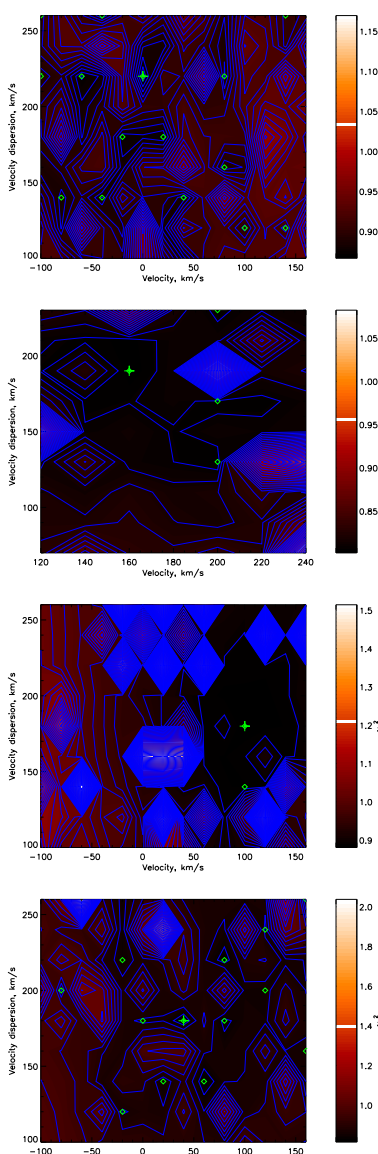

Fig. A.2. $\chi^{2}$ maps in the space of SSP mean stellar velocity and SSP velocity dispersion. Color-bar on the right hand side of $\chi^{2}$ maps show $\chi^{2}$ value normalized for the $\mathrm{S} / \mathrm{N}$ value of the spectrum, which was measured by the SDSS in the $g$-band. 


\section{Appendix B: A method to derive emissivity weighted $n_{\mathrm{e}}$ from $\lambda_{\mathrm{eff}}$ of [OII] $\lambda 3727$}

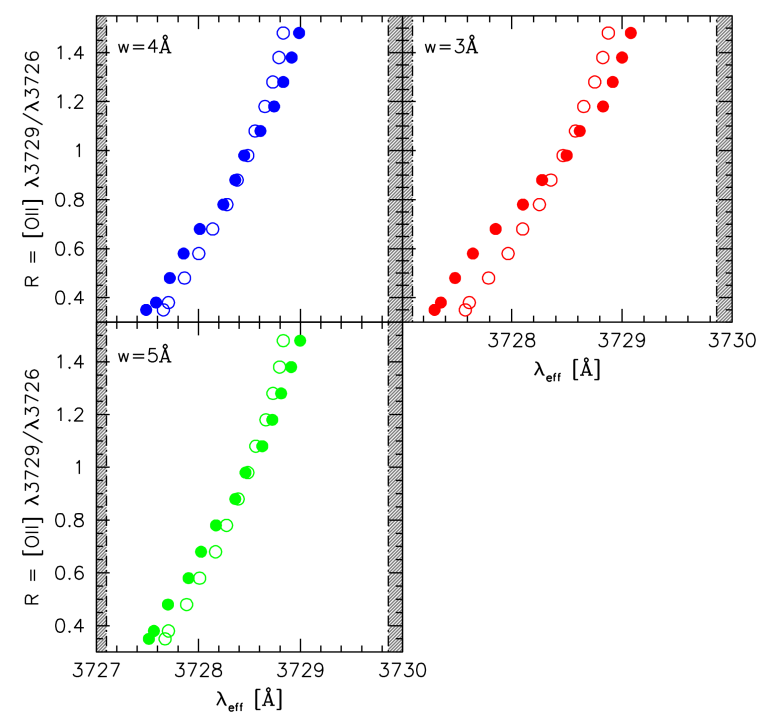

Fig. B.1. $\mathcal{R}[\mathrm{OII}]$ as a function of the $[\mathrm{OII}] \lambda \lambda 3726,3729$ doublet effective wavelength $\lambda_{\mathrm{eff}}$ for an unresolved mock doublet of FWHM 3, 4 and $5 \AA$. In each panel the open circles refer to Gaussian fits from the line base, and filled circles refer to Gaussian fits from half peak intensity. The vertical dot-dashed lines mark the position of the individual component of the [OII] doublet.

Figure B.1 shows the behavior of $\mathcal{R}=I([\mathrm{OII}] \lambda 3729) / I([\mathrm{OII}]$ 13726) as a function of the [OII] $\lambda \lambda 3726,3729$ doublet effective wavelength $\left(\lambda_{\text {eff }}\right)$ for an unresolved mock doublet of 4 and $5 \AA$. The $\lambda_{\text {eff }}$ has been measured for mock profiles built for 11 values of $R$ with a step $\delta \mathcal{R}=0.1$. A cubic or quadratic fit reproduces the value of $R$ :

$\mathcal{R}\left(n_{\mathrm{e}}\right)=k_{3}\left(\lambda_{\mathrm{eff}}-3727\right)^{3}+k_{2}\left(\lambda_{\mathrm{eff}}-3727\right)^{2}+k_{1}\left(\lambda_{\mathrm{eff}}-3727\right)+k_{0}$

with the coefficients given in Table B.1. The three width cases suggest a monotonic behavior of $\mathcal{R}$ as a function of $\lambda_{\text {eff }}$, with $\lambda_{\text {eff }}$ changing by $1.5 \AA$ from $3727.5 \AA$ and $3729.0 \AA$. The measurements carried out from half peak intensity (filled circles in Fig. B.1) are more sensitive to the centroid differences and should be preferred in practice to fits from the base of the line.

The best-fit parameters of $\mathcal{R}$ as a function of $n_{\mathrm{e}}$ using up-todate atomic data are shown in Table B.2 for [OII] from Sanders et al. (2016). Coefficients refer to a function of the form

$\mathcal{R}\left(n_{\mathrm{e}}\right)=a \frac{b+n_{\mathrm{e}}}{c+n_{\mathrm{e}}}$.

So the $\lambda_{\text {eff }}$ of [OII] can be related to the density as follows:

$\mathcal{R}\left(n_{\mathrm{e}}\right)=k_{2} \lambda_{\mathrm{eff}}^{2}+k_{1} \lambda_{\mathrm{eff}}+k_{0}=a \frac{b+n_{\mathrm{e}}}{c+n_{\mathrm{e}}}$.

Making this relation explicit for density:

$n_{\mathrm{e}}(\mathcal{R})=\frac{c \mathcal{R}-a b}{a-\mathcal{R}}$

$n_{\mathrm{e}}\left(\lambda_{\mathrm{eff}}\right)=\frac{c\left(k_{2} \lambda_{\mathrm{eff}}^{2}+k_{1} \lambda_{\mathrm{eff}}+k_{0}\right)-a b}{a-\left(k_{2} \lambda_{\mathrm{eff}}^{2}+k_{1} \lambda_{\mathrm{eff}}+k_{0}\right)}$.

An application of the method to the sample of the HII region from the SDSS DR1 by Kniazev et al. (2004) is shown
Table B.1. Coefficients in Eq. (B.1).

\begin{tabular}{cccccc}
\hline \hline$w$ & Fit & $k_{3}$ & $k_{2}$ & $k_{1}$ & $k_{0}$ \\
\hline 3 & Half & 0.241 & -0.7672 & +1.2428 & +0.0302 \\
3 & Full & 0 & 0.3353 & +0.0105 & +0.2473 \\
4 & Half & 0 & 0.1831 & +0.2726 & +0.1818 \\
4 & Full & 0 & 0.4356 & -0.1684 & +0.2879 \\
5 & Half & 0 & 0.1713 & +0.3065 & +0.1628 \\
5 & Full & 0 & 0.4492 & -0.2008 & +0.2982 \\
\hline
\end{tabular}

Table B.2. Coefficients and limiting line ratios for [OII] in Eq. (B.2)

\begin{tabular}{lccccc}
\hline \hline $\mathcal{R}$ & $a$ & $b$ & $c$ & $\mathcal{R}_{\min }{ }^{(a)}$ & $\mathcal{R}_{\max }{ }^{(b)}$ \\
\hline$[\mathrm{OII}] \lambda 3729 / \lambda 3726$ & 0.3771 & 2468 & 638.4 & 0.3839 & 1.4558 \\
\hline
\end{tabular}

Notes. ${ }^{(a)}$ Theoretical minimum line ratio calculated in the high-density limit of $100000 \mathrm{~cm}^{-3}$. ${ }^{(b)}$ Theoretical maximum line ratio calculated in the low-density limit of $1 \mathrm{~cm}^{-3}$.

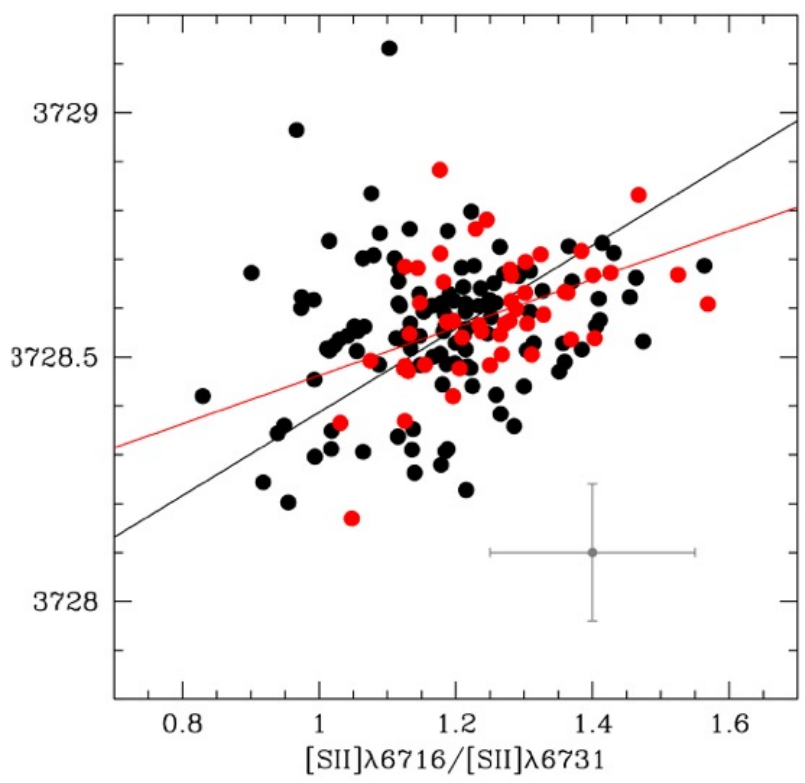

Fig. B.2. Relation between the [OII] $\lambda 3727$ effective wavelength $\lambda_{\text {eff }}$ and the $R[\mathrm{SII}]$ intensity ratio for a sample of HII regions. Red circles refer to a vetted subsample in which the uncertainty in the [SII] $\lambda \lambda 6731,6717$ doublet ratio $R$ is less than $10 \%$. Best fitting lines are obtained with the bisector method (full sample, black line) and with an unweighted least square fit (vetted sample, blue line). Typical errors are $\delta \lambda_{\mathrm{eff}} \approx \pm 0.15 \AA$ for the effective wavelength and $\delta R[\mathrm{SII}] \approx \pm 0.15$ for the $[\mathrm{SII}] \lambda \lambda 6731,6717$ ratio.

in Fig. B.2. The $\lambda_{\text {eff }}$ and $R[\mathrm{SII}]$ are correlated. The scatter is relatively large. Typical errors $(\delta R[\mathrm{SII}] \approx 0.15)$ suggest that measurement uncertainties account for most or all of it. If a restriction of $\delta R[\mathrm{SII}] \lesssim 0.10$ is applied, the correlation is better defined, with a Pearson correlation coefficient $r \approx 0.46$, implying a significance $\gtrsim 4 \sigma$. The average value of $R$ [SII] and $\lambda_{\text {eff }}$ [OII] $\lambda 3727$ are 1.21 and $3728.56 \AA$, respectively. The $R$ [SII] value implies $n_{\mathrm{e}} \approx 10^{2.4} \mathrm{~cm}^{-3} ; \lambda_{\text {eff }}[\mathrm{OII}] \lambda 3727$ implies $\mathcal{R}[\mathrm{OII}] \approx 1.1$, which in turn yields $n_{\mathrm{e}} \approx 10^{2.5} \mathrm{~cm}^{-3}$. The estimators are therefore consistent on average. This result indicates that, especially in some ideal cases, the $\lambda_{\text {eff }}$ can be considered as a proxy of the [OII] $\lambda 3727$ doublet component ratio $\mathcal{R}$ and hence it is an appropriate $n_{\mathrm{e}}$ estimator. 